\title{
Coinductive predicates and final sequences in a fibration
}

\author{
ICHIRO $\mathrm{HASUO}^{\dagger}$, TOSHIKI KATAOKA ${ }^{\dagger \dagger}$ and KENTA $\mathrm{KHO}^{\S}$ \\ ${ }^{\dagger}$ Department of Computer Science, The University of Tokyo, Tokyo 113-8656, Japan \\ Email: ichiro@is.s.u-tokyo.ac.jp \\ ${ }^{\ddagger}$ Research Fellow of Japan Society for the Promotion of Science, 5-3-1, Kouji-machi, \\ Chiyoda-ku, Tokyo 102-0083, Japan \\ Email: toshikik@is.s.u-tokyo.ac.jp \\ $\S$ Institute for Computing and Information Sciences, Radboud University, \\ P.O.Box 9010, 6500 GL Nijmegen, the Netherlands \\ Email: K.Cho@cs.ru.nl
}

Received 9 January 2015; revised 22 November 2016

Coinductive predicates express persisting 'safety' specifications of transition systems.

Previous observations by Hermida and Jacobs identify coinductive predicates as suitable final coalgebras in a fibration - a categorical abstraction of predicate logic. In this paper, we follow the spirit of a seminal work by Worrell and study final sequences in a fibration. Our main contribution is to identify some categorical 'size restriction' axioms that guarantee stabilization of final sequences after $\omega$ steps. In its course, we develop a relevant categorical infrastructure that relates fibrations and locally presentable categories, a combination that does not seem to be studied a lot. The genericity of our fibrational framework can be exploited for binary relations (i.e. the logic of 'binary predicates') for which a coinductive predicate is bisimilarity, constructive logics (where interests are growing in coinductive predicates) and logics for name-passing processes.

\section{Introduction}

Coinductive predicates postulate properties of state-based dynamic systems that persist after a succession of transitions. In computer science, safety properties of non-terminating, reactive systems are examples of paramount importance. This has led to an extensive study of specification languages in the form of fixed point logics and model-checking algorithms.

In this paper, we follow Hermida and Jacobs (1998) and Hermida (1993) - whose results are further extended in Fumex et al. (2011) and Atkey et al. (2012), see also Jacobs (2012, Chap. 6) - and take a categorical view on coinductive predicates. Here, coalgebras represent transition systems; a fibration is a 'predicate logic'; and a coinductive predicate is identified as a suitable coalgebra in a fibration. Our contribution is the study of final sequences - an iterative construction of final coalgebras that is studied notably in Worrell (2005) and Adámek (2003) - in such a fibrational setting.

Coalgebras have been successfully used as a categorical abstraction of transition systems (see e.g. Jacobs (2012); Rutten (2000)): By varying base categories and functors, coalgebras bring general results that work for a variety of systems at once. Fixed point logics (or modal logics in general), too, have been actively studied coalgebraically: Coalgebraic 
modal logic is a prolific research field (see Cîrstea et al. 2011); their base category is typically Sets but works like Klin (2007) go beyond and use presheaf categories for processes in name-passing calculi; and literature including Cîrstea and Sadrzadeh (2008), Venema (2006) and Cîrstea et al. (2009) studies coalgebraic fixed point logics.

Unlike most of these works, we follow Hermida and Jacobs (1998) and Hermida (1993) and parameterize the underlying 'predicate logic' too with the categorical notion of fibration. The conventional setting of classical logic is represented by the fibration $\begin{gathered}\text { Pred } \\ \downarrow\end{gathered}$ (see Appendix $\mathrm{C}$ for an introduction to fibrations).

\begin{tabular}{|c|c|c|}
\hline fibration $\underset{\mathbb{C}}{\stackrel{\mathbb{P}}{\downarrow}}$ & $\begin{array}{c}\text { Pred } \\
\downarrow \\
\text { Sets }\end{array}$ & $\begin{array}{c}\text { Rel } \\
\downarrow \\
\text { Sets }\end{array}$ \\
\hline coalgebra & invariant & bisimulation \\
\hline $\begin{array}{c}\text { final } \\
\text { coalgebra }\end{array}$ & $\begin{array}{l}\text { coinductive } \\
\text { predicate }\end{array}$ & bisimilarity \\
\hline
\end{tabular}

However, there are various other 'logics' modelled as fibrations, and hence the fibrational language provides a uniform treatment of these different settings. An example is binary relations (instead of unary predicates) that form a fibration $\underset{\text { Sels }}{\downarrow}$ (see Appendix C). In this case, coinductive predicates are bisimilarity relations (see the above table, and Example 7.2 later).

Another example is predicates in constructive logics. They are modelled by the subobject fibration of a topos. In fact, coinductive predicates in constructive logics are an emerging research topic: Coinduction is supported in the theorem prover Coq (based on the constructive calculus of constructions), see e.g. Bertot and Komendantskaya (2008), and working in Coq, some interesting differences between classically equivalent (co)inductive predicates have been studied, e.g. in Nakata et al. (2011).

Yet another example is modal logics for processes in various name-passing calculi. They are best modelled by the subobject fibration of a suitable (pre)sheaf category like Sets ${ }^{\mathbf{I}}$ and Sets $^{\mathbf{F}}$ (Fiore and Turi 2001; Fiore and Staton 2006; Miculan 2008; Stark 1996; Staton 2011).

\subsection{Coinductive predicates and their construction, conventionally}

In order to illustrate our technical contributions (Section 3), we here present a special case, with classical logic and Kripke models. We first introduce syntax.

Definition 1.1 (Rudimentary logic $\mathrm{R} v$ ). In this tiny fragment of the $\mu$-calculus, fixed-point operators are limited to the greatest one at the outermost position; and moreover, all the formulas are 'rank-1,' that is, the fixed-point variable $u$ occurs precisely under one modal operator.

$$
\mathrm{R} v_{u} \ni \alpha::=a|\bar{a}| \square u|\diamond u| \alpha \wedge \alpha \mid \alpha \vee \alpha ; \quad \operatorname{R} v \ni \beta::=v u . \alpha .
$$

Here, $a$ belongs to the set AP of atomic propositions; $\bar{a}$ stands for the negation of $a$ and $u$ is the only fixed-point variable (with possibly multiple occurrences). 
An $R v$-formula can be thought of as a recursive definition of a coinductive predicate. Later, we will model such a 'definition' categorically as a predicate lifting. Among specifications expressible in $\mathrm{R} v$ is (may-) deadlock freedom (there is an infinite path). It is expressed by $v u$. $\diamond u$ and is our recurring example.

An $\mathrm{R} v$-formula is interpreted in Kripke models. Let $c=(X, \rightarrow, V)$ be a Kripke model, where $X$ is a state space, $\rightarrow \subseteq X \times X$ is a transition relation and $V: X \rightarrow \mathcal{P}(\mathrm{AP})$ is a valuation. The conventional interpretation $[v u . \alpha]_{c}$ of $\mathrm{R} v$-formulas in the Kripke model $c$ is given as follows (see e.g. Bradfield and Stirling (2006)). First, we interpret $\alpha \in R v_{u}$ as a function $[\alpha]_{c}: \mathcal{P} X \rightarrow \mathcal{P} X$. Concretely:

$$
\begin{aligned}
{[a]_{c}(P) } & =\{x \mid a \in V(x)\} & {[\bar{a}]_{c}(P) } & =\{x \mid a \notin V(x)\} \\
{[\square u]_{c}(P) } & =\{x \mid \forall y \in X .(x \rightarrow y \text { implies } y \in P)\} & & {\left[\alpha \wedge \alpha^{\prime}\right]_{c}(P)=[\alpha]_{c}(P) \cap\left[\alpha^{\prime}\right]_{c}(P) } \\
{[\diamond u]_{c}(P) } & =\{x \mid \exists y \in X .(x \rightarrow y \text { and } y \in P)\} & & {\left[\alpha \vee \alpha^{\prime}\right]_{c}(P)=[\alpha]_{c}(P) \cup\left[\alpha^{\prime}\right]_{c}(P) }
\end{aligned}
$$

This function $[\alpha]_{c}$ is easily seen to be monotone, since $u$ occurs only positively in $\alpha$. Finally, we define $[v u . \alpha]_{c} \subseteq X$ to be the greatest fixed point of the monotone function $[\alpha]_{c}: \mathcal{P} X \rightarrow \mathcal{P} X$.

The Knaster-Tarski theorem guarantees the existence of such a greatest fixed point $[v u . \alpha]_{c}$ in a complete lattice $\mathcal{P} X$. However, its proof is highly non-constructive. In contrast, a well-known iterative construction (Cousot and Cousot 1979) computes $[v u . \alpha]_{c}$ as the limit of the following descending chain (see also Bradfield and Stirling (2006)). Here, $\top$ denotes the subset $X \subseteq X$.

$$
\top \geqslant[\alpha]_{c} \top \geqslant[\alpha]_{c}^{2} \top \geqslant \cdots
$$

An issue now is the length of the chain. If $[\alpha]_{c}$ preserves limits $\wedge$ (which is the case with $\alpha \equiv \square u)$, clearly $\omega$ steps are enough and yields $\bigwedge_{i \in \omega}\left([\alpha]_{c}^{i} \top\right)$ as the greatest fixed point. This is not the case with $\alpha \equiv \diamond u$. Indeed, for the Kripke model $c_{1}$ below $[v u . \diamond u]_{c_{1}} \neq \bigwedge_{i \in \omega}\left([\diamond u]_{c_{1}}^{i} \top\right):$ there is no infinite path from the root; but it satisfies $[\diamond u]_{c_{1}}^{i} \top$ (there is a path of length $\geqslant i$ ) for each $i$.

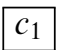

Yet the chain (2) eventually stabilizes, bounded by the size of the poset $\mathcal{P} X$ : In each step before stabilization, at least one element must be thrown away. Therefore, the calculation of $[v u . \alpha]_{c}$ proceeds, in general, via transfinite induction. This is what we call a state space bound for the chain (2).

Besides a state space bound, another (possibly better and seemingly less known) bound can be obtained from a behavioural view. One realizes that not only the size of the state space $X$ but also the branching degree can be used to bound the length of the chain (2). 
This is a result similar to the one in Hennessy and Milner (1985, Theorem 2.1); the latter is stated for bisimilarity as a coinductive relation, not for a coinductive predicate. We formally state (an instance of) the result for the record.

Lemma 1.2 (Behavioural bound). Let $c=(X, \rightarrow, V)$ be a finitely branching Kripke model. For $\alpha=\diamond u$, the chain (2) stabilizes after $\omega$ steps and yields $[v u . \diamond u]_{c}$ as its limit, that is, $\bigwedge_{i \in \omega}\left([\diamond u]_{c}^{i} \top\right)=[v u . \diamond u]_{c}$.

Proof. The essence of the result lies in the fact that the limit $\bigwedge_{i \in \omega}\left([\diamond u]_{c}^{i} \top\right)$ is a $\diamond$ invariant, which we shall prove now. Assume that a state $x$ satisfies $\bigwedge_{i \in \omega}\left([\diamond u]_{c}^{i \top}\right)$; we have to show that $x$ satisfies $[\diamond u]_{c}\left(\bigwedge_{i \in \omega}\left([\diamond u]_{c}^{i} \top\right)\right)$, that is, there is a successor $x^{\prime}$ of $x$ that satisfies the limit $\bigwedge_{i \in \omega}\left([\diamond u]_{c}^{i} \top\right)$.

Since $x$ satisfies $[\diamond u]_{c}^{i} \top$ (there is a path of length $\geqslant i$ ) for each $i$, for each $i \geqslant 1$, there is a successor $x_{i}$ of $x$ that satisfies $[\diamond u]_{c}^{i-1} T$. By $c$ being finitely branching, the set $\left\{x_{1}, x_{2}, \ldots\right\}$ of such successors turns out to be finite and there exists a successor $x^{\prime}$ of $x$ such that $x^{\prime}=x_{i}$ for infinitely many $i$. It follows (from $[\diamond u]_{c}^{i} \top \leqslant[\diamond u]_{c}^{j} \top$ if $j \leqslant i$ ) that this $x^{\prime}$ satisfies $[\diamond u]_{c}^{i} \top$ for all $i \in \omega$, and hence satisfies $\bigwedge_{i \in \omega}\left([\diamond u]_{c}^{i} \top\right)$. This proves that the limit $\bigwedge_{i \in \omega}\left([\diamond u]_{c}^{i} \top\right)$ is an invariant, and hence $\bigwedge_{i \in \omega}\left([\diamond u]_{c}^{i} \top\right) \leqslant[v u . \diamond u]_{c}$.

For the last equality claimed in the lemma, the other direction $[v u . \diamond u]_{c} \leqslant \bigwedge_{i \in \omega}\left([\diamond u]_{c}^{i} \top\right)$ is easy: $[v u . \diamond u]_{c} \leqslant[\diamond u]_{c}^{i} \top$ is easily shown by induction on $i$. This concludes the proof.

Note that Lemma 1.2 holds however large the state space $X$ is. Moreover, it easily generalizes from $v u . \diamond u$ to an arbitrary $\mathrm{R} v$-formula $v u . \alpha$. Note also that the counterexample $c_{1}$ in Equation (3) is not finitely branching and does not contradict with Lemma 1.2.

\subsection{Final sequences in a fibration}

This paper is about putting the observations in Section 1.1 in general categorical terms. Our starting observation is that the chain (2) resembles a final sequence, a classic construction of a final coalgebra.

In the theory of coalgebra, a final F-coalgebra is of prominent importance since it is a fully abstract domain with respect to the $F$-behavioural equivalence. Therefore, a natural question is if a final $F$-coalgebra exists; the well-known Lambek lemma prohibits e.g. a final $\mathcal{P}$-coalgebra for the (full) powerset functor $\mathcal{P}$. What matters is the size of $F$ : When it is suitably bounded, it is known that a final coalgebra can be constructed via the following final F-sequence:

$$
1 \longleftarrow \text { ! } F 1 \stackrel{F !}{\longleftarrow} \cdots \stackrel{F^{i-1} !}{\longleftarrow} F^{i} 1 \stackrel{F^{i} !}{\longleftarrow} \cdots
$$

Here, 1 is a final object in $\mathbb{C}$, and ! is the unique arrow. In particular, if $F$ is finitary, a final coalgebra arises as a suitable subobject (or a quotient) of the $\omega$-limit of the final sequence (4). These constructions in Sets are worked out in Pattinson (2003) and Worrell (2005); the one in Worrell (2005) is further extended to locally presentable categories (those are categories suited for speaking of 'size') with additional assumptions in Adámek (2003). 
Turning back to coinductive predicates, indeed, the fibrational view (Hermida 1993; Hermida and Jacobs 1998) identifies coinductive predicates as final coalgebras in a fibration. This leads us to scrutinize final sequences in a fibration. Our main result (Theorem 3.9) is a categorical generalization of the behavioural $\omega$-bound (Section 1.1) - more precisely, we axiomatize categorical 'size restrictions' for that bound to hold.

The conditions are formulated in the language of locally presentable categories (see e.g. Adámek and Rosický (1994); also Appendix B); and the combination of fibrations and locally presentable categories does not seem to have been studied a lot (an exception is Makkai and Paré (1989, Section5.3)). We therefore develop a relevant categorical infrastructure (Section 6). Our results there include a sufficient condition for the total category $\operatorname{Sub}(\mathbb{C})$ of a subobject fibration to be locally (finitely) presentable, and the same for a family fibration $\operatorname{Fam}(\Omega)$. Via these results, in Section 7, we list some concrete examples of fibrations to which our results in Section 3 on the behavioural bounds apply. They include $\underset{\text { Sets }}{\downarrow}$ (classical logic), $\underset{\text { Sets }}{\downarrow}$ (for bisimulation and bisimilarity), $\stackrel{\operatorname{Sub}(\mathbb{C})}{\downarrow}$ for $\mathbb{C}$ that is locally finitely presentable (LFP) and locally Cartesian closed (a topos is a special case) and $\underset{\text { Sets }}{\stackrel{\operatorname{Fam}(\Omega)}{\downarrow}}$ for a well-founded algebraic lattice $\Omega$.

\subsection{Contributions}

To summarize, our contributions are (1) combination of the mathematical observations in Hermida (1993), Hermida and Jacobs (1998) and (Jacobs 2012, Chap. 6) for a general formulation of coinductive predicates; (2) categorical behavioural bounds for final sequences that approximate coinductive predicates and (3) a categorical infrastructure that relates fibrations and locally presentable categories.

Compared to the earlier version (Hasuo et al. 2013) of the current paper, the main differences are as follows. Here, we additionally address inductive predicates over coinductive datatypes (see Section 5). We identify them as coinductive predicates in the fibrewise

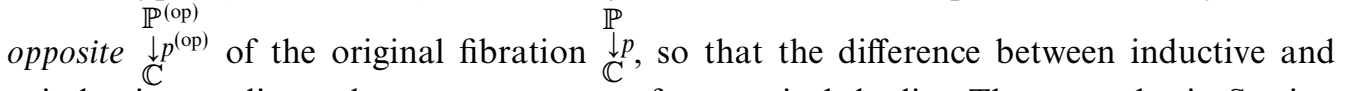
coinductive predicates becomes a matter of categorical duality. The examples in Section 7 are extended accordingly, studying inductive predicates on top of coinductive ones. Besides, we include all the proofs that were omitted in Hasuo et al. (2013) for space reasons.

\subsection{Organization of the paper}

In Section 2, we identify coinductive predicates as final coalgebras in a fibration, following the ideas of Hermida (1993), Hermida and Jacobs (1998) and Jacobs (2012). The main technical results are in Section 3, where we axiomatize size restrictions on fibrations and functors for a final sequence to stabilize after $\omega$ steps. These results are reorganized in Section 4 in a fibration of invariants. We see in Section 5, which is added to an earlier version of this paper (Hasuo et al. 2013), that the results in Section 2-4 apply to inductive 
predicates too. The next two sections are devoted to examples: First in Section 6, we develop a necessary categorical infrastructure; and then in Section 7, we discuss concrete examples. In Section 8, we conclude with some directions of future work. In Appendices, we present minimal introductions to the theories of coalgebras, locally presentable categories and fibrations - the three categorical disciplines that our technical developments rely on.

\section{Coinductive predicates as final coalgebras}

In this section, we follow the ideas in Hermida (1993), Hermida and Jacobs (1998), Jacobs (2012) and characterize coinductive predicates in various settings (for different behaviour types, and in various underlying logics) in the language of fibrations. An introduction to fibrations is e.g. in Jacobs (1999); see also Appendix C. In this paper, for simplicity, we focus on poset fibrations. It should however not be hard to move to general fibrations.

Convention 2.1 (Fibration). We refer to poset fibrations (where each fibre is a poset rather than a category) simply as fibrations.

Definition 2.2 (Predicate lifting). Let $\underset{\mathbb{C}}{\mathbb{P}}$ be a fibration and $F$ be an endofunctor on $\mathbb{C}$. A predicate lifting of $F$ along $p$ is a functor $\varphi: \mathbb{P} \rightarrow \mathbb{P}$ such that $(\varphi, F)$ is an endomap of fibrations.

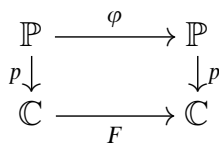

This means: that the above diagram commutes; and that $\varphi$ preserves Cartesian arrows, that is, $\varphi\left(f^{*} Q\right)=(F f)^{*}(\varphi Q)$. See below.

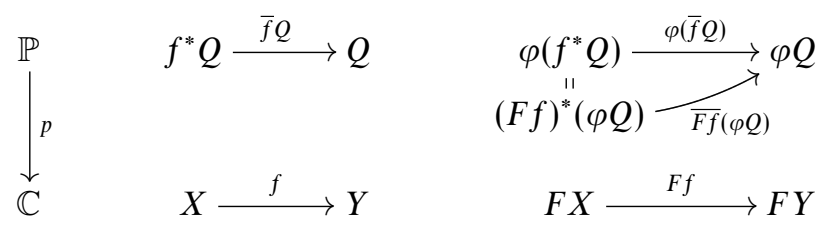

In the prototype example $\begin{gathered}\text { Pred } \\ \text { Sets }\end{gathered}$, the above definition coincides (see Jacobs 2012) with the one used in coalgebraic modal logic (see e.g. Cîrstea et al. (2011)), the latter being a (monotone) natural transformation $2^{(-)} \stackrel{\varphi}{\Rightarrow} 2^{F(-)}$ : Sets ${ }^{\text {op }} \rightarrow$ Sets. In particular, the naturality requirement corresponds to the preservation of Cartesian arrows (6); and monotonicity of $\varphi$ comes from the functoriality of $\varphi: \mathbb{P} \rightarrow \mathbb{P}$.

We think of predicate liftings as (co)recursive definitions of coinductive predicates (see Example 2.4). On top of it, we identify coinductive predicates (and invariants) as coalgebras in a fibre. 
Definition 2.3 (Invariant, coinductive predicate). Let $\varphi$ be a predicate lifting of $F$ along $\underset{\mathbb{C}}{\mathbb{P}}$; ; and $X \stackrel{c}{\rightarrow} F X$ be a coalgebra in $\mathbb{C}$. They together induce an endofunctor (a monotone function) on the fibre $\mathbb{P}_{X}$, namely $\mathbb{P}_{X} \stackrel{\varphi}{\rightarrow} \mathbb{P}_{F X} \stackrel{c^{*}}{\rightarrow} \mathbb{P}_{X}$, where $\varphi$ restricts to $\mathbb{P}_{X} \rightarrow \mathbb{P}_{F X}$ because of Equation (5).

1. A $\varphi$-invariant in $c$ is a $\left(c^{*} \circ \varphi\right)$-coalgebra in $\mathbb{P}_{X}$, that is, an object $P \in \mathbb{P}_{X}$ such that $P \leqslant c^{*}(\varphi P)$ in $\mathbb{P}_{X}$.

2. The $\varphi$-coinductive predicate in $c$ is the final $\left(c^{*} \circ \varphi\right)$-coalgebra (if it exists). Its carrier shall be denoted by $\llbracket v \varphi \rrbracket_{c}$. It is therefore the largest $\varphi$-invariant in $c$; Lambek's lemma yields that $\llbracket v \varphi \rrbracket_{c}=\left(c^{*} \circ \varphi\right)\left(\llbracket v \varphi \rrbracket_{c}\right)$.

Example 2.4 $(\mathrm{R} v)$. The conventional interpretation $[v u . \alpha]_{c}$ (described in Section 1.1) of $\mathrm{R} v$-formulas is a special case of Definition 2.3. Indeed, let us work in the fibration $\begin{gathered}\text { Pred } \\ \downarrow \\ \text { Sets }\end{gathered}$ and with the endofunctor $F_{\mathrm{K}}=\mathcal{P}(\mathrm{AP}) \times \mathcal{P}\left({ }_{-}\right)$on Sets. An $F_{\mathrm{K}}$-coalgebra $X \stackrel{c}{\rightarrow} \mathcal{P}(\mathrm{AP}) \times \mathcal{P} X$ is precisely a Kripke model: $c$ combines a valuation $X \rightarrow \mathcal{P}(\mathrm{AP})$ and the map $X \rightarrow \mathcal{P} X$ that carries a state to the set of its successors. To each formula $\alpha \in \mathrm{R} v_{u}$, we associate a predicate lifting $\varphi_{\alpha}$ of $F_{\mathrm{K}}$. This is done inductively as follows:

$$
\begin{aligned}
\varphi_{a}(U \subseteq X) & =\left(\left\{V \in F_{\mathrm{K}} X \mid a \in \pi_{1}(V)\right\} \subseteq F_{\mathrm{K}} X\right), \\
\varphi_{\bar{a}}(U \subseteq X) & =\left(\left\{V \in F_{\mathrm{K}} X \mid a \notin \pi_{1}(V)\right\} \subseteq F_{\mathrm{K}} X\right), \\
\varphi_{\square u}(U \subseteq X) & =\left(\left\{V \in F_{\mathrm{K}} X \mid \pi_{2}(V) \subseteq U\right\} \subseteq F_{\mathrm{K}} X\right), \\
\varphi_{\diamond u}(U \subseteq X) & =\left(\left\{V \in F_{\mathrm{K}} X \mid \pi_{2}(V) \cap U \neq \varnothing\right\} \subseteq F_{\mathrm{K}} X\right), \\
\varphi_{\alpha \wedge \alpha^{\prime}}(U \subseteq X) & =\left(\left(\varphi_{\alpha} U \cap \varphi_{\alpha^{\prime}} U\right) \subseteq F_{\mathrm{K}} X\right), \\
\varphi_{\alpha \vee \alpha^{\prime}}(U \subseteq X) & =\left(\left(\varphi_{\alpha} U \cup \varphi_{\alpha^{\prime}} U\right) \subseteq F_{\mathrm{K}} X\right) .
\end{aligned}
$$

In the above, $\pi_{1}$ and $\pi_{2}$ denote the projections from $F_{\mathrm{K}} X=\mathcal{P}(\mathrm{AP}) \times \mathcal{P} X$. Then it is easily seen by induction that $\llbracket v \varphi_{\alpha} \rrbracket_{c}$ in Definition 2.3 coincides with the conventional interpretation $[v u . \alpha]_{c}$ described in Section 1.1.

In fact, the predicate liftings $\varphi_{\alpha}$ in Equation (7) are the ones commonly used in coalgebraic modal logic (where they are presented as natural transformations). We point out that the same definition of $\varphi_{\alpha}$ - they are written in the internal language of toposes - works for the subobject fibration $\underset{\mathbb{C}}{\stackrel{\operatorname{Sub}}{\mathbb{C}}}$ of any topos $\mathbb{C}$. Therefore, the categorical definition of coinductive predicates (Definition 2.3) allows us to interpret the language $\mathrm{R} v$ in constructive underlying logics. Suitable completeness of $\mathbb{C}$ ensures that a final $\left(c^{*} \circ \varphi\right)$-coalgebra in Definition 2.3 exists.

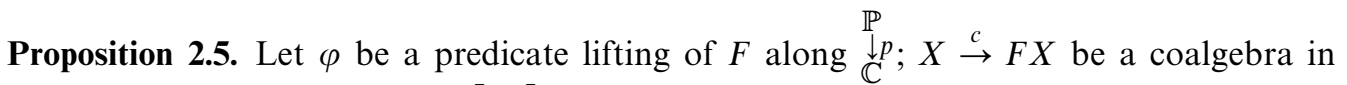
$\mathbb{C}$; and $P \in \mathbb{P}_{X}$. We have $P \leqslant \llbracket v \varphi \rrbracket_{c}$ if and only if there exists a $\varphi$-invariant $Q$ such that $P \leqslant Q$.

The proposition is trivial but potentially useful. It says that an invariant can be used as a 'witness' for a coinductive predicate. This is how bisimilarity is commonly established 
(namely by finding a bisimulation); and it can be used e.g. in Abramsky and Winschel (2015, Section 6) as an alternative to the metric coinduction principle used there. ${ }^{\dagger}$

Remark 2.6. The coalgebraic modal logic literature exploits the fact that there can be many predicate liftings (in the form of natural transformations) of the same functor $F$. Different predicate liftings correspond to different modalities (such as $\square$ vs. $\diamond$ for the same functor $\mathcal{P}$ ). This view of predicate liftings is also the current paper's concern (see Example 2.4).

In contrast, in fibrational studies like Hermida (1993), Hermida and Jacobs (1998), Fumex et al. (2011) and Atkey et al. (2012), use of predicate liftings has focussed on the validity of the (co)induction proof principle. For such purposes, it is necessary to choose a predicate lifting $\varphi$ that is 'comprehensive enough,' covering all the possible $F$-behaviours. In fact, it is common in these studies that 'the' predicate lifting, denoted by $\operatorname{Pred}(F)$, is assigned to a functor $F$. An exception is Jacobs (2010).

\section{Final sequences in a fibration}

Here, we present our main technical result (Theorem 3.9). It generalizes known behavioural $\omega$-bounds (like Hennessy and Milner (1985, Theorem 2.1); see Section 1.1); and claims that the chain (2) for a coinductive predicate stabilizes after $\omega$ steps, assuming that the behaviour type functor $F$ and the underlying logic $\underset{\mathbb{C}}{\mathbb{P}}$ are 'finitary' in a suitable sense (but no size restriction on $\varphi$ ).

\subsection{Size restrictions on a fibration}

We axiomatize finitariness conditions in the language of locally presentable categories (see Appendix B for a minimal introduction). Singling out these conditions lies at the heart of our technical contribution.

Definition 3.1 (LFP category). A category $\mathbb{C}$ is $L F P$ if it is cocomplete and it has a (small) set $\mathbb{F}$ of finitely presentable (FP) objects such that every object is a filtered colimit of objects in $\mathbb{F}$.

Definition 3.2 (Finitely determined fibration). A (poset) fibration $\underset{\mathbb{C}}{\mathbb{P}}$ is finitely determined if it satisfies the following:

1. $\mathbb{C}$ is LFP, with a set $\mathbb{F}$ of FP objects (as in Definition 3.1). $\mathbb{P}$

2. $\underset{\mathbb{C}}{\downarrow}$ has fibrewise limits and colimits (as in Definition C.9).

3. For arbitrary $X \in \mathbb{C}$, let $\left(X_{I}\right)_{I \in \mathbb{I}}$ be the canonical diagram for $X$ with respect to $\mathbb{F}$ (i.e. $\mathbb{I}=\mathbb{F} / X$, see Lemma B.4), with a colimiting cocone $\left(X_{I} \stackrel{\kappa_{I}}{\rightarrow} X\right)_{I \in \mathbb{I}}$. Then for any

$\dagger$ To be precise, only if we take PE in Abramsky and Winschel (2015) - that is in fact, a least fixed-point specification - as an atomic proposition (and that is essentially what is done in the proofs in Abramsky and Winschel (2015, Section 6)). Our future work on nested $\mu$ 's and $v$ 's will more adequately address the situation. 
$P, Q \in \mathbb{P}_{X}$

$$
P \leqslant Q \quad \Longleftrightarrow \quad \kappa_{I}^{*} P \leqslant \kappa_{I}^{*} Q \text { in } \mathbb{P}_{X_{I}} \text { for each } I \in \mathbb{I} \text {. }
$$

The intuition behind Cond. 3 is that a predicate $P \in \mathbb{P}_{X}$ (over arbitrary $X \in \mathbb{C}$ ) is determined by its restrictions $\left(\kappa_{I}^{*} P\right)_{I \in \mathbb{I}}$ to $\mathrm{FP}$ objects $X_{I}$. One convenient sufficient condition for Cond. 3 is that the total category $\mathbb{P}$ is itself LFP, with its FP objects residing above the FP objects in $\mathbb{C}$ (Corollary 6.2). We note that Cond. 1 guarantees, since LFP implies completeness, that an $\left(\omega^{\mathrm{op}}\right.$-)limit $F^{\omega} 1$ of the final $F$-sequence (4) exists. However, this does not mean (nor do we need) that $F^{\omega} 1$ carries a final $F$-coalgebra; it fails for $F=\mathcal{P}_{\omega}$, see Worrell (2005).

Definition 3.3 (Well-founded fibration). A well-founded fibration is a finitely determined fibration that further satisfies the following:

4. If $X \in \mathbb{F}$ (hence FP), the fibre $\mathbb{P}_{X}$ is such that: the category $\mathbb{P}_{X}^{\text {op }}$ consists solely of FP objects.

Since $\mathbb{P}_{X}$ is complete, this is equivalent to: there is no $\left(\omega^{\text {op }}\right.$ - $)$ chain $P_{0}>P_{1}>\cdots$ in $\mathbb{P}_{X}$ that is strictly descending.

We note that the following stronger variant of the condition 3.3 rarely holds:

$3.3^{\prime}:$ For any $X \in \mathbb{C}$, there is no strictly descending $\omega^{\text {op }}$-chain in $\mathbb{P}_{X}$

(it fails in $\begin{gathered}\text { Pred } \\ \text { Sets }\end{gathered}$ ). The original Cond. 3.3 holds in many examples (as we will see later in Section 7), thanks to the restriction that $X$ is FP.

Remark 3.4. Conditions $3-3.3$ mention a fixed set $F$ of FP objects. It is not hard to see that this is not necessary, and we can take as $\mathbb{F}$ the set of all FP objects without loss of generality. (Stating the conditions in terms of $\mathbb{F}$ is an advantage when it comes to checking them, though.)

Let us first note that, by Adámek and Rosický (1994, Remark 1.9), any FP object $Y \in \mathbb{C}$ is a split quotient of some $X \in \mathbb{F}$, i.e. there exists $q: X \rightarrow Y$ and $i: Y \mapsto X$ with $q \circ i=\mathrm{id}_{Y}$.

Then we indeed have the following. On Cond. 3, for an FP object $Y$ and $\kappa^{\prime}: Y \rightarrow X$, take $X^{\prime} \in \mathbb{F}$ with a splitting $X^{\prime} \stackrel{q}{\rightarrow} Y \stackrel{i}{\mapsto} X^{\prime}$. Then we can take $I$ such that $X_{I}=X^{\prime}$ and $\kappa_{I}=\kappa^{\prime} \circ q$. Hence, $\kappa_{I}^{*} P \leqslant \kappa_{I}^{*} Q$ in $\mathbb{P}_{X_{I}}$ induces $\kappa^{\prime *} P \leqslant \kappa^{\prime *} Q$ in $\mathbb{P}_{Y}$ because $\kappa^{\prime}=\kappa_{I} \circ i$. On Cond. 3.3, for an FP object $Y$, take $X \in \mathbb{F}$ with a splitting $X \stackrel{q}{\rightarrow} Y \stackrel{i}{\rightarrow} X$. Then a strictly decreasing chain $Q_{0}>Q_{1}>\cdots$ in $\mathbb{P}_{Y}$ induces a strictly decreasing chain $q^{*} Q_{0}>q^{*} Q_{1}>\cdots$ in $\mathbb{P}_{X}$. Here, the strictness of the latter is by $i^{*} q^{*} Q_{n}=Q_{n}$.

The following trivial fact is written down for the record.

Lemma 3.5. A finitely determined fibration $\underset{\mathbb{C}}{\mathbb{P}}$ is well-founded if $\mathbb{P}_{X}$ is a finite category for each $X \in \mathbb{F}$. 

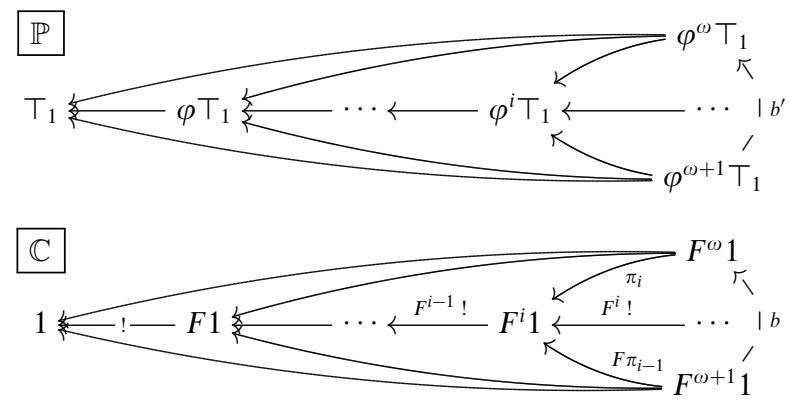

Fig. 1. Final sequences in a fibration.

\subsection{Final sequences in a fibration}

The following result from Jacobs (1999, Proposition 9.2.1) is crucial in our development.

Lemma 3.6. Let $\underset{\mathbb{C}}{\mathbb{P}}$ be a fibration, with $\mathbb{C}$ being complete. Then $p$ has fibrewise limits if and only if $\mathbb{P}$ is complete and $p: \mathbb{P} \rightarrow \mathbb{C}$ preserves limits. If this is the case, a limit of a small diagram $\left(P_{I}\right)_{I \in \mathbb{I}}$ in $\mathbb{P}$ can be given by

$$
\bigwedge_{I \in \mathbb{I}}\left(\pi_{I}^{*} P_{I}\right) \quad \text { over } \operatorname{Lim}_{I \in \mathbb{I}} X_{I}
$$

Here, $X_{I}:=p P_{I} ;\left(\operatorname{Lim}_{I \in \mathbb{I}} X_{I} \stackrel{\pi_{I}}{\rightarrow} X_{I}\right)_{I \in \mathbb{I}}$ is a limiting cone in $\mathbb{C}$; and $\bigwedge_{I \in \mathbb{I}}$ denotes the the inf in the fibre $\mathbb{P}_{\text {Lim }_{I} X_{I}}$. Moreover, $\bigwedge_{I \in \mathbb{I}}\left(\pi_{I}^{*} P_{I}\right)$ is a limit of the diagram of shape $\mathbb{I}$, namely $\pi_{I}^{*} P_{I} \leqslant \pi_{J}^{*} P_{J}$ holds for any $I \rightarrow J$ in $\mathbb{I}$.

Figure 1 presents two sequences. Here, we assume that $\underset{\mathbb{C}}{\mathbb{P}}$ is finitely determined (Definition 3.2) and that $\varphi$ is a predicate lifting of $F$. In the bottom diagram (in $\mathbb{C}$ ), the object $1 \in \mathbb{C}$ is a final one (it exists since LFP implies completeness); $F 1 \stackrel{!}{\rightarrow} 1$ is the unique map; $F^{\omega+1} 1:=F\left(F^{\omega} 1\right)$; and $b$ is a unique mediating arrow to the limit $F^{\omega} 1$. In the top diagram (in $\mathbb{P}$ ), the object $T_{1}$ is the final object in the fibre $\mathbb{P}_{1}$; by Lemma 3.6, this is precisely a final object in the total category $\mathbb{P}$. Hence, this diagram is nothing but a final sequence for the functor $\varphi$ in $\mathbb{P}$. A limit $\varphi^{\omega} \top_{1}$ of this final sequence exists, again by Lemma 3.6; and moreover, it can be chosen above $F^{\omega} 1$. We define $\varphi^{\omega+1} \top_{1}:=\varphi\left(\varphi^{\omega} \top_{1}\right)$.

Lemma 3.7 (Key lemma). Let $\underset{\mathbb{C}}{\mathbb{P}}$ be a well-founded fibration; $F: \mathbb{C} \rightarrow \mathbb{C}$ be finitary; and $\varphi$ be a predicate lifting of $F$. Then the final $\varphi$-sequence 'stabilizes' after $\omega$ steps (modulo reindexing via $b$ ). Precisely, in Figure 1, we have $\varphi^{\omega+1} \top_{1}=b^{*}\left(\varphi^{\omega} \top_{1}\right)$.

Proof. We proceed by steps.

Step a. We observe that, in Figure 1, the top diagram is carried to the one below by the functor $p: \mathbb{P} \rightarrow \mathbb{C}$. This is straightforward: The arrow $\varphi \top_{1} \rightarrow \top_{1}$ must be carried to the unique arrow $!: F 1 \rightarrow 1$; on the mediating arrow $b^{\prime}$ in $\mathbb{P}$, since $p b^{\prime}$ is again a mediating arrow in $\mathbb{C}$, it must coincide with $b$. 
Step b. Before moving on, we observe that Cond. 3 in Definition 3.2 yields a seemingly stronger statement (Cond. 3' below).

Sublemma 3.8. For a finitely determined fibration $\underset{\mathbb{C}}{\mathbb{P}}$ the following holds:

$3^{\prime}$ Let $X \in \mathbb{C} ; P, Q \in \mathbb{P}_{X}$; and $\left(Y_{J}\right)_{J \in \mathbb{J}}$ be an arbitrary filtered diagram in $\mathbb{C}$ such that $\operatorname{Colim}_{J} Y_{J}=X$, with a colimiting cocone $\left(Y_{J} \stackrel{\gamma_{J}}{\rightarrow} X\right)_{J \in J}$. Then $P \leqslant Q$ if and only if for each $J \in \mathbb{J}, \gamma_{J}^{*} P \leqslant \gamma_{J}^{*} Q$ in $\mathbb{P}_{Y_{J}}$.

Proof. (Of Sublemma 3.8) The only non-trivial statement is the 'if' part of the direction $3 \Rightarrow 3^{\prime}$. It suffices to show that $\gamma_{J}^{*} P \leqslant \gamma_{J}^{*} Q$ (for each $J \in \mathbb{J}$ ) implies $\kappa_{I}^{*} P \leqslant \kappa_{I}^{*} Q$ (for each $I \in \mathbb{I}$ ), where $\kappa_{I}$ and $\mathbb{I}$ are as in Cond. 3 .

Let $I \in \mathbb{I}$. Since $X_{I}$ is FP, an arrow $\kappa_{I}: X_{I} \rightarrow X$ to a filtered colimit $X=\operatorname{Colim}_{J} Y_{J}$ factors through some $Y_{J_{I}} \stackrel{\gamma_{J_{I}}}{\rightarrow} X$, as in the diagram below.

$$
X_{I} \underset{h_{I}}{\rightleftharpoons}{ }_{Y_{J_{I}}} \underset{\gamma_{J_{I}}}{\longrightarrow} X=\operatorname{Colim}_{J} Y_{J}
$$

Now, we have $\kappa_{I}^{*} P=h_{I}^{*} \gamma_{J_{I}}^{*} P \leqslant h_{I}^{*} \gamma_{J_{I}}^{*} Q=\kappa_{I}^{*} Q$, where the inequality is by the assumption that $\gamma_{J}^{*} P \leqslant \gamma_{J}^{*} Q$ for each $J \in \mathbb{J}$. This proves Sublemma 3.8.

Step c. By Step a, we see that $\varphi^{\omega+1} T_{1} \leqslant b^{*}\left(\varphi^{\omega} T_{1}\right)$ by the universality of a Cartesian arrow. In what follows we shall prove its converse:

$$
b^{*}\left(\varphi^{\omega} \top_{1}\right) \leqslant \varphi^{\omega+1} \top_{1} \quad \text { in } \mathbb{P}_{F^{\omega+1} 1} .
$$

Let us take a filtered diagram $\left(X_{I}\right)_{I \in \mathbb{I}}$ in $\mathbb{C}$ such that $X_{I} \in \mathbb{F}$ (for each $I \in \mathbb{I}$ ) and $F^{\omega} 1=\operatorname{Colim}_{I \in \mathbb{I}} X_{I}$, with $\left(X_{I} \stackrel{\kappa_{I}}{\rightarrow} F^{\omega} 1\right)_{I \in \mathbb{I}}$ being the colimiting cocone. Then we have

$$
F^{\omega+1} 1=F\left(\underset{I \in \mathbb{I}}{\operatorname{Colim}} X_{I}\right)=\underset{I \in \mathbb{I}}{\operatorname{Colim}} F X_{I},
$$

by the assumption that $F$ is finitary; moreover, $\left(F X_{I} \stackrel{F \kappa_{I}}{\rightarrow} F^{\omega+1} 1\right)_{I \in \mathbb{I}}$ is a colimiting cocone. The diagram $\left(X_{I}\right)_{I \in \mathbb{I}}$ is filtered, and so is the latter diagram $\left(F X_{I}\right)_{I \in \mathbb{I}}$. Thus, by Cond. $3^{\prime}$ in Sublemma 3.8, showing the following proves Equation (8):

$$
\left(F \kappa_{I}\right)^{*}\left(b^{*}\left(\varphi^{\omega} \top_{1}\right)\right) \leqslant\left(F \kappa_{I}\right)^{*}\left(\varphi^{\omega+1} \top_{1}\right) \text { for each } I \in \mathbb{I} .
$$

Step d. To prove Equation (9), we first prove the following fact: For each $I \in \mathbb{I}$, there exists $i_{I} \in \omega$ such that

$$
\kappa_{I}^{*}\left(\varphi^{\omega} \top_{1}\right)=\kappa_{I}^{*}\left(\pi_{i_{I}}^{*}\left(\varphi^{i_{I}} \top_{1}\right)\right) \quad \text { in } \mathbb{P}_{X_{I}} .
$$

That is, the final sequence in $\mathbb{P}$ (Figure 1), when restricted to $X_{I}$ (that is FP), stabilizes within finitely many steps. Indeed, by Lemma 3.6, the $\omega^{\text {op }}$-limit $\varphi^{\omega} \top_{1}$ is described as an

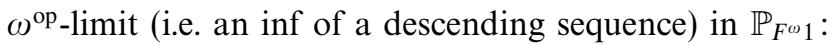

$$
\varphi^{\omega} \top_{1}=\bigwedge_{i \in \omega} \pi_{i}^{*}\left(\varphi^{i} \top_{1}\right)
$$

Therefore, we have $\kappa_{I}^{*}\left(\varphi^{\omega} \top_{1}\right)=\bigwedge_{i \in \omega} \kappa_{I}^{*} \pi_{i}^{*}\left(\varphi^{i} \top_{1}\right)$ since reindexing $\kappa_{I}^{*}$ preserves fibrewise limits $\bigwedge$. Here, the sequence $\left(\kappa_{I}^{*} \pi_{i}^{*}\left(\varphi^{i} \top_{1}\right)\right)_{i \in \omega}$ in $\mathbb{P}_{X_{I}}$ is also descending. Therefore, by $p$ 
being a well-founded fibration (Definition 3.3) and $X_{I}$ being FP, there exists $i_{I} \in \omega$ at which the descending sequence $\left(\kappa_{I}^{*} \pi_{i}^{*}\left(\varphi^{i} \top_{1}\right)\right)_{i \in \omega}$ in $\mathbb{P}_{X_{I}}$ stabilizes, that is,

$$
\kappa_{I}^{*}\left(\bigwedge_{i \in \omega} \pi_{i}^{*}\left(\varphi^{i} \top_{1}\right)\right)=\bigwedge_{i \in \omega} \kappa_{I}^{*} \pi_{i}^{*}\left(\varphi^{i} \top_{1}\right)=\kappa_{I}^{*}\left(\pi_{i_{I}}^{*}\left(\varphi^{i_{I}} \top_{1}\right)\right) \quad \text { in } \mathbb{P}_{X_{I}}
$$

Combined with Equation (11), this proves Equation (10).

Step e. Finally, let us prove Equation (9). For each $I \in \mathbb{I}$,

$$
\begin{aligned}
\left(F \kappa_{I}\right)^{*}\left(b^{*}\left(\varphi^{\omega} \top_{1}\right)\right)= & \left(F \kappa_{I}\right)^{*}\left(b^{*}\left(\bigwedge_{i \in \omega} \pi_{i}^{*}\left(\varphi^{i} \top_{1}\right)\right)\right) \quad \text { by Equation (11) } \\
= & \bigwedge_{i \in \omega}\left(F \kappa_{I}\right)^{*}\left(b^{*}\left(\pi_{i}^{*}\left(\varphi^{i} \top_{1}\right)\right)\right) \quad \text { reindexing preserves } \bigwedge \\
\leqslant & \bigwedge_{j \in \omega}\left(F \kappa_{I}\right)^{*}\left(b^{*}\left(\pi_{j+1}^{*}\left(\varphi^{j+1} \top_{1}\right)\right)\right) \quad \text { letting } i=j+1 \text { for } i \geqslant 1 \\
= & \left.\bigwedge_{j \in \omega}\left(F \kappa_{I}\right)^{*}\left(\left(F \pi_{j}\right)^{*}\left(\varphi^{j+1} \top_{1}\right)\right) \quad \text { by } \pi_{j+1} \circ b=F \pi_{j} \text { (see Figure } 1\right) \\
= & \bigwedge_{j \in \omega} \varphi\left(\kappa_{I}^{*} \pi_{j}^{*}\left(\varphi^{j} \top_{1}\right)\right) \quad \text { by Definition } 2.2 \\
\leqslant & \varphi\left(\kappa_{I}^{*} \pi_{i_{I}}^{*}\left(\varphi^{i_{I}} \top_{1}\right)\right) \quad \text { letting } j=i_{I} \text { on the LHS } \\
= & \varphi\left(\kappa_{I}^{*}\left(\varphi^{\omega} \top_{1}\right)\right) \quad \text { by Equation }(10) \\
= & \left(F \kappa_{I}\right)^{*}\left(\varphi^{\omega+1} \top_{1}\right) \quad \text { by Definition } 2.2 \text { and } \varphi^{\omega+1} \top_{1}=\varphi\left(\varphi^{\omega} \top_{1}\right) .
\end{aligned}
$$

This proves Equation (9) and concludes the proof of Lemma 3.7.

The object $\varphi^{\omega} \top_{1}$ is a 'prototype' of $\varphi$-coinductive predicates in various coalgebras. This is part of the main theorem below.

It is standard that a coalgebra $X \stackrel{c}{\rightarrow} F X$ in $\mathbb{C}$ induces a cone over the final $F$-sequence, and hence a mediating arrow $X \rightarrow F^{\omega} 1$ (see below). Concretely, $c_{i}: X \rightarrow F^{i} 1$ is defined inductively by $X \stackrel{c_{0}}{\rightarrow} 1$ is !; and $c_{i+1}$ is the composite $X \stackrel{c}{\rightarrow} F X \stackrel{F c_{i}}{\rightarrow} F^{i+1} 1$. The induced arrow to the limit $F^{\omega} 1$ is denoted by $c_{\omega}$.

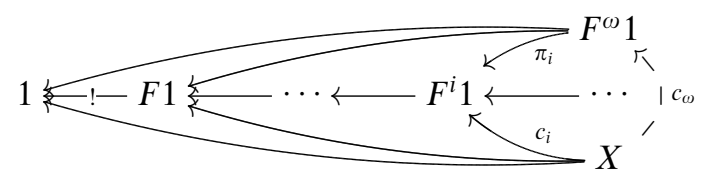

Note that $F^{\omega} 1$ does not necessarily carry a final $F$-coalgebra (see Remark 3.12 ).

Theorem 3.9 (Main result). Let $\underset{\mathbb{C}}{\mathbb{P}}$ be a well-founded fibration; $F: \mathbb{C} \rightarrow \mathbb{C}$ be a finitary functor; $\varphi$ be a predicate lifting of $F$ along $p$; and $X \stackrel{c}{\rightarrow} F X$ be a coalgebra in $\mathbb{C}$.

1. The $\varphi$-coinductive predicate $\llbracket v \varphi \rrbracket_{c}$ in $c$ (Definition 2.3 ) exists. It is obtained by the following reindexing of $\varphi^{\omega} \top_{1}$, where $c_{\omega}$ is the mediating map in Equation (12).

$$
\llbracket v \varphi \rrbracket_{c}=c_{\omega}^{*}\left(\varphi^{\omega} \top_{1}\right)
$$


2. Moreover, the predicate $\llbracket v \varphi \rrbracket_{c}$ is the limit of the following $\omega^{\mathrm{op}}$-chain in the fibre $\mathbb{P}_{X}$

$$
\top_{X} \geqslant\left(c^{*} \circ \varphi\right)\left(\top_{X}\right) \geqslant\left(c^{*} \circ \varphi\right)^{2}\left(\top_{X}\right) \geqslant \cdots,
$$

that stabilizes after $\omega$ steps. That is, $\llbracket v \varphi \rrbracket_{c}=\bigwedge_{i \in \omega}\left(c^{*} \circ \varphi\right)^{i}\left(\top_{X}\right)$.

Proof. We proceed by steps.

Step a. We first show that the descriptions of $\llbracket v \varphi \rrbracket_{c}$ in the items 1-2 are the same:

$$
c_{\omega}^{*}\left(\varphi^{\omega} \top_{1}\right)=\bigwedge_{i \in \omega}\left(c^{*} \circ \varphi\right)^{i}\left(\top_{X}\right)
$$

We have

$$
\begin{aligned}
c_{\omega}^{*}\left(\varphi^{\omega} \top_{1}\right) & =c_{\omega}^{*}\left(\bigwedge_{i \in \omega} \pi_{i}^{*}\left(\varphi^{i} \top_{1}\right)\right) \quad \text { by Lemma } 3.6 \\
& =\bigwedge_{i \in \omega} c_{\omega}^{*}\left(\pi_{i}^{*}\left(\varphi^{i} \top_{1}\right)\right) \quad \text { since reindexing preserves } \bigwedge \\
& =\bigwedge_{i \in \omega} c_{i}^{*}\left(\varphi^{i} \top_{1}\right) \quad \text { by the definition of } c_{\omega} .
\end{aligned}
$$

Furthermore, $c_{i}^{*}\left(\varphi^{i} \top_{1}\right)$ in the above is seen to be equal to $\left(c^{*} \circ \varphi\right)^{i}\left(\top_{X}\right)$. This is shown by induction on $i \in \omega$. For $i=0$, the claim amounts to $!^{*}\left(\top_{1}\right)=\top_{X}$, which holds since reindexing preserves $T$. For the step case,

$$
\begin{aligned}
c_{i+1}^{*}\left(\varphi^{i+1} \top_{1}\right) & =c^{*}\left(F c_{i}\right)^{*}\left(\varphi^{i+1} \top_{1}\right) \quad \text { by } c_{i+1}=F c_{i} \circ c \\
& =c^{*}\left(\varphi\left(c_{i}^{*}\left(\varphi^{i} \top_{1}\right)\right)\right) \quad \text { by Definition } 2.2 \\
& =\left(c^{*} \circ \varphi\right)\left(\left(c^{*} \circ \varphi\right)^{i}\left(\top_{X}\right)\right) \quad \text { by induction hypothesis. }
\end{aligned}
$$

Therefore the Equation (15) holds.

Step b. In order to show that $\bigwedge_{i \in \omega}\left(c^{*} \circ \varphi\right)^{i}\left(\top_{X}\right)$ is the $\varphi$-coinductive predicate in $c$, we shall exhibit that the chain (14) - the final $\left(c^{*} \circ \varphi\right)$-sequence in $\mathbb{P}_{X}$ - stabilizes after $\omega$ steps. By Equation (15), the claim $\left(c^{*} \circ \varphi\right)\left(\bigwedge_{i \in \omega}\left(c^{*} \circ \varphi\right)^{i}\left(\top_{X}\right)\right)=\bigwedge_{i \in \omega}\left(c^{*} \circ \varphi\right)^{i}\left(\top_{X}\right)$ reduces to

$$
\left(c^{*} \circ \varphi\right)\left(c_{\omega}^{*}\left(\varphi^{\omega} \top_{1}\right)\right)=c_{\omega}^{*}\left(\varphi^{\omega} \top_{1}\right) .
$$

Step c. Finally, we shall prove Equation (17):

$$
\begin{aligned}
c^{*}\left(\varphi\left(c_{\omega}^{*}\left(\varphi^{\omega} \top_{1}\right)\right)\right) & =c^{*}\left(\left(F c_{\omega}\right)^{*}\left(\varphi\left(\varphi^{\omega} \top_{1}\right)\right)\right) \quad \text { by Definition } 2.2 \\
& =c^{*}\left(\left(F c_{\omega}\right)^{*}\left(b^{*}\left(\varphi^{\omega} \top_{1}\right)\right)\right) \quad \text { by Lemma } 3.7 \\
& =\left(b \circ F c_{\omega} \circ c\right)^{*}\left(\varphi^{\omega} \top_{1}\right) \\
& =c_{\omega}^{*}\left(\varphi^{\omega} \top_{1}\right) .
\end{aligned}
$$

For the last equality, we used $b \circ F c_{\omega} \circ c=c_{\omega}$, which is proved by showing that $b \circ F c_{\omega} \circ c$ is also a mediating map in Equation (12). Indeed, for each $i \geqslant 1$,

$$
\begin{aligned}
\pi_{i} \circ b \circ F c_{\omega} \circ c & =F \pi_{i-1} \circ F c_{\omega} \circ c \text { see Figure } 1 \\
& =F c_{i-1} \circ c \text { by Equation }(12) \\
& =c_{i} \text { by the definition of } c_{i} .
\end{aligned}
$$

This concludes the proof. 
Example 3.10 $(\mathrm{R} v)$. We shall continue Example 2.4 and derive from Theorem 3.9 the behavioural bound result described in Section 1.1: the chain (2) stabilizes after $\omega$ steps, for each $\alpha \in \mathrm{R} v_{u}$ and each finitely branching Kripke model $c$.

Indeed, the latter is the same thing as a coalgebra $X \stackrel{c}{\rightarrow} F_{\mathrm{fbK}} X$, where $F_{\mathrm{fbK}}=\mathcal{P}(\mathrm{AP}) \times$ $\mathcal{P}_{\omega}\left({ }_{-}\right)$. Compared to $F_{\mathrm{K}}$ in Example 2.4, the powerset functor is restricted from $\mathcal{P}$ to $\mathcal{P}_{\omega}$; this makes $F_{\mathrm{fbK}}$ a finitary functor. Still the same definition of $\varphi_{\alpha}$ defines a predicate

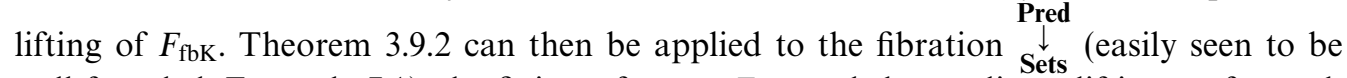
well-founded, Example 7.1), the finitary functor $F_{\mathrm{fbK}}$ and the predicate lifting $\varphi_{\alpha}$ for each $\alpha$. It is not hard to see that the function $[\alpha]_{c}: \mathcal{P} X \rightarrow \mathcal{P} X$ in Section 1.1 coincides with

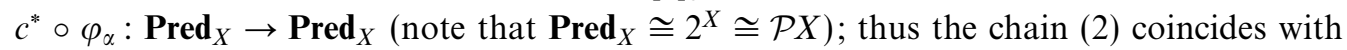
Equation (14) that stabilizes after $\omega$ steps by Theorem 3.9.

Remark 3.11. The $\omega$-bound of the length of the chain (14) is sharp.

A (counter)example is given in the setting of Example 3.10, by the predicate lifting $\varphi_{\diamond u}$ and the coalgebra (i.e. Kripke structure) $c_{2}$ below. There $b_{i, i}$ has no successors. Indeed, while $\llbracket v \varphi_{\diamond u} \rrbracket_{c_{2}}$ is $\left\{a_{i} \mid i \in \omega\right\}$, its $i$ th approximant $\left(\left(c_{2}\right)_{i}^{*} \circ \varphi_{\diamond u}^{i}\right)\left(\top_{X}\right)$ in Equation (14) contains $b_{i, 0}$ too.

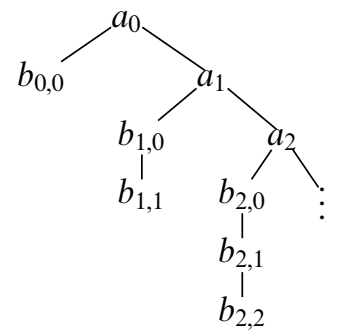

Remark 3.12. It is notable that Theorem 3.9 imposes no size restrictions on $\varphi: \mathbb{P} \rightarrow \mathbb{P}$. Being a predicate lifting is enough. To find an example such that $\varphi$ is not finitary is future work. Our main theorem would not become trivial even if it turns out that $\varphi$ is always finitary.

Final $F$-sequences are commonly used for the construction of a final $F$-coalgebra. It is not always the case, however, that the limit $F^{\omega} 1$ is itself the carrier of a final coalgebra (even for finitary $F$; see Worrell (2005, Section 5)). One obtains a final coalgebra either by (1) quotienting $F^{\omega} 1$ by the behavioural equivalence (see e.g. Pattinson (2003)); or (2) continuing the final sequence till $\omega+\omega$ steps. The latter construction is worked out 
in Worrell (2005) (in Sets) and in Adámek (2003) in LFP $\mathbb{C}$ with additional assumptions. Its relevance to the current work is yet to be investigated.

We emphasize that a final $\varphi$-sequence 'stabilizes' in $\omega$ steps relatively to the underlying final $F$-sequence. In fact, we can also show that the final $\varphi$-sequence absolutely stabilizes in $\omega+\omega$ steps for some LFP $\mathbb{C}$ including Sets; a proof can be done by observing that the final $\varphi$-sequence stabilizes as soon as the final $F$-sequence stabilizes, once we are beyond $\omega$ steps.

To show directly the stabilization of the final $\varphi$-sequence in $\omega+\omega$ steps, one may want to prove that $\mathbb{P}$ is strongly LFP as in Adámek (2003) and that $\varphi$ is finitary. Neither of these seems easy.

Coalgebra morphisms are compatible with coinductive predicates. This fact, like Proposition 2.5, is potentially useful in establishing coinductive predicates.

Proposition 3.13. Let $f: X \rightarrow Y$ be a coalgebra morphism from $X \stackrel{c}{\rightarrow} F X$ to $Y \stackrel{d}{\rightarrow} F Y$. In the setting of Lemma 3.7 and Theorem 3.9:

1. If $Q \in \mathbb{P}_{Y}$ is a $\varphi$-invariant in $d$, so is $f^{*} Q \in \mathbb{P}_{X}$ in $c$.

2. We have $\llbracket v \varphi \rrbracket_{c}=f^{*}\left(\llbracket v \varphi \rrbracket_{d}\right)$.

Proof. For the item 1,

$$
\begin{aligned}
f^{*} Q & \leqslant f^{*} d^{*}(\varphi Q) \quad Q \text { is an invariant } \\
& =c^{*}(F f)^{*}(\varphi Q) \quad f \text { is a homomorphism } \\
& =\left(c^{*} \circ \varphi\right)\left(f^{*} Q\right) \quad \text { by Definition 2.2. }
\end{aligned}
$$

For the item 2 , the coalgebras give rise to mediating arrows $X \stackrel{c_{\omega}}{\rightarrow} F^{\omega} 1$ and $Y \stackrel{d_{\omega}}{\rightarrow} F^{\omega} 1$, respectively, as in Equation (12). It is easy to see that $c_{\omega}=d_{\omega} \circ f$ (using the universality of the limit $F^{\omega} 1$ ); using Equation (13) the claim follows.

Remark 3.14. The current paper focusses on FP objects, finitary functors, etc. - i.e. the $\omega$-presentable setting (see Adámek and Rosický (1994, Section 1.B)). This is for the simplicity of presentation: the results, as usual (as e.g. in Klin (2007)), can be easily generalized to the $\lambda$-presentable setting for an arbitrary regular cardinal $\lambda$. In such an extended setting, we obtain a behavioural $\lambda$-bound.

\section{A fibration of invariants}

We organize the above observations in a more abstract fibred setting. The technical results are mostly standard; see e.g. Hermida (1993), Hermida and Jacobs (1998) and Jacobs (2012, Chap.6).

We write $\operatorname{Coalg}(F)$ for the category of $F$-coalgebras.

Proposition 4.1. Let $\varphi$ be a predicate lifting of $F$ along $\underset{\mathbb{C}}{\mathbb{P}}$. Then the fibration $\underset{\mathbb{C}}{\mathbb{P}}$ is lifted $\operatorname{Coalg}(\varphi)$

to a fibration $\begin{gathered}\downarrow \bar{p} \\ \operatorname{Coalg}(F)\end{gathered}$, with two forgetful functors forming a map of fibrations from the latter to the former. 
Proof. It is easy to check each fibre $\operatorname{Coalg}(\varphi)_{X \stackrel{c}{\rightarrow} F X}$ is a poset. Let $(X \stackrel{c}{\rightarrow} F X) \stackrel{f}{\rightarrow}(Y \stackrel{d}{\rightarrow}$ $F Y$ ) be an arrow in Coalg $(F)$, and $P \stackrel{s}{\rightarrow} \varphi P$ be above $Y \stackrel{d}{\rightarrow} F Y$. A Cartesian lifting of $f$ is obtained as in the following diagram:

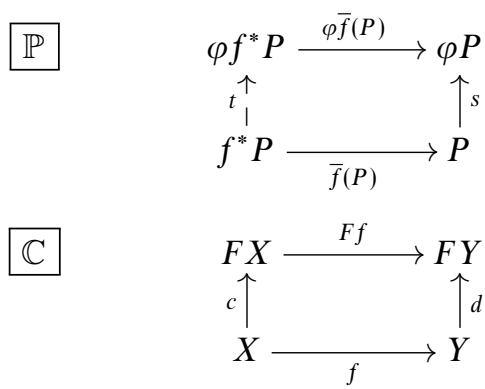

Here, we used the universality of the Cartesian lifting $\varphi \bar{f}(P)$ (see Definition 2.2).

The two forgetful functors constitute a map of fibrations: The commutativity (5) is $\operatorname{Coalg}(\varphi)$

obvious, and Cartesian liftings in $\underset{\mathbf{C o a l g}(F)}{\downarrow \vec{p}}$ (which we constructed above) are based on the Cartesian liftings in $\underset{\mathbb{C}}{\stackrel{\mathbb{P}}{\downarrow}}$.

The next observation explains the current section's title.

Proposition 4.2. Let $\begin{aligned} & \operatorname{Coalg}(\varphi) \\ & \operatorname{Coalg}(F)\end{aligned}$ be the lifted fibration in Proposition 4.1. For each coalgebra $X \stackrel{c}{\rightarrow} F X$, the fibre over $c$ coincides with the poset of $\varphi$-invariants in $c$. That is,

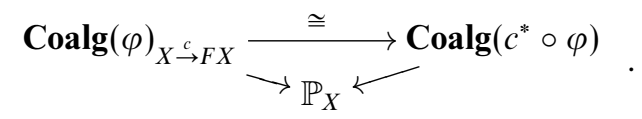

Proof. Given a $\varphi$-coalgebra $P \stackrel{s}{\rightarrow} \varphi P$ above $X \stackrel{c}{\rightarrow} F X$, we use the universality of the Cartesian lifting of $c$ to obtain a $\left(c^{*} \circ \varphi\right)$-coalgebra as in the following diagram:

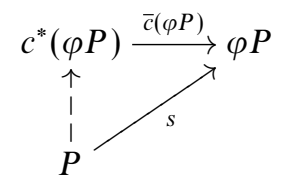

Conversely, given a $\left(c^{*} \circ \varphi\right)$-coalgebra $Q \stackrel{t}{\rightarrow} c^{*}(\varphi Q)$, we obtain a $\varphi$-coalgebra above $X \stackrel{c}{\rightarrow} F X$ as the following composite:

$$
\begin{aligned}
& c^{*}(\varphi Q) \stackrel{\bar{c}(\varphi Q)}{\longrightarrow} \varphi Q \\
& t \uparrow \\
& Q
\end{aligned}
$$

Then it is straightforward to see that the mappings are monotone and inverse to each other. The mappings commute with the forgetful functors since they do not change the carriers. 
Therefore, Theorem 3.9.1 and Proposition 3.13.2 state the fibration $\begin{gathered}\operatorname{Coalg}(\varphi) \\ \operatorname{Coalg}(F)\end{gathered}$ has fibrewise final objects. (At least part of) this statement itself is shown quite easily using the Knaster-Tarski theorem (each fibre is a complete lattice). Our contribution is their concrete construction as $\omega^{\mathrm{op}}$-limits (Theorem 3.9.2).

The following lemma is essentially a special case of Lemma 3.6, but see also Jacobs (1999, Proposition 9.2.1 and Exercise 9.2.4).

Lemma 4.3. Let $\underset{\mathbb{C}}{\mathbb{P}}$ be a fibration; and assume that $\mathbb{C}$ has a final object. Then $\underset{\mathbb{C}}{\mathbb{P}}$ has a fibrewise final object if and only if $\mathbb{P}$ has a final object that is above the final object of $\mathbb{C}$.

By applying the lemma to $\begin{gathered}\operatorname{Coalg}(\varphi) \\ \downarrow \\ \operatorname{Coalg}(F)\end{gathered}$, we obtain a basic relationship between coinductive predicates and final coalgebras.

Corollary 4.4. Let $\varphi$ be a predicate lifting of $F$ along $\underset{\mathbb{C}}{\mathbb{P}}$; and assume that a final $F$-coalgebra exists. The following are equivalent:

1. The coinductive predicate $\llbracket v \varphi \rrbracket_{c}$ exists for each coalgebra $c: X \rightarrow F X$. Moreover, they are preserved by reindexing (along coalgebra morphisms).

2. There exists a final $\varphi$-coalgebra that is above the final $F$-coalgebra.

As noted in Remark 3.12, however, our concrete construction of coinductive predicates does not rely on a final $F$-coalgebra.

\section{Inductive predicates over coinductive datatypes}

The central topic of the current paper is coinductive predicates over coinductive datatypes, the latter identified as coalgebras in the base category $\mathbb{C}$ of a fibration $\underset{\mathbb{C}}{\mathbb{P}}$. Some variations are possible, namely: inductive/coinductive predicates over inductive/coinductive datatypes. For example, Hermida and Jacobs (1998) focus on: inductive predicates over inductive datatypes (the latter identified as algebras); and coinductive predicates over coinductive datatypes (as we have done in the previous sections).

It turns out that, among these four variations, inductive predicates over coinductive datatypes allow a straightforward adaptation of our current categorical framework by taking the fibrewise opposite $\underset{\mathbb{C}}{\underset{\mathbb{P}}{\downarrow} p^{(\mathrm{op})}}$ of the fibration $\underset{\mathbb{C}}{\stackrel{\mathbb{P}}{\downarrow}}$ we are interested in. We present these results in the current section. The study of the other two variations - inductive predicates over inductive datatypes, and coinductive predicates over inductive datatypes - is left as future work. In fact, we have preliminary observations that under certain assumptions these two variations coincide. Their details will be presented in another venue.

The following is the definition of an inductive predicate (on a coinductive datatype). It is not hard to see that the definition generalizes e.g. the semantics of the $\mu$ operator of the modal $\mu$-calculus in a Kripke model. Later in Lemma 5.4, we will identify it as a coinductive predicate in the fibrewise opposite. 
Definition 5.1 (Inductive predicate). Let $\varphi$ be a predicate lifting along a fibration $\underset{\mathbb{C}}{\stackrel{\mathbb{P}}{\downarrow} \text {; and }}$ $X \stackrel{c}{\rightarrow} F X$ be a coalgebra in $\mathbb{C}$. The $\varphi$-inductive predicate in $c$ is the initial $\left(c^{*} \circ \varphi\right)$-algebra (if it exists). We denote its carrier by $\llbracket \mu \varphi \rrbracket_{c}$. Hence, it is the smallest predicate $P \in \mathbb{P}_{X}$ such that $P \geqslant c^{*}(\varphi P)$ in $\mathbb{P}_{X}$.

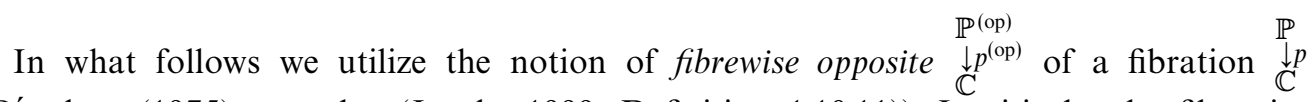
(Bénabou (1975); see also (Jacobs 1999, Definition 1.10.11)). Intuitively, the fibrewise opposite $p^{(\mathrm{op})}$ is obtained by opposing the order in each fibre $\mathbb{P}_{X}$ but leaving the base category $\mathbb{C}$, as well as the reindexing structure, as in the original fibration $p$. The precise definition is best stated via indexed categories and the Grothendieck construction. It is left to the appendix (Lemma C.13).

Some remarks are in order. First, the total category $\mathbb{P}^{(\mathrm{op})}$ of the fibrewise opposite $\underset{\mathbb{C}}{\mathbb{P}^{(\mathrm{op})}}$ is in general different from the opposite category $\mathbb{P}^{\text {op }}$ (in the usual sense) of $\mathbb{P}$. The same applies to the functor $p^{(\mathrm{op})}$, that is different from the opposite functor $p^{\mathrm{op}}$. We emphasize that in the fibrewise opposite $\underset{\mathbb{C}}{\stackrel{\mathbb{C}}{p^{(\mathrm{op})}}}$, the base category $\mathbb{C}$ stays the same. We also note that

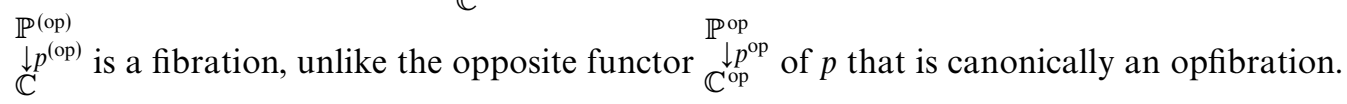

Notation 5.2. For distinction, we denote reindexing functors in fibrations $\underset{\mathbb{C}}{\mathbb{P}}$ and $\underset{\mathbb{C}}{\downarrow} \underset{p^{(\mathrm{op})}}{\mathbb{P}}$ by $f^{*}$ and $f^{\#}$, respectively. They are in fact the same monotone functions between fibres as posets:

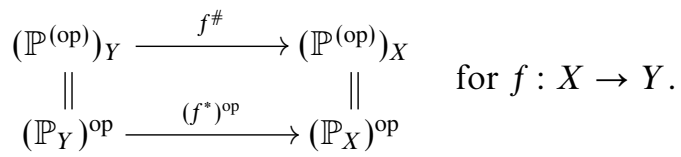

The following result, although straightforward, is essential for the subsequent technical development.

Lemma 5.3. Let $\underset{\mathbb{C}}{\stackrel{\mathbb{P}}{\downarrow}}$ be a fibration and $F$ be an endofunctor on $\mathbb{C}$. For a predicate lifting $\varphi: \mathbb{P} \rightarrow \mathbb{P}$ of $F$ along $p$, there exists a canonical predicate $\operatorname{lifting}_{\mathbb{P}(\mathrm{op})} \varphi^{(\mathrm{op})}: \mathbb{P}^{(\mathrm{op})} \rightarrow \mathbb{P}^{(\mathrm{op})}$, which we call the fibrewise opposite of $\varphi$, of $F$ along the fibration $\underset{\mathbb{C}}{\stackrel{\mathbb{P}}{\text { (op) }} p^{(\mathrm{op})}}$.

Proof. We give an explicit construction here, although the statement is almost trivial when stated in terms of indexed categories.

On objects, we define $\varphi^{(\mathrm{op})} P=\varphi P$. For the action on arrows, we first note that an arrow $P \rightarrow Q$ in $\mathbb{P}^{(\text {op })}$ above $f: X \rightarrow Y$ exists if and only if $P \leqslant f^{\#} Q$ in $\left(\mathbb{P}^{(\text {op })}\right)_{X}=\left(\mathbb{P}_{X}\right)^{\text {op }}$. Exploiting this fact, $\varphi^{(\mathrm{op})}$ 's action on the arrow $P \rightarrow Q$ is defined to be the unique arrow $\varphi^{(\mathrm{op})} P \rightarrow \varphi^{(\mathrm{op})} Q$ above $F f: F X \rightarrow F Y$. The last (unique) arrow exists, indeed: We have $\varphi^{(\mathrm{op})} P \leqslant(F f)^{\#} \varphi^{(\mathrm{op})} Q$ in $\left(\mathbb{P}^{(\mathrm{op})}\right)_{F X}$ by $\varphi P \geqslant \varphi f^{*} Q=(F f)^{*} \varphi Q$ in $\mathbb{P}_{F X}$. Here, the last equality is because $\varphi$ is a predicate lifting. 
Lemma 5.4. Let $P$ be a predicate over $X \in \mathbb{C}$.

1. The object $P \in \mathbb{P}_{X}$ carries a $\left(c^{*} \circ \varphi\right)$-algebra if and only if $P \in\left(\mathbb{P}_{X}\right)^{\mathrm{op}}=\left(\mathbb{P}^{(\mathrm{op})}\right)_{X}$ is a $\varphi^{(\mathrm{op})}$-invariant in $c$.

2. The $\varphi$-inductive predicate in $c$ is the $\varphi^{(\mathrm{op})}$-coinductive predicate in $c$. That is, $\llbracket \mu \varphi \rrbracket_{c}=$ $\llbracket v\left(\varphi^{(\mathrm{op})}\right) \rrbracket_{c}$ as objects in $\mathbb{P}_{X}$.

Proof. The category of $\left(c^{*} \circ \varphi\right)$-algebras in $\mathbb{P}_{X}$ is dually equivalent to the category of $\left(c^{\#} \circ \varphi^{(\mathrm{op})}\right)$-coalgebras in $\left(\mathbb{P}^{(\mathrm{op})}\right)_{X}$, since the following diagram (in Posets) commutes.

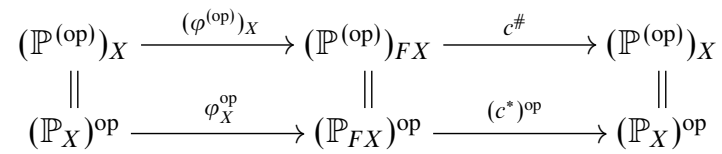

Thanks to the previous characterization - inductive predicates in $\underset{\mathbb{C}}{\mathbb{P}}$ as coinductive ones $\mathbb{P}^{(\mathrm{op})}$

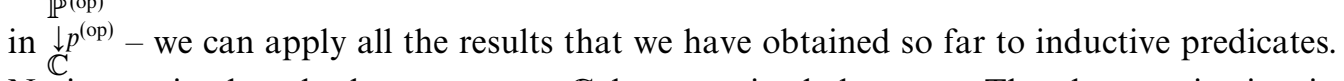
Notice again that the base category $\mathbb{C}$ has remained the same. The characterization in Lemma 5.4 can be seen as a generalization of the duality $\mu u . \varphi(u)=\neg v u . \neg \varphi(\neg u)$ between least and greatest fixed points in classical logics - the latter is a special case where fibres

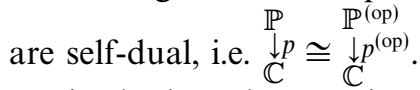

Via the last characterization, our main result (Theorem 3.9) can also be used to show the stabilization of the $\omega$-chain when calculating inductive predicates (see Corollary 5.8). The inductive predicate on $F^{\omega} 1$ is not a limit nor a colimit in $\mathbb{P}$, but it is a limit in $\mathbb{P}^{\text {(op) }}$ (see Definition 5.7).

Definition 5.5 (Co-well-founded fibration). A co-well-founded fibration is a finitely determined fibration that further satisfies:

$\overline{3.3}$. If $X \in \mathbb{F}$ (hence FP), the fibre $\mathbb{P}_{X}$ is such that: the category $\mathbb{P}_{X}$ consists solely of FP objects.

Since $\mathbb{P}_{X}$ is cocomplete, this is equivalent to: there is no $\left(\omega\right.$-)chain $P_{0}<P_{1}<\cdots$ in $\mathbb{P}_{X}$ that is strictly ascending.

Lemma 5.6. For a finitely determined fibration $\underset{\mathbb{C}}{\stackrel{\mathbb{P}}{\downarrow}}$, its fibrewise opposite $\underset{\mathbb{C}}{\stackrel{\mathbb{P}}{\downarrow} p^{(\mathrm{op})}}$ is also finitely determined. Moreover, $p^{(\mathrm{op})}$ is well-founded if and only if the fibration $p$ is cowell-founded.

Proof. It is trivial that the fibration $p^{(\mathrm{op})}$ satisfies the condition 1 (of Definition 3.2) if and only if $p$ satisfies it. For the condition $2, p^{(\mathrm{op})}$ has fibrewise limits and colimits, because $p$ has fibrewise colimits and limits, respectively. The condition 3 for $p^{(\mathrm{op})}$ is obviously equivalent to the one for $p$ since reindexing functors $\kappa_{I}^{*}, \kappa_{I}^{\#}$ are the same as functions. By $\left(\mathbb{P}^{(\mathrm{op})}\right)_{X}=\left(\mathbb{P}_{X}\right)^{\mathrm{op}}, p^{(\mathrm{op})}$ satisfies the condition 3.3 if and only if $p$ satisfies $\overline{3.3}$. 
Definition 5.7. Let $\perp_{1}$ be the least element of the fibre $\mathbb{P}_{1}$ (hence the greatest in $\left.\left(\mathbb{P}^{(\mathrm{op})}\right)_{1}\right)$. We denote by $\varphi^{\omega} \perp_{1} \in \mathbb{P}_{F^{\omega} 1}$ the limit of the following diagram in $\mathbb{P}^{(\mathrm{op})}$. It is easily seen to reside above the final $F$-sequence in $\mathbb{C}$.

$$
\perp_{1} \longleftarrow \varphi \perp_{1} \longleftarrow \cdots \longleftarrow \varphi^{i} \perp_{1} \longleftarrow \cdots \quad \text { in } \mathbb{P}^{(\mathrm{op})}
$$

Note here that $\perp_{1}$ is the final object in $\mathbb{P}^{(\mathrm{op})}$, and the object $\varphi \perp_{1}$ is the functor $\varphi^{(\mathrm{op})}$ applied to $\perp_{1}$. Therefore, the above diagram is the final $\varphi^{(\mathrm{op})}$-sequence in $\mathbb{P}^{(\mathrm{op})}$.

Using Lemma 3.6, it is not hard to see that $\varphi^{\omega} \perp_{1}=\bigvee_{i \in \omega} \pi_{i}^{*}\left(\varphi^{i} \perp_{1}\right)$ in the fibration $\underset{\mathbb{C}}{\stackrel{\mathbb{P}}{\downarrow}}$, where $\left(\pi_{i}: F^{\omega} 1 \rightarrow F^{i} 1\right)_{i \in \omega}$ is the limiting cone for the final $F$-sequence in $\mathbb{C}$.

The following is our main result adapted to inductive predicates. In particular, it states that an inductive predicate is computed as a supremum of an $\omega$-chain.

Corollary 5.8. Let $\underset{\mathbb{C}}{\mathbb{P}}$ be a co-well-founded fibration; $F: \mathbb{C} \rightarrow \mathbb{C}$ be a finitary functor; $\varphi$ be a predicate lifting of $F$ along $p$; and $X \stackrel{c}{\rightarrow} F X$ be a coalgebra in $\mathbb{C}$.

1. The $\varphi$-inductive predicate $\llbracket \mu \varphi \rrbracket_{c}$ in $c$ exists. It is obtained by the following reindexing of $\varphi^{\omega} \perp_{1}$, where $c_{\omega}$ is the mediating map in Equation (12).

$$
\llbracket \mu \varphi \rrbracket_{c}=c_{\omega}^{*}\left(\varphi^{\omega} \perp_{1}\right)
$$

2. Moreover, the predicate $\llbracket \mu \varphi \rrbracket_{c}$ is the colimit of the following $\omega$-chain in the fibre $\mathbb{P}_{X}$

$$
\perp_{X} \leqslant\left(c^{*} \circ \varphi\right)\left(\perp_{X}\right) \leqslant\left(c^{*} \circ \varphi\right)^{2}\left(\perp_{X}\right) \leqslant \cdots,
$$

that stabilizes after $\omega$ steps. That is, $\llbracket \mu \varphi \rrbracket_{c}=\bigvee_{i \in \omega}\left(c^{*} \circ \varphi\right)^{i}\left(\perp_{X}\right)$.

Proof. By Lemmas 5.4 and 5.6 and Theorem 3.9.

Corollary 5.9. Let $\varphi$ be a predicate lifting of $F$ along $\underset{\mathbb{C}}{\mathbb{P}}$; and $\begin{gathered}\left(\operatorname{Coalg}\left(\varphi^{(\mathrm{op})}\right)\right)^{(\mathrm{op})} \\ \begin{array}{l}\left.\downarrow p^{(\mathrm{op})}\right)^{(\mathrm{op})} \\ \operatorname{Coalg}(F)\end{array}\end{gathered}$ be the fibrewise

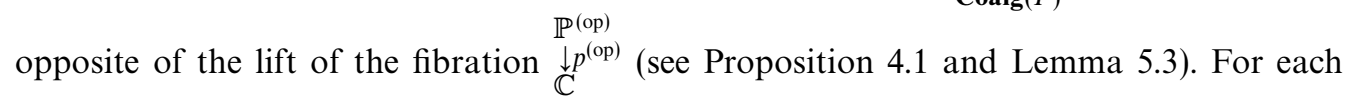
coalgebra $X \stackrel{c}{\rightarrow} F X$, the following diagram commutes:

$$
(\left(\operatorname{Coalg}\left(\varphi^{(\mathrm{op})}\right)\right)^{(\mathrm{op})} \underbrace{}_{\left(\left(\mathbb{P}_{X}\right)^{\mathrm{op}}\right)^{\mathrm{op}}=\mathbb{P}_{X}} \underset{\substack{c \\ \rightarrow}}{\longrightarrow} \operatorname{Alg}\left(c^{*} \circ \varphi\right)
$$

Proof. Apply Proposition 4.2 for the predicate lifting $\varphi^{(\mathrm{op})}$ along $\underset{\mathbb{C}}{\stackrel{\downarrow}{\mathbb{P}^{(\mathrm{op})}}}$, we obtain

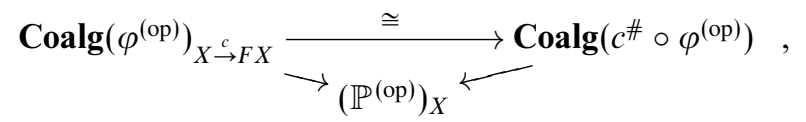

whose opposite categories are the ones in the diagram we want to prove. 
Coalgebra morphisms are compatible with inductive predicates just as in Proposition 3.13. Therefore, the inductive predicates $\llbracket \mu \varphi \rrbracket_{c}$ form a fibrewise initial object $\perp=\llbracket \mu \varphi \rrbracket$ of the fibration ${\overline{p^{(\mathrm{op})}}}^{\text {(op) }}$.

\section{Examples at large}

Here are several results that ensure a fibration to be finitely determined or well-founded, and hence enable us to apply Theorem 3.9. Some of them are well-known; others - especially those which relate fibrations and locally (finitely) presentable categories, including Lemmas 6.3 and 6.7 - seem to be new.

The following results provide sufficient conditions for a fibration to be finitely determined (Definition 3.2). Recall that a full subcategory $\mathbb{F}$ of $\mathbb{P}$ is said to be dense if each object $P \in \mathbb{P}$ is a colimit of the canonical diagram $\mathbb{F} / P \stackrel{\pi}{\rightarrow} \mathbb{F} \hookrightarrow \mathbb{P}$.

Lemma 6.1. Let $\underset{\mathbb{C}}{\mathbb{P}}$ be a fibration with fibrewise limits and colimits and coproducts $\coprod$ between fibres. Assume further that $\mathbb{C}$ is LFP with a set $\mathbb{F}_{\mathbb{C}}$ of FP objects (as in Definition 3.1). If the total category $\mathbb{P}$ has a dense subcategory $\mathbb{F}_{\mathbb{P}}$ such that every $R \in \mathbb{F}_{\mathbb{P}}$ is above $\mathbb{F}_{\mathbb{C}}$ (i.e. $p R \in \mathbb{F}_{\mathbb{C}}$ ), then $p$ is finitely determined.

Proof. The only non-trivial part is the $\Leftarrow$ direction of Cond. 3. For that, it suffices to show that arbitrary $P \in \mathbb{P}$ is a colimit of the diagram $\left(\kappa_{I}^{*} P\right)_{I \in \mathbb{I}}$. Here, $\mathbb{I}$ and $\kappa_{I}$ are as in Cond. 3.

By Lemma C.11, the colimit Colim $\operatorname{leI}_{I \in \mathbb{I}} \kappa_{I}^{*} P$ is described as $\bigvee_{I \in \mathbb{I}} \bigsqcup_{\kappa_{I}} \kappa_{I}^{*} P$ using a sup $\bigvee$ in $\mathbb{P}_{X}$, since $\left(X_{I} \stackrel{\kappa_{I}}{\rightarrow} X\right)_{I \in \mathbb{I}}$ is colimiting. We have $\coprod_{\kappa_{I}} \kappa_{I}^{*} P \leqslant P$ as a counit of an adjunction; therefore, $\operatorname{Colim}_{I \in \mathbb{I}} \kappa_{I}^{*} P \leqslant P$.

Thus, it suffices to show that $P \leqslant \operatorname{Colim}_{I \in \mathbb{I}} \kappa_{I}^{*} P$ in $\mathbb{P}_{X}$. Let $\left(P_{J}\right)_{J \in \mathbb{J}}$ be a diagram in $\mathbb{P}$ such that $P_{J} \in \mathbb{F}_{\mathbb{P}}$ and there is a colimiting cocone $\left(P_{J} \stackrel{g_{J}}{\rightarrow} P\right)_{J \in J}$. Such a diagram exists since $\mathbb{F}_{\mathbb{P}}$ is dense.

By the assumption, for each $J$, the object $P_{J} \in \mathbb{F}_{\mathbb{P}}$ lies above an object in $\mathbb{F}_{\mathbb{C}}$. Therefore, the arrow $p g_{J}: p P_{J} \rightarrow p P=X$ is an object of $\mathbb{F}_{\mathbb{C}} / X$; since $\mathbb{I}=\mathbb{F}_{\mathbb{C}} / X$, we can choose $I_{J} \in \mathbb{I}$ such that $\kappa_{I_{J}}=p g_{J}$. Now an arrow $P_{J} \stackrel{g_{J}}{\rightarrow} P$ in $\mathbb{P}$ induces

$$
P_{J} \leqslant\left(p g_{J}\right)^{*} P=\kappa_{I_{J}}^{*} P
$$

by the universality of Cartesian arrows. We proceed as follows:

$$
P=\operatorname{Colim}_{J \in \mathbb{J}} P_{J} \stackrel{(*)}{=} \bigvee_{J \in \mathbb{J}} \coprod_{p g_{J}} P_{J} \stackrel{(\dagger)}{\leqslant} \bigvee_{J \in \mathbb{J}} \coprod_{\kappa_{I_{J}}} \kappa_{I_{J}}^{*} P \leqslant \bigvee_{I \in \mathbb{I}} \coprod_{\kappa_{I}} \kappa_{I}^{*} P \stackrel{(*)}{=} \underset{I \in \mathbb{I}}{\operatorname{Colim}} \kappa_{I}^{*} P .
$$

For $(*)$, we used Lemma C.11; $(\dagger)$ holds since $I_{J}$ is chosen so that $\kappa_{I_{J}}=p g_{J}$ and Equation (19) hold. This concludes the proof.

Corollary 6.2. Let $\underset{\mathbb{C}}{\mathbb{P}}$ be a fibration with fibrewise limits and colimits and coproducts between fibres, where $\mathbb{C}$ is LFP with a set $\mathbb{F}_{\mathbb{C}}$ of FP objects (in Definition 3.1). If the total category $\mathbb{P}$ is also LFP, with a set $\mathbb{F}_{\mathbb{P}}$ of FP objects (as in Definition 3.1) chosen so that every $R \in \mathbb{F}_{\mathbb{P}}$ is above $\mathbb{F}_{\mathbb{C}}$, then $p$ is finitely determined. 


\subsection{Subobject fibrations}

The following is one of the results that are non-trivial.

Lemma 6.3. Let $\mathbb{C}$ be an LFP category with $\mathbb{F}$ being a set of FP objects (as in Definition 3.1). Then the total category $\operatorname{Sub}(\mathbb{C})$ of the subobject fibration is LFP: the set

$$
\mathbb{F}_{\operatorname{Sub}(\mathbb{C})}:=\{(P \mapsto X) \mid X \in \mathbb{F} \text {, and there exists a strong epi } Z \rightarrow P \text { such that } Z \in \mathbb{F}\}
$$

consists of FP objects in $\operatorname{Sub}(\mathbb{C})$; and every object $(Q \longmapsto Y) \in \operatorname{Sub}(\mathbb{C})$ is a colimit of a filtered diagram in $\mathbb{F}_{\operatorname{Sub}(\mathbb{C})}$.

Proof. The proof is by steps.

Step a. First, we show that $\operatorname{Sub}(\mathbb{C})$ is complete and cocomplete. We rely on Lemma C.11.

$\operatorname{Sub}(\mathbb{C})$

We start with fibrewise limits in $\underset{\mathbb{C}}{\downarrow}$; the proof is like in Jacobs (1999, Example 1.8.3(iii)). By Lemma B.6, an LFP category $\mathbb{C}$ is complete. This equips each fibre $\operatorname{Sub}(X)$ with arbitrary inf's $\bigwedge$ computed as wide pullbacks. A reindexing functor (by pullbacks) preserves these inf's since limits commute. Therefore, by Lemma C.11, the total category $\operatorname{Sub}(\mathbb{C})$ is complete.

Each fibre (which is a poset) has arbitrary inf's; hence, it is a complete lattice and arbitrary sup's also exist.

Next, we show that $\stackrel{\operatorname{Sub}(\mathbb{C})}{\downarrow}$ is a bifibration (Definition C.3). An abstract proof can be given by Freyd's adjoint functor theorem (note that each fibre $\operatorname{Sub}(X)$ is a complete lattice, and that reindexing $f^{*}$ preserves inf's). Instead we explicitly introduce $\coprod$ exploiting a factorization structure of LFP $\mathbb{C}$ (Lemma B.6.2). Namely, given $(P \stackrel{m}{\longmapsto} X) \in \operatorname{Sub}(X)$ and $f: X \rightarrow Y$, the opreindexing $\coprod_{f} P$ is defined by the (StrongEpi, Mono)-factorization of $f \circ m$, as below.

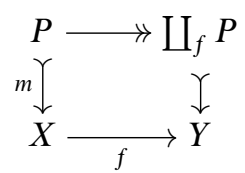

The fact that $\coprod_{f} P \leqslant Q$ if and only if $P \leqslant f^{*} Q$ is easily proved using the diagonalization property of the factorization structure. This establishes $\coprod_{f}$ as a left adjoint to reindexing $f^{*}$. Using Lemma C.11, we conclude that $\operatorname{Sub}(\mathbb{C})$ is cocomplete.

Step b. Let $\mathrm{Im}: \mathbb{C} / Y \rightarrow \operatorname{Sub}(Y)$ be the image functor defined by the (StrongEpi, Mono)factorization (i.e. $\operatorname{Im} f=\coprod_{f} X$ for $f: X \rightarrow Y$ ). In the notation in Lemma B.11.2, we have

$$
\begin{aligned}
\mathbb{F}_{\operatorname{Sub}(\mathbb{C})} & =\{\operatorname{Im} f \mid X \in \mathbb{F}, f \in \mathbb{F} / X\} \\
& =\left\{(P \mapsto X) \in \operatorname{Sub}(\mathbb{C}) \mid X \in \mathbb{F}, P \in \mathbb{F}_{\operatorname{Sub}(X)}\right\} .
\end{aligned}
$$

The set $\mathbb{F}_{\text {Sub }(\mathbb{C})}$ is small, since $\mathbb{F}$ is small and $\mathbb{F}_{\operatorname{Sub}(X)}$ is small for each $X \in \mathbb{F}$.

Step c. First, we prove that $(P \stackrel{m}{\longmapsto} X) \in \mathbb{F}_{\operatorname{Sub}(\mathbb{C})}$ is $\mathrm{FP}$ in $\operatorname{Sub}(\mathbb{C})$.

\footnotetext{
$\sharp$ Opreindexings $\coprod_{f} \dashv f^{*}$ do not have to satisfy the Beck-Chevalley condition.
} 
Sublemma 6.4. Let $\left(Q_{I} \stackrel{n_{I}}{\rightarrow} Y_{I}\right)_{I \in \mathbb{I}}$ be a filtered diagram in $\operatorname{Sub}(\mathbb{C})$. Then pointwise colimits $\operatorname{Colim}_{I \in \mathbb{I}} Y_{I}$ and $\operatorname{Colim}_{I \in \mathbb{I}} Q_{I}$ in $\mathbb{C}$ form a colimit of the diagram in $\operatorname{Sub}(\mathbb{C})$ :

$$
\underset{I \in \mathbb{I}}{\operatorname{Colim}}\left(Q_{I} \stackrel{n_{I}}{\longmapsto} Y_{I}\right)=\left(\underset{I \in \mathbb{I}}{\operatorname{Colim}} Q_{I} \rightarrow \underset{I \in \mathbb{I}}{\operatorname{Colim}} Y_{I}\right)
$$

Proof. (Of the sublemma) On the one hand, by Lemma C.11, the colimit $(Q \stackrel{n}{\mapsto} Y)=$ $\operatorname{Colim}_{I \in \mathbb{I}}\left(Q_{I} \longmapsto Y_{I}\right)$ can be explicitly described as

$$
Y=\underset{I \in \mathbb{I}}{\operatorname{Colim}} Y_{I}, \quad Q=\bigvee_{I \in \mathbb{I}} \coprod_{\kappa_{I}} Q_{I}
$$

where $\left(Y_{I} \stackrel{\kappa_{I}}{\rightarrow} Y\right)_{I \in \mathbb{I}}$ is a colimiting cocone. On the other hand, both $\left(Q_{I}\right)_{I \in \mathbb{I}}$ and $\left(Y_{I}\right)_{I \in \mathbb{I}}$ are $\mathbb{I}$-shaped diagrams in $\mathbb{C}$ with a monotransformation $\left(Q_{I} \stackrel{n_{I}}{\rightarrow} Y_{I}\right)_{I}$. Therefore, by Adámek and Rosický (1994, Corollary 1.60), the induced arrow $n^{\prime}: \operatorname{Colim}_{I} Q_{I} \rightarrow \operatorname{Colim}_{I} Y_{I}$ is monic. In $\operatorname{Sub}(Y)$, we have

$$
\begin{aligned}
Q & =\bigvee_{I \in \mathbb{I}} \operatorname{Im}\left(Q_{I} \stackrel{n_{I}}{\rightarrow} Y_{I} \stackrel{\kappa_{I}}{\rightarrow} Y\right) \\
& =\operatorname{Im}\left(\left(\operatorname{Colim}_{I \in \mathbb{I}} Q_{I}\right) \stackrel{n^{\prime}}{\rightarrow} Y\right) \quad \text { by Lemma B.7 } \\
& =\underset{I \in \mathbb{I}}{\operatorname{Colim}} Q_{I} \text { the arrow } n^{\prime} \text { is already monic. }
\end{aligned}
$$

As we have $Y=\operatorname{Colim}_{I} Y_{I}$ in $\mathbb{C}$, this concludes the proof of the sublemma.

Let $\left(Q_{I} \stackrel{n_{I}}{\longmapsto} Y_{I}\right)_{I \in \mathbb{I}}$ be a filtered diagram in $\operatorname{Sub}(\mathbb{C}) ;(Q \stackrel{n}{\longmapsto} Y)$ be its colimit; and $g:(P \stackrel{m}{\mapsto} X) \rightarrow(Q \stackrel{\text { 品 }}{\mapsto} Y)$ be an arrow in $\operatorname{Sub}(\mathbb{C})$.

There exists an FP object $Z \in \mathbb{F}$ and a strong epimorphism $p: Z \rightarrow P$ by the definition of $\mathbb{F}_{\operatorname{Sub}(\mathbb{C})}$ and $\mathbb{F}_{\operatorname{Sub}(X)}$. The preservation of filtered colimits is shown as follows:

$$
\begin{aligned}
& \underset{I \in \mathbb{I}}{\operatorname{Colim}}\left(\operatorname{Sub}(\mathbb{C})\left((P \stackrel{m}{\longmapsto} X),\left(Q_{I} \stackrel{n_{I}}{\rightarrow} Y_{I}\right)\right)\right) \\
& \stackrel{(*)}{\cong} \operatorname{Colim}_{I \in \mathbb{I}}\left\{\left(f_{I}: X \rightarrow Y_{I}, g_{I}: Z \rightarrow Q_{I}\right) \mid f_{I} \circ m \circ p=n \circ g_{I}\right\} \\
& =\operatorname{Colim}_{I \in \mathbb{I}}\left(\mathbb{C}\left(X, Y_{I}\right) \times_{\mathbb{C}\left(Z, Y_{I}\right)} \mathbb{C}\left(Z, Q_{I}\right)\right) \\
& \quad \text { where } \mathbb{C}\left(X, Y_{I}\right) \times_{\mathbb{C}\left(Z, Y_{I}\right)} \mathbb{C}\left(Z, Q_{I}\right) \text { is a suitable pullback } \\
& \cong\left(\operatorname{Colim}_{I \in \mathbb{I}} \mathbb{C}\left(X, Y_{I}\right)\right) \times_{\operatorname{Colim}_{I \in \mathbb{I}} \mathbb{C}\left(Z, Y_{I}\right)}\left({\underset{I \in \mathbb{I}}{\text { Colim }}}_{\mathbb{C}} \mathbb{C}\left(Z, Q_{I}\right)\right)
\end{aligned}
$$

Sets is LFP and hence filtered colimits and finite limits commute

$\cong \mathbb{C}\left(X, \underset{I \in \mathbb{I}}{\operatorname{Colim}} Y_{I}\right) \times_{\mathbb{C}\left(Z, \operatorname{Colim}_{I \in \mathbb{I}} Y_{I}\right)} \mathbb{C}\left(Z, \underset{I \in \mathbb{I}}{\operatorname{Colim}} Q_{I}\right) \quad X, Z$ are FP in $\mathbb{C}$

$=\mathbb{C}(X, Y) \times_{\mathbb{C}(Z, Y)} \mathbb{C}(Z, Q)$ by Sublemma 6.4

$=\{(f: X \rightarrow Y, g: Z \rightarrow Q) \mid f \circ m \circ p=n \circ g\}$

$\stackrel{(\dagger)}{\cong} \operatorname{Sub}(\mathbb{C})((P \stackrel{m}{\longmapsto} X),(Q \stackrel{n}{\longmapsto} Y))$ 
where the bijection $(*)$ is by the diagonal fill-in

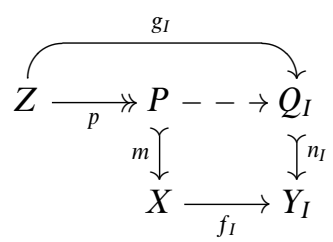

and $(\dagger)$ follows similarly.

Step d. The following observation on canonical diagrams with respect to $\mathbb{F} \subseteq \mathbb{C}$ and $\mathbb{F}_{\operatorname{Sub}(\mathbb{C})} \subseteq \operatorname{Sub}(\mathbb{C})$ is useful.

Sublemma 6.5. The forgetful functor $\begin{gathered}\mathbb{F}_{\mathrm{Sub}(\mathbb{C})} / n \\ \downarrow \\ \mathbb{F} / Y\end{gathered}$ is an opfibration.

Proof. Recall that $\stackrel{\operatorname{Sub}(\mathbb{C})}{\downarrow}$ is a bifibration. Then $\underset{\mathbb{C}}{\stackrel{\mathbb{F}_{\mathrm{Sub}}(\mathbb{C})}{\mathbb{F}}}$ is an opfibration, because the full subcategory $\mathbb{F}_{\operatorname{Sub}(\mathbb{C})} \subseteq \operatorname{Sub}(\mathbb{C})$ is closed under opreindexing as depicted in the diagram

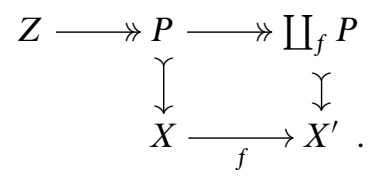

By the diagonal fill-in

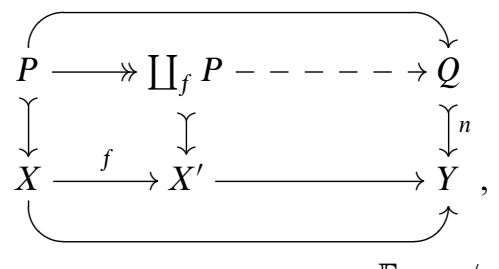

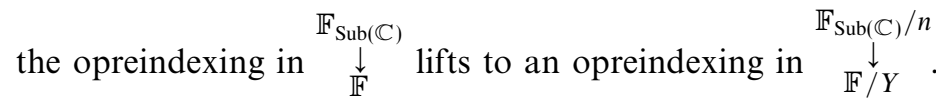

Step e. In the remainder of the proof, we show that every object $(Q \stackrel{n}{\mapsto} Y) \in \operatorname{Sub}(\mathbb{C})$ is a colimit of a filtered diagram in $\mathbb{F}_{\operatorname{Sub}(\mathbb{C})}$. Let us take a filtered diagram $\left(Y_{I}\right)_{I \in \mathbb{I}}$ such that $Y=\operatorname{Colim}_{I \in \mathbb{I}} Y_{I}$ in $\mathbb{C}$ and $Y_{I} \in \mathbb{F}$ (for each $I \in \mathbb{I}$ ).

We shall define a diagram $\left(Q_{J} \stackrel{n_{J}}{\longmapsto} Y_{q J}\right)_{J \in \mathbb{J}}$ in $\mathbb{F}_{\operatorname{Sub}(\mathbb{C})}$ and a functor $q: \mathbb{J} \rightarrow \mathbb{I}$. The (colimiting) cocone $\left(Y_{I} \stackrel{\kappa_{I}}{\rightarrow} Y\right)$ induces a functor $\mathbb{I} \rightarrow \mathbb{F} / Y$, and we obtain an opfibration $\underset{\mathbb{I}}{\mathbb{J}} q$ by change-of-base (Jacobs 1999, Lemma 1.5.1):

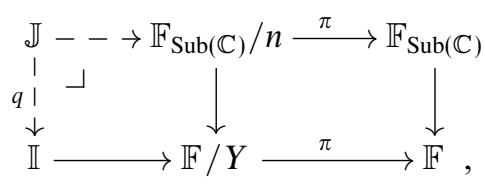


in particular, $\mathbb{J}_{I} \cong\left(\mathbb{F}_{\operatorname{Sub}(\mathbb{C})} / n\right)_{\kappa_{I}} \cong \mathbb{F}_{\operatorname{Sub}\left(Y_{I}\right)} / \kappa_{I}^{*} Q$ :
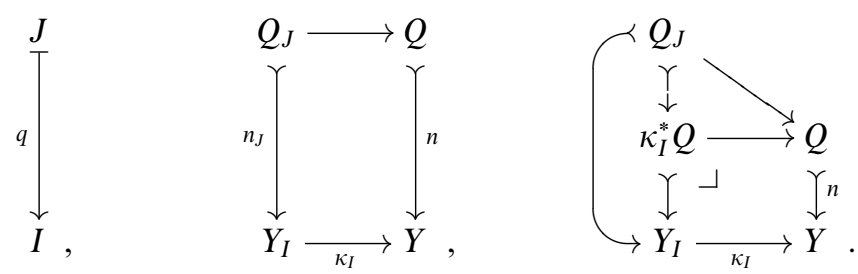

Therefore by Lemma B.11.2, we have a filtered colimit

$$
\bigvee_{J \in \mathbb{J}_{I}} Q_{J}=\kappa_{I}^{*} Q \quad \text { in } \operatorname{Sub}\left(Y_{I}\right)
$$

Moreover, the filtered colimit (22) in $\operatorname{Sub}(X)$ forms a filtered colimit:

$$
\underset{J \in \mathbb{J}_{I}}{\operatorname{Colim}}\left(Q_{J} \longmapsto Y_{I}\right)=\left(\kappa_{I}^{*} Q \longmapsto Y_{I}\right) \quad \text { in } \operatorname{Sub}(\mathbb{C}),
$$

because $\operatorname{Sub}\left(Y_{I}\right) \subseteq \operatorname{Sub}(\mathbb{C})$ is closed under filtered colimits. Consequently,

$$
\begin{aligned}
\underset{J \in \mathbb{J}}{\operatorname{Colim}}\left(Q_{J} \stackrel{n_{J}}{\mapsto} Y_{q J}\right) & \cong \underset{I \in \mathbb{I}}{\operatorname{Colim}} \operatorname{Colim}\left(Q_{J} \mapsto Y_{I}\right) \quad \text { by Lemma C.12 } \\
& =\operatorname{Colim}_{I \in \mathbb{I}}\left(\kappa_{I}^{*} Q \mapsto Y_{I}\right) \quad \text { by Equation }(23) \\
& =\left(\left(\underset{I \in \mathbb{I}}{\operatorname{Colim}} \kappa_{I}^{*} Q\right) \mapsto\left(\underset{I \in \mathbb{I}}{\text { Colim }} Y_{I}\right)\right) \quad \text { by Sublemma } 6.4 \\
& \cong(Q \stackrel{n}{\mapsto} Y) \quad \text { by Lemma B.8. }
\end{aligned}
$$

Step f. Recall that $\underset{\mathbb{I}}{\downarrow} q$ is an opfibration such that the base category $\mathbb{I}$ and each fibre $\mathbb{J}_{I}$ are filtered. It is straightforward to show the total category $\mathbb{J}$ is also filtered.

It follows from Lemma 6.3 and Corollary 6.2 that the internal logic of a topos that is LFP is finitely determined. Note that an (elementary) topos is necessarily a locally Cartesian closed category (LCCC) (see e.g. Jacobs (1999, Proposition 5.4.7)).

Corollary 6.6. Let $\mathbb{C}$ be LFP and at the same time a topos (or more generally an LCCC). Then the subobject fibration $\underset{\mathbb{C}}{\stackrel{\operatorname{Sub}}{\mathbb{C}}}$ is finitely determined.

Proof. By the assumption that $\mathbb{C}$ is an LCCC, $\underset{\mathbb{C}}{\stackrel{\operatorname{Sub}(\mathbb{C})}{\downarrow}}$ has products $\prod_{f} \vdash f^{*}$ between fibres (Jacobs 1999, Corollary 1.9.9). We already proved that each fibre is a complete lattice. These sup's (i.e. colimits in a fibre) are preserved by reindexing $f^{*}$ since the latter is a left adjoint $f^{*} \dashv \prod_{f}$. Namely, the fibration $\underset{\mathbb{C}}{\stackrel{\operatorname{Sub}(\mathbb{C})}{\downarrow}}$ has fibrewise colimits. Opreindexings satisfy the Beck-Chevalley condition since the products $\prod$ do (Jacobs 1999, Lemma 1.9.7).

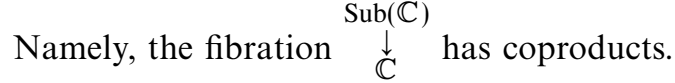




\subsection{Family fibrations}

We turn to the family fibration $\underset{\text { Sets }}{\downarrow} \operatorname{Fam}(\Omega)$

Lemma 6.7. Let $\Omega$ be an algebraic lattice, i.e. a complete lattice in which each element is a join of compact elements. (Equivalently, $\Omega$ is LFP when thought of as a category.) Then the total category $\operatorname{Fam}(\Omega)$ is LFP: The set

$$
\mathbb{F}_{\mathrm{Fam}(\Omega)}:=\{f: X \rightarrow \Omega \mid X \text { is finite, and for each } x \in X, f(x) \text { is compact in } \Omega\}
$$

consists of FP objects in $\operatorname{Fam}(\Omega)$; and every object $(Y, g) \in \operatorname{Fam}(\Omega)$ is a colimit of a filtered diagram in $\mathbb{F}_{\mathrm{Fam}(\Omega)}$. Noting that $f \in \mathbb{F}_{\mathrm{Fam}(\Omega)}$ is above a finite set $X$, by Lemma 6.1, $\operatorname{Fam}(\Omega)$

$\underset{\text { Sets }}{\downarrow}$ is finitely determined.

Proof. Step a. Let us first see that the fibration $\underset{\substack{\downarrow \\ \text { Sets }}}{\operatorname{Fam}(\Omega)}$ has fibrewise limits and colimits and coproducts $\amalg$ between fibres. The former follows from $\Omega$ being a complete lattice; the latter is shown from Jacobs (1999, Lemma 1.9.5). In view of Lemma C.11, it follows that the total category $\operatorname{Fam}(\Omega)$ is cocomplete.

Step b. Before going on, we prove the following.

Sublemma 6.8. Let $\left(Y_{I}\right)_{I \in \mathbb{I}}$ be a filtered diagram in Sets, and $\mathbb{J}=\int Y_{(-)}$be its category of elements, i.e. $\mathbb{J}$ has objects $\left\{\left(I, y^{\prime}\right) \mid I \in \mathbb{I}, y^{\prime} \in Y_{I}\right\}$ and arrows $\mathbb{J}\left(\left(I_{1}, y_{1}^{\prime}\right),\left(I_{2}, y_{2}^{\prime}\right)\right)=$ $\left\{i \in \mathbb{I}\left(I_{1}, I_{2}\right) \mid Y_{i}\left(y_{1}^{\prime}\right)=y_{2}^{\prime}\right\}$. Let $\left(Y_{I} \stackrel{\kappa_{I}}{\rightarrow} Y\right)_{I}$ be a colimiting cocone. For each $y \in Y$, the following full subcategory of $\mathbb{J}$ is filtered:

$$
\mathbb{J}_{y}=\left\{\left(I, y^{\prime}\right) \mid I \in \mathbb{I}, y^{\prime} \in Y_{I}, \kappa_{I}\left(y^{\prime}\right)=y\right\} .
$$

Moreover, the category $\mathbb{J}$ is a disjoint sum of the full subcategories:

$$
\mathbb{J}=\coprod_{y \in \operatorname{Colim}_{I \in \mathbb{I}} Y_{I}} \mathbb{J}_{y} .
$$

Proof. By $Y=\left\{\left(I, y^{\prime}\right) \mid I \in \mathbb{I}, y^{\prime} \in Y_{I}\right\} / \sim$ where $\left(I_{1}, y_{1}^{\prime}\right) \sim\left(I_{2}, y_{2}^{\prime}\right)$ if and only if there exist $I \in \mathbb{I}, i_{1}: I_{1} \rightarrow I$, and $i_{2}: I_{2} \rightarrow I$ such that $Y_{i_{1}}\left(y_{1}^{\prime}\right)=Y_{i_{2}}\left(y_{2}^{\prime}\right)$ (in $Y_{I}$ ).

Step c. We prove that each $(X \stackrel{f}{\rightarrow} \Omega) \in \mathbb{F}_{\mathrm{Fam}(\Omega)}$ is $\mathrm{FP}$ in $\operatorname{Fam}(\Omega)$. Let $\left(\left(Y_{I} \stackrel{g_{I}}{\rightarrow} \Omega\right) \stackrel{\kappa_{I}}{\rightarrow}(Y \stackrel{g}{\rightarrow}\right.$ $\Omega))_{I \in \mathbb{I}}$ be a colimiting cocone in $\operatorname{Fam}(\Omega)$ over a filtered diagram $\mathbb{I}$.

By Lemma C.11, we obtain that $Y=\operatorname{Colim}_{I \in \mathbb{I}} Y_{I}$; and that

$$
\begin{aligned}
g(y) & =\left(\bigvee_{I \in \mathbb{I}} \coprod_{\kappa_{I}} g_{I}\right)(y)=\bigvee_{I \in \mathbb{I}}\left(\left(\coprod_{\kappa_{I}} g_{I}\right)(y)\right) \\
& =\bigvee_{I \in \mathbb{I}}\left(\bigvee_{y^{\prime} \in \kappa_{I}^{-1}(\{y\})} g_{I}\left(y^{\prime}\right)\right)=\bigvee_{\left(I, y^{\prime}\right) \in \mathbb{J}_{y}} g_{I}\left(y^{\prime}\right) \quad \text { for each } y \in Y .
\end{aligned}
$$

The first equality is by Lemma C.11; the second is because the order in the fibre $\operatorname{Fam}(\Omega)_{Y}=\Omega^{Y}$ is pointwise; and the third is by the concrete description (Jacobs 1999, Lemma 1.9.5) of $\coprod$ in $\begin{gathered}\operatorname{Fam}(\Omega) \\ \downarrow \\ \text { Sets }\end{gathered}$. 


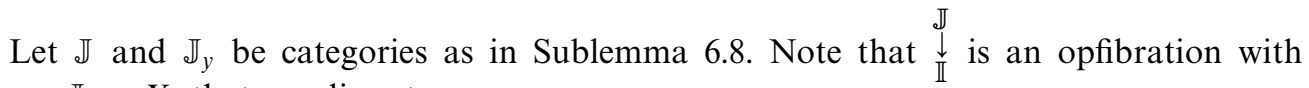
fibres $\mathbb{J}_{I}=Y_{I}$ that are discrete.

$$
\begin{aligned}
& \underset{I \in \mathbb{I}}{\operatorname{Colim}} \operatorname{Fam}(\Omega)\left((X \stackrel{f}{\rightarrow} \Omega),\left(Y_{I} \stackrel{g_{I}}{\rightarrow} \Omega\right)\right) \\
& \cong \underset{I \in \mathbb{I}}{\operatorname{Colim}} \prod_{x \in X} \coprod_{y^{\prime} \in Y_{I}}\left(f(x) \leqslant \Omega g_{I}\left(y^{\prime}\right)\right) \quad \text { by the definition of arrows in } \operatorname{Fam}(\Omega) \\
& \cong \prod_{x \in X} \operatorname{Colim}_{I \in \mathbb{I}} \coprod_{y^{\prime} \in Y_{I}}\left(f(x) \leqslant \Omega g_{I}\left(y^{\prime}\right)\right) \quad \mathbb{I} \text { is filtered and } X \text { is finite } \\
& \cong \prod_{x \in X} \operatorname{Colim}_{\left(I, y^{\prime}\right) \in \mathbb{J}}\left(f(x) \leqslant \Omega g_{I}\left(y^{\prime}\right)\right) \quad \text { by Lemma C.12 } \\
& \cong \prod_{x \in X} \coprod_{y \in \operatorname{Colim}_{I \in \mathbb{I}} Y_{I}} \underset{\left(I, y^{\prime}\right) \in \mathbb{J}_{y}}{\operatorname{Colim}}\left(f(x) \leqslant \Omega g_{I}\left(y^{\prime}\right)\right) \quad \text { by Equation (25) } \\
& \cong \prod_{x \in X} \coprod_{y \in \operatorname{Colim}_{I \in \mathbb{I}} Y_{I}}\left(f(x) \leqslant \Omega \bigvee_{\left(I, y^{\prime}\right) \in \mathbb{J}_{y}} g_{I}\left(y^{\prime}\right)\right) \quad \mathbb{J}_{y} \text { is filtered and } f(x) \in \Omega \text { is compact } \\
& \cong \operatorname{Fam}(\Omega)((X \stackrel{f}{\rightarrow} \Omega),(Y \stackrel{g}{\rightarrow} \Omega)) \text { by Equation }(26),
\end{aligned}
$$

where $\left({ }_{-} \leqslant \Omega_{-}\right)$denotes the homset $\left.\Omega_{(},,_{-}\right)$, which has at most one element, in the lattice $\Omega$ thought of as a category.

Step d. The collection $\mathbb{F}_{\mathrm{Fam}(\Omega)}$ is obviously small.

Step e. We are done if we prove that every object $P \in \operatorname{Fam}(\Omega)$ is a filtered colimit of its subobjects from $\mathbb{F}_{\mathrm{Fam}(\Omega)}$. This easily follows from the fact that the same is true in Sets (obvious) and in $\Omega$ (being an algebraic lattice).

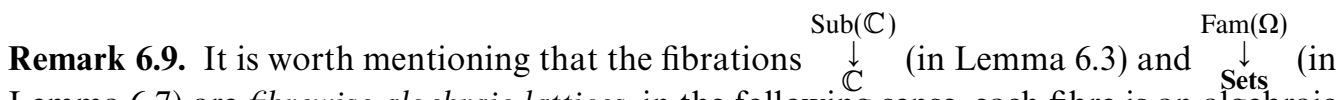
Lemma 6.7) are fibrewise algebraic lattices, in the following sense, each fibre is an algebraic lattice; and each reindexing $f^{*}$ between fibres is a 'homomorphism' of algebraic lattices, which we define to be a monotone map that preserves arbitrary meets and directed joins. In other words, each reindexing $f^{*}$ is a finitary right adjoint functor. We have essentially shown this fact in the proofs for these examples (Lemmas 6.3 and 6.7). Indeed, through the Gabriel-Ulmer duality (Gabriel and Ulmer 1971), a finitary right adjoint functor $f^{*}: \mathbb{P}_{Y} \rightarrow \mathbb{P}_{X}$ between LFP categories corresponds to a functor $\coprod_{f}:\left(\mathbb{P}_{X}\right)_{\mathrm{FP}} \rightarrow\left(\mathbb{P}_{Y}\right)_{\mathrm{FP}}$ that preserves finite colimits, where $\left({ }_{-}\right)_{F P}$ denotes the full subcategory consisting of all the FP objects. All this indicates that the preservation of compact elements under the coproduct $\coprod$ is crucial in our developments.

We shall, however, assume Cond. 2, the stronger condition that reindexing arrows $f^{*}$ preserve arbitrary joins, too. This simplifies definitions and emphasizes duality as in Lemma 5.6.

\subsection{Presheaf categories}

Presheaf categories are well-known examples of LFP categories. See Adámek and Rosický (1994). 
Example 6.10 (Presheaf categories). Let $\mathbb{A}$ be small. The presheaf category Sets $^{\mathbb{A}}$ is LFP: the set $\mathbb{F}$ of finite colimits of representable presheaves $\mathbf{y} A$, where $\mathbf{y} A=\mathbb{A}\left(A\right.$, $\left._{-}\right)$, satisfies the conditions of Definition 3.1. Indeed, any presheaf $X$ is a filtered colimit of objects in $\mathbb{F}$ since $X$ is a colimit (that is not necessarily filtered) of representable presheaves (Lemma 6.14).

For the subobject fibration of a presheaf category Sets $^{\mathbb{A}}$, Cond. 3.3 and $\overline{3.3}$ in Definition 3.3 (for $X \in \mathbb{F}$ ) reduce to the representable case $X=\mathbf{y} A$.

Lemma 6.11. The subobject fibration $\begin{gathered}\operatorname{Sub}\left(\operatorname{Sets}^{\mathbb{A}}\right) \\ \downarrow\end{gathered}$ is well-founded if and only if for all $A \in \mathbb{A}$ the poset $\operatorname{Sub}(\mathbf{y} A)$ has no strictly descending chain. The subobject fibration is co-well-founded if and only if for all $A \in \mathbb{A}$ the poset $\operatorname{Sub}(\mathbf{y} A)$ has no strictly ascending chain.

Sublemma 6.12. Let $\left(X_{I}\right)_{I}$ be a finite diagram in $\operatorname{Sets}^{\mathbb{A}}$. If for each $I$ the poset $\operatorname{Sub}\left(X_{I}\right)$ has no strictly descending chain, then so does $\operatorname{Sub}\left(\operatorname{Colim}_{I} X_{I}\right)$. If for each $I$ the poset $\operatorname{Sub}\left(X_{I}\right)$ has no strictly ascending chain, then so does $\operatorname{Sub}\left(\operatorname{Colim}_{I} X_{I}\right)$.

Proof. (Of Sublemma 6.12) We rely on a presentation of colimits by coproducts and coequalizers. In a topos (hence a regular category) Sets $^{\mathbb{A}}$ coproducts are disjoint (see e.g. Jacobs (1999, Exercise 4.5.1)); thus, we have an isomorphism of posets

$$
\operatorname{Sub}\left(X_{1}+\cdots+X_{n}\right) \cong \operatorname{Sub}\left(X_{1}\right) \times \cdots \times \operatorname{Sub}\left(X_{n}\right) .
$$

Let $X \rightrightarrows Y \stackrel{e}{\rightarrow} Z$ be a coequalizer in $\operatorname{Sets}^{\mathbb{A}}$. The correspondence $e^{*}: \operatorname{Sub}(Z) \rightarrow \operatorname{Sub}(Y)$ is easily seen to be injective. Indeed, assume $P \neq P^{\prime}$ in $\operatorname{Sub}(Z)$; then $P A \neq P^{\prime} A$ in Sets for some $A \in \mathbb{A}$, and since $e_{A}: Y A \rightarrow Z A$ is surjective, we have

$$
\left(e^{*} P\right) A=e_{A}^{-1}(P A) \neq e_{A}^{-1}\left(P^{\prime} A\right)=\left(e^{*} P\right) A .
$$

Therefore, if $\operatorname{Sub}(Z)$ has a strictly descending or ascending chain, $\operatorname{Sub}(Y)$ has a strictly descending or ascending chain, respectively. This concludes the proof of the sublemma.

Proof. (Of Lemma 6.11) By Example 6.10, Corollary 6.6 and Sublemma 6.12.

The previous lemma reduces the size problem of the fibration $\underset{\downarrow}{\operatorname{Sub}\left(\mathbf{S e t s}^{\mathbb{A}}\right)}$ to that of $\operatorname{Sub}(\mathbf{y} A)$. In calculating $\operatorname{Sub}(\mathbf{y} A)$, we will be using the following well-known characterization of presheaves as colimits of representables.

Definition 6.13. Let $\mathbb{A}$ be a small category and $P: \mathbb{A} \rightarrow$ Sets be a functor. The category of elements of $P$, which is denoted by $\int P$, consists of objects that are pairs $(A \in \mathbb{A}, p \in P A)$ and arrows

$$
\left(\int P\right)((A, p),(B, q))=\{f: A \rightarrow B \mid P(f)(p)=q\}
$$

Lemma 6.14. Any presheaf $P \in$ Sets $^{\mathbb{A}}$ is canonically isomorphic to the colimit of representable functors indexed by the category of elements: $P \cong \operatorname{Colim}_{(A, p) \in \int P} \mathbf{y} A$. 
Proof. For each object $(A, p) \in \int P$, an arrow $\mathbf{y} A \rightarrow P$ is induced by $(\mathbf{y} A) B=\mathbb{A}(A, B) \ni$ $g \mapsto P(g)(p) \in P B$. It is not difficult to see that these arrows $\mathbf{y} A \rightarrow P$ are natural in $(A, p) \in \int P$ and form a colimiting cocone. See e.g. Adámek and Rosický (1994, Proposition 1.45) for details.

Proposition 6.16 (presented later) will be our principal tool for calculating $\operatorname{Sub}(\mathbf{y} A)$. The proposition is inspired by the following cocompletion results (Lemma 6.15), which will not be themselves used in our subsequent technical developments.

Lemma 6.15. Let $\mathbb{A}$ be a small category.

1. The category Sets ${ }^{\mathbb{A}}$ of presheaves is a free cocompletion of the category $\mathbb{A}^{\text {op }}$ (with the unit $\mathbf{y}: \mathbb{A}^{\text {op }} \rightarrow$ Sets $^{\mathbb{A}}$ ), that is, for a functor $F: \mathbb{A}^{\text {op }} \rightarrow \mathbb{C}$ to a cocomplete category $\mathbb{C}$ there uniquely (up to natural isomorphisms) exists a cocontinuous functor $G:$ Sets $^{\mathbb{A}} \rightarrow \mathbb{C}$ such that $F \cong G \circ \mathbf{y}$.

2. Let $P \in$ Sets $^{\mathbb{A}}$ be a presheaf. There exists an equivalence of categories $\operatorname{Sets}^{\mathbb{A}} / P \cong$ $\operatorname{Sets}^{\int} P$. Hence, the slice category $\operatorname{Sets}^{\mathbb{A}} / P$ is a free cocompletion of the category $\left(\int P\right)^{\mathrm{op}}$.

3. Let $A \in \mathbb{A}$ be an object. The category $\operatorname{Sets}^{\mathbb{A}} /(\mathbf{y} A)$ is equivalent to the category Sets $^{A / \mathbb{A}}$. Hence, the slice category Sets ${ }^{\mathbb{A}} /(\mathbf{y} A)$ is a free cocompletion of the category $(A / \mathbb{A})^{\mathrm{op}}=\mathbb{A}^{\mathrm{op}} / A$,

Proof. The item 1 is well-known: the functor $G$ is given by $G P=\operatorname{Colim}_{(A, p) \in \int P} F A$. In particular, when we take $\mathbf{y}: \mathbb{A}^{\text {op }} \rightarrow$ Sets $^{\mathbb{A}}$ as $F$, we obtain $G$ that is naturally isomorphic to id : Sets $^{\mathbb{A}} \rightarrow$ Sets $^{\mathbb{A}}$. This generalizes Lemma 6.14.

The item 2 - with a strong fibrational flavour, via the Grothendieck construction - is found e.g. in Mac Lane and Moerdijk (1992, Exercise III.8.(a)). The equivalence is given explicitly by

$$
\begin{array}{lrl}
\text { Sets }^{\mathbb{A}} / P & \text { Sets }^{\int P} & \text { Sets }^{\int P} \longrightarrow \text { Sets }^{\mathbb{A}} / P \\
(Q \stackrel{\alpha}{\longrightarrow} P) \longmapsto\left[(A, p) \longmapsto\left(\alpha_{A}\right)^{-1}(\{p\})\right] & R \longmapsto\left[A \longmapsto \coprod_{p \in P A} R(A, p)\right],
\end{array}
$$

where, in the last entry, we only presented a presheaf in $\operatorname{Sets}^{\mathbb{A}}$ (an arrow to $P$ is given obviously by a projection).

The item 3 is obtained from the item 2 and the fact that $\int(\mathbf{y} A)=A / \mathbb{A}$ (an easy observation).

\section{Proposition 6.16.}

1. Let $\mathbb{A}$ be small. For any $A \in \mathbb{A}$, the subset

$$
\{\operatorname{Im}(\mathbf{y} B \stackrel{\mathbf{y} f}{\longrightarrow} \mathbf{y} A) \mid B \in \mathbb{A}, f: A \rightarrow B\} \subseteq \operatorname{Sub}(\mathbf{y} A)
$$

is dense as a full subcategory, that is, for any subpresheaf $Q \mapsto \mathbf{y} A$, there canonically exists a family $\left(f_{I}: A \rightarrow B_{I}\right)_{I}$ such that $Q=\bigvee_{I} \operatorname{Im}\left(\mathbf{y} f_{I}\right)$. Here, $\operatorname{Im}(\alpha)$ denotes the image of an arrow $\alpha$.

2. Furthermore, assume that every arrow $f$ with domain $A \in \mathbb{A}$ factors as $f=m \circ e$ with an epi $e$ and a split mono $m$. Then (the image of) the canonical embedding 
$\operatorname{Quot}(A) \longmapsto \operatorname{Sub}(\mathbf{y} A)$ is dense. Here, Quot $(A)$ denotes the poset of quotient objects of A.

Proof. A detailed proof is given in Appendix D.

Corollary 6.17. If the following condition 1 holds for each $A \in \mathbb{A}$, then the fibration $\operatorname{Sub}\left(\operatorname{Sets}^{\mathbb{A}}\right)$

$\underset{\text { Sets }^{\mathbb{A}}}{\downarrow}$ is both well-founded and co-well-founded.

1. The subset $\{\operatorname{Im}(\mathbf{y} f) \mid B \in \mathbb{A}, f: B \rightarrow A\} \subseteq \operatorname{Sub}(\mathbf{y} A)$ is finite. Furthermore, for each $A \in \mathbb{A}$, the following condition 2 implies the condition 1 above.

2. Any arrow $f$ with domain $A$ factors as $f=m \circ e$ with an epi $e$ and a split mono $m$, and moreover, $\operatorname{Quot}(A)$ is a finite set.

Proof. By Lemma 6.11, it is enough to show that for each $A \in \mathbb{A}$ the poset $\operatorname{Sub}(\mathbf{y} A)$ is finite.

Assume that $A \in \mathbb{A}$ satisfies the condition 1: the subset $\{\operatorname{Im}(\mathbf{y} f) \mid B \in \mathbb{A}, f: B \rightarrow$ $A\} \subseteq \operatorname{Sub}(\mathbf{y} A)$ is finite. By Proposition 6.16.1, we have $\operatorname{Sub}(\mathbf{y} A)=\left\{\bigvee_{I} \operatorname{Im}\left(\mathbf{y} f_{I}\right) \mid\left(B_{I} \in \mathbb{A}\right.\right.$, $\left.\left.f_{I}: B \rightarrow A\right)_{I}\right\}$, which is also finite.

That the condition 2 implies 1 follows from Proposition 6.16.2.

To determine whether $\operatorname{Im}(\mathbf{y} f)=\operatorname{Im}(\mathbf{y} g)$ holds for arrows $f$ and $g$ with the same domain, the following lemma is useful.

Lemma 6.18. The inclusion relation $\leqslant$ on $\{\operatorname{Im}(\mathbf{y} f) \in \operatorname{Sub}(\mathbf{y} A) \mid B \in \mathbb{A}, f: A \rightarrow B\}$ is the partial order induced by the preorder $\lesssim$ on $\{f \mid B \in \mathbb{A}, f: A \rightarrow B\}$. The latter is defined by

$$
(f: A \rightarrow B) \lesssim(g: A \rightarrow C) \text { if and only if } f=h \circ g \text { for some } h: C \rightarrow B \text {. }
$$

Proof. Let $f: A \rightarrow B, g: A \rightarrow C$ be arrows in $\mathbb{A}$. We first observe that

$$
\begin{aligned}
(\operatorname{Im}(\mathbf{y} f)) D & =\operatorname{Im}\left((\mathbf{y} B) D \stackrel{(\mathbf{y} f)_{D}}{\longrightarrow}(\mathbf{y} A) D\right) \\
& =\left\{(\mathbf{y} f)_{D}(k) \mid k \in(\mathbf{y} B) D\right\} \\
& =\{k \circ f: A \rightarrow D \mid k: B \rightarrow D\}
\end{aligned}
$$

for $D \in \mathbb{A}$.

Assume that $\operatorname{Im}(\mathbf{y} f) \leqslant \operatorname{Im}(\mathbf{y} g)$ in $\operatorname{Sub}(\mathbf{y} A)$. In particular, it holds $(\operatorname{Im}(\mathbf{y} f)) B \subseteq(\operatorname{Im}(\mathbf{y} g)) B$ as subsets of $(\mathbf{y} A) B=\mathbb{A}(A, B)$. We have $f=\operatorname{id}_{B} \circ f \in(\operatorname{Im}(\mathbf{y} f)) B$ by Equation (27); hence, $f \in(\operatorname{Im}(\mathbf{y} g)) B$. Thus, there exists $h: C \rightarrow B$ such that $f=h \circ g$, which is the definition of $f \lesssim g$.

Conversely, assume that $f=h \circ g$ for some $h: C \rightarrow B$. For any $D \in \mathbb{A}$, we have

$$
\begin{aligned}
(\operatorname{Im}(\mathbf{y} f)) D & =\{k \circ h \circ g: A \rightarrow D \mid k: B \rightarrow D\} \quad \text { by Equation (27) } \\
& \subseteq\left\{k^{\prime} \circ g: A \rightarrow D \mid k^{\prime}: C \rightarrow D\right\} \\
& =(\operatorname{Im}(\mathbf{y} g)) D \quad \text { by Equation }(27)
\end{aligned}
$$

as subsets of $(\mathbf{y} A) D$. Therefore, $\operatorname{Im}(\mathbf{y} f) \leqslant \operatorname{Im}(\mathbf{y} g)$. 


\section{Concrete examples}

Example 7.1 (Pred). The fibration $\stackrel{\substack{\text { Pred } \\ \downarrow}}{\downarrow}$ for the conventional setting of classical logic is easily seen to be well-founded and co-well-founded. In particular, Pred $_{X} \cong \mathcal{P} X$ is finite if $X$ is FP (i.e. finite). Therefore, to any finitary $F$ and any predicate lifting $\varphi$, the results in Section 3 apply.

The (interpretations of the) formulas in $\mathrm{R} v$ (see Example 3.10) are examples of coin-

ductive predicates in $\begin{gathered}\text { Pred } \\ \text { Sets }\end{gathered}$. Besides them, the study of coalgebraic modal logic has identified many predicate liftings for many functors $F$ (probabilistic systems, neighbourhood frames, strategy frames, weighted systems, etc.; see e.g. Cîrstea et al. (2011) and the references therein). These 'modalities' all define coinductive predicates, to which the results in Section 3 may apply.

Example 7.2 (Rel). The fibration $\underset{\substack{\downarrow \\ \downarrow}}{\text { Rel }}$ can be introduced from $\stackrel{\text { Pred }}{\downarrow}$ via change-of-base; concretely, an object of $\operatorname{Rel}$ is a pair $(X, R)$ of a set $X$ and a relation $R \subseteq X \times X$; an arrow $f:(X, R) \rightarrow(Y, S)$ is a function $f: X \rightarrow Y$ such that $x R x^{\prime}$ implies $f(x) S f\left(x^{\prime}\right)$. See Jacobs (1999, p. 14).

This fibration, similarly to $\begin{gathered}\text { Pred } \\ \text { Sets }\end{gathered}$, is easily seen to be well-founded and co-well-founded; therefore, to any finitary $F$, the results in Section 3 apply. A predicate lifting $\varphi$ along Rel

$\underset{\downarrow}{\downarrow}$ is more commonly called a relation lifting (Hermida and Jacobs 1998); by choosing suitable $\varphi$ for given $F$ (a 'sufficiently comprehensive' one) like in Hermida and Jacobs (1998), a $\varphi$-invariant is precisely an $F$-bisimulation relation (in the coalgebraic sense), and the $\varphi$-coinductive predicate is $F$-bisimilarity. We expect that the $\omega$-behavioural bound in Theorem 3.9 can be used to bound execution of bisimilarity checking algorithms by partition refinement (for many different functors $F$ ).

In the following example, one can think of $\Omega$ as a Heyting algebra, and then the underlying logic becomes constructive.

Example 7.3 $(\operatorname{Fam}(\Omega))$. Let $\Omega$ be an algebraic lattice that has no strictly descending $\left(\omega^{\mathrm{op}}\right.$ )chains. Then the family fibration $\underset{\operatorname{Fam}(\Omega)}{\downarrow}$ Sets is well-founded (see Lemma 6.7). Therefore, to any finitary $F$, the results in Section 3 apply. It is not hard to interpret the language $R v$ in this setting, by defining predicate liftings similar to Equation (7). This gives examples $\operatorname{Fam}(\Omega)$ of coinductive predicates in $\underset{\substack{\downarrow \\ \text { Sets }}}{\downarrow}$.

Similarly, fibrations $\underset{\text { Sets }}{\operatorname{Fam}\left(\Omega^{\text {op }}\right)}$ are co-well-founded for algebraic lattices $\Omega$ by Lemma 5.6, because the fibrations are fibrewise opposite of well-founded fibrations $\underset{\substack{\operatorname{Fam}(\Omega) \\ \text { Sets }}}{\downarrow}$.

\subsection{Presheaf examples}

Let $\mathbf{F}$ be the category of natural numbers as finite sets (i.e. $n=\{0,1, \ldots, n-1\}$ ) and all functions between them; $\mathbf{F}_{+}$be its full subcategory of nonzero natural numbers; and $\mathbf{I}$ 
be the category of natural numbers and injective functions. Coalgebras in the presheaf categories Sets ${ }^{\mathbf{F}}$, Sets $^{\mathbf{F}}+$ and Sets ${ }^{\mathbf{I}}$ are commonly used for modelling processes in various name-passing calculi. For the $\pi$-calculus, Sets $^{\mathbf{I}}$ has been found appropriate (see e.g. Stark (1996), Fiore and Turi (2001) and Fiore and Staton (2006)); while for the fusion calculus, we do need non-injective functions in $\mathbf{F}$ or $\mathbf{F}_{+}$(see Miculan (2008) and Staton (2011)).

Inspired by Klin (2007), we are interested in coinductive predicates for such processes. They are naturally modelled in the subobject fibration of a presheaf category. Here, we find a distinction: the subobject fibrations of both $\mathbf{S e t s}^{\mathbf{F}}$ and $\mathbf{S e t s}^{\mathbf{F}}+$ are well-founded and co-well-founded; but that of Sets ${ }^{\mathbf{I}}$ is not well-founded (it is co-well-founded). In view of Lemma 6.11 , the only condition to check is Cond. 3.3 or $\overline{3.3}$ for $X=\mathbf{y} A$.

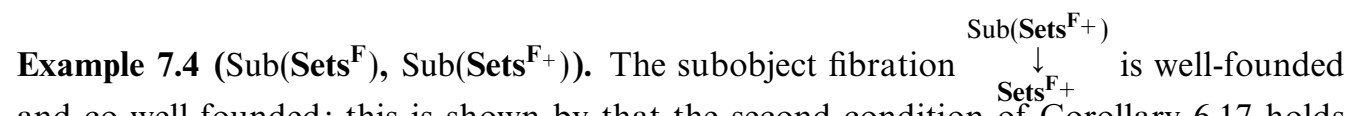
and co-well-founded: this is shown by that the second condition of Corollary 6.17 holds for any $A \in \mathbf{F}_{+}$. An important fact here is that in $\mathbf{F}$ (or in Sets) a mono with a non-empty domain splits, and thus every mono in $\mathbf{F}_{+}$is a split mono.

The subobject fibration $\begin{gathered}\operatorname{Sub}\left(\mathbf{S e t s}^{\mathbf{F}}\right) \\ \downarrow \\ \text { Sets }^{\mathbf{F}}\end{gathered}$ is well-founded and co-well-founded, too. To show that $\operatorname{Sub}(\mathbf{y} 0)$ is finite, we appeal directly to the first condition of Corollary 6.17: We observe by Lemma 6.18 that the set $\{\operatorname{Im}(\mathbf{y} f) \mid n \in \mathbf{F}, f: 0 \rightarrow n\}$ is equal to the two-element set $\left\{\operatorname{Im}\left(\mathbf{y}\left(0 \stackrel{\mathrm{id}_{0}}{\rightarrow} 0\right)\right), \operatorname{Im}(\mathbf{y}(0 \stackrel{!}{\rightarrow} 1))\right\}$ since $0 \stackrel{!}{\rightarrow} n$ and $0 \stackrel{!}{\rightarrow} m$ factor through each other, for each $n, m \geqslant 1$.

We turn to functors $F$ and $\varphi$. In modelling processes of name-passing calculi as coalgebras in these categories, one typically uses endofunctors $F$ that are constructed from the following building blocks. Let $\mathbf{N} \in\left\{\mathbf{F}, \mathbf{F}_{+}, \mathbf{I}\right\}$.

- Constant functors, binary sum + , binary product $\times$ and exponentials $\left(_{-}\right)^{X}$. These are much like for polynomial functors on Sets. An important example of the first is the name presheaf $\mathcal{N}=\operatorname{Hom}\left(1,{ }_{-}\right) \in \operatorname{Sets}^{\mathbf{N}}$.

- The abstraction functor $\delta:$ Sets $^{\mathbf{N}} \rightarrow$ Sets $^{\mathbf{N}}$ given by $\delta X=X\left({ }_{-}+1\right)$.

- The free semilattice functor $\mathcal{P}_{\mathrm{f}}$ for finite branching. This captures Kuratowski finiteness and suitable in Sets ${ }^{I}$. See e.g. Fiore and Turi (2001) and Staton (2011).

- In Sets ${ }^{\mathbf{F}}$ and $\mathbf{S e t s}^{\mathbf{F}_{+}}$, another choice of a 'finite powerset functor' $\widetilde{K}$ is more appropriate. See Miculan (2008); also, Staton (2011, p. 4).

All such functors are known to be finitary (see e.g. Miculan (2008)).

Coinductive predicates in this setting can be introduced much like $\mathrm{R} v$ in Example 2.4 (note that Sets $^{\mathbf{N}}$ is a topos for $\mathbf{N} \in\left\{\mathbf{F}, \mathbf{F}_{+}, \mathbf{I}\right\}$ ), for properties like deadlock freedom. Such a language can be extended further through the modalities proposed in Klin (2007): they correspond to constructions specific to presheaves and include the modality $\langle\bar{a}(b)\rangle$ for a binding 'input' operation. More examples will be worked out in our future paper.

Example $7.5\left(\operatorname{Sub}\left(\operatorname{Sets}^{\omega}\right), \operatorname{Sub}\left(\operatorname{Sets}^{\mathbf{I}}\right)\right)$. Consider the presheaf category Sets $^{\omega}$ over the $\operatorname{Sub}\left(\right.$ Sets $\left.^{\omega}\right)$

ordinal $\omega$ as a poset. The fibration $\underset{\text { Sets }}{ }{ }^{\omega}$ is finitely determined but not well-founded. It fails to satisfy Cond. 3.3 in Definition 3.3 : Let $P_{n}: \omega \rightarrow$ Sets be the family of presheaves 
defined by

$$
P_{n}(m):=\quad(0 \text { if } m<n ; \quad 1 \text { if } n \leqslant m)
$$

for each $n \in \omega$. Then $P_{0}>P_{1}>\cdots$ is a strictly descending chain in $\operatorname{Sub}(\mathbf{y} 0)$. The same counterexample works for $\operatorname{Sub}\left(\operatorname{Sets}^{\mathbf{I}}\right)$.

In contrast, the fibrations $\begin{gathered}\operatorname{Sub}\left(\mathbf{S e t s}^{\omega}\right) \\ \downarrow \\ \text { Sets }^{\omega}\end{gathered}$ and $\begin{gathered}\operatorname{Sub}\left(\mathbf{S e t s}^{\mathbf{I}}\right) \\ \downarrow \\ \text { Sets }^{\mathbf{I}}\end{gathered}$ are co-well-founded, by Lemma 6.11 and the following lemma.

Lemma 7.6. For $\mathbb{A} \in\{\omega, \mathbf{I}\}$ and for any $n \in \mathbb{A}$, the poset $\operatorname{Sub}(\mathbf{y} n)$ is isomorphic to the opposite of the ordinal $\omega+1=\omega \cup\{\omega\}$. Hence, $\operatorname{Sub}(\mathbf{y} n)$ has no strictly increasing chain.

Proof. First, we shall invoke Lemma 6.18. Let $f: n \rightarrow m$ be an arrow in $\mathbb{A}$. Note that the existence of the arrow $f$ induces $m \geqslant n$ as natural numbers. For an arrow $g: n \rightarrow m^{\prime}$ in $\mathbb{A}$, it is easy to see that $f$ factors through $g$ if and only if $m^{\prime} \leqslant m$. In particular, arrows $f, f^{\prime}: n \rightrightarrows m$ factor through each other; therefore, we may denote by $\operatorname{Im}(\mathbf{y} m)$ the image $\operatorname{Im}(\mathbf{y} m \stackrel{\mathbf{y} f}{\rightarrow} \mathbf{y} n) \in \operatorname{Sub}(\mathbf{y} n)$. Moreover, by Lemma 6.18, we have

$$
\operatorname{Im}(\mathbf{y} m) \leqslant \operatorname{Im}\left(\mathbf{y} m^{\prime}\right) \text { if and only if } m \geqslant m^{\prime} .
$$

Therefore, there exists an isomorphism of posets

$$
I: \omega^{\mathrm{op}} \stackrel{\cong}{\longrightarrow}\{\operatorname{Im}(\mathbf{y} m) \mid m \geqslant n\}=\{\operatorname{Im}(\mathbf{y} m \stackrel{\mathbf{y} f}{\rightarrow} \mathbf{y} n) \in \operatorname{Sub}(\mathbf{y} n) \mid m \in \mathbb{A}, f: n \rightarrow m\}
$$

defined by $I(k)=\operatorname{Im}(\mathbf{y}(n+k))$.

We shall induce an isomorphism (the monotone function $J$ below) between the 'cocompletion' of both-hand sides of the isomorphism $I$. Let $\operatorname{DSub}\left(\omega^{\text {op }}\right)$ be the poset of downward closed subsets of $\omega^{\text {op }}$ ordered by inclusion, and $\omega+1$ be the ordinal. Let $h:(\omega+1)^{\mathrm{op}} \rightarrow \operatorname{DSub}\left(\omega^{\mathrm{op}}\right)$ be a function such that

$$
h\left(k^{\prime}\right)=\left(\downarrow k \text { if } k^{\prime}=k \in \omega ; \quad \varnothing \text { if } k^{\prime}=\omega\right)
$$

for $k^{\prime} \in \omega+1$, where $\downarrow k=\{k, k+1, \ldots\}$ is the downward closure of $\{k\} \subseteq \omega^{\text {op }}$. It is easy to see that $h$ becomes an isomorphism of posets. Since the poset $\operatorname{Sub}(\mathbf{y} n)$ is cocomplete, the isomorphism $I$ induces the diagram

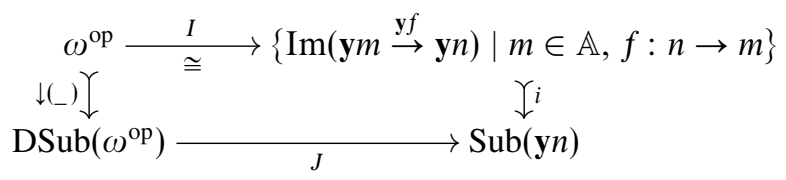

in Posets, where $i$ is the canonical inclusion, and $J(S)=\bigvee_{k \in S} I(k)$ is the sup of the images under the isomorphism. It is enough to show that $J$ is also an isomorphism of posets.

On the one hand, the inclusion $i$ is dense as a full subcategory by Proposition 6.16.1, that is, the monotone function $J$ is surjective. On the other hand, the nullary sup $J(\varnothing)=0$ in $\operatorname{Sub}(\mathbf{y} n)$ is strictly less than any other image $J(\downarrow k)=I(k)=\operatorname{Im}(\mathbf{y}(n+k))$ for $k \in \omega$. Hence, the monotone function $J: \operatorname{DSub}\left(\omega^{\text {op }}\right) \rightarrow \operatorname{Sub}(\mathbf{y} n)$ is an embedding (i.e. a monotone injection 
that reflects the order) that extends the embedding $i \circ I: \omega^{\text {op }} \rightarrow \operatorname{Sub}(\mathbf{y} n)$. Therefore, the monotone function $J$ is a surjective embedding, that is, an isomorphism of posets.

In contrast to Sets $^{\omega}$, the subobject fibration for Sets ${ }^{\omega^{\mathrm{op}}}$ is well-founded and co-wellfounded by Corollary 6.17. Indeed, arrows $f: n \rightarrow m$ in $\omega^{\text {op }}$ has an (Epi, SplitMono)factorization $n \rightarrow m \mapsto m$, and $\operatorname{Quot}_{\omega^{\text {op }}}(n)=\{n, n-1, \ldots, 0\}$ is a finite set.

Remark 7.7. Well-foundedness fails in $\operatorname{Sub}\left(\operatorname{Sets}^{\omega}\right)$, Sub(Sets $\left.{ }^{\mathbf{I}}\right)$ and in $\operatorname{Fam}(\Omega)$ for $\Omega$ that does have a strictly descending $\omega^{\mathrm{op}}$-chain. This means the logics modelled by the fibrations are inherently 'big.' Still, extensions of our results in Section 3 are possible from finitary (i.e. $\omega$-presentable) to the $\lambda$-presentable setting for bigger $\lambda$, so that they apply to the (current) non-examples.

\section{Conclusions and future work}

We have investigated a mathematical theory of coinductive (and inductive) predicates over coinductive datatypes, formalized categorically using coalgebras and fibrations. Our technical results are about iterative constructions of coinductive predicates; they are stated also in abstract categorical terms, using the language of locally presentable categories.

In this paper, we focussed on purely coinductive predicates and purely inductive ones. However, in system verification, their combination is very commonly used. Such mixture of induction and coinduction is studied fibrationally in Hensel and Jacobs (1997), but over mixed inductive and coinductive data types, and not over a coalgebra. We believe a recent lattice-theoretic characterization of nested/alternating least and greatest fixed points (Hasuo et al. 2016) will provide a handle for suitably extending the current work.

Search for useful coinduction proof principles is an active research topic (see e.g. Bonchi and Pous (2013) and Hur et al. (2013)). We are interested in the questions of whether these principles are sound in a general fibrational setting, and what novel proof principles a fibrational view can lead to. In fact, the well-known technique of coinduction up-to has been formulated in fibrational terms (Bonchi et al. 2014) and revealed exciting new applications like nominal automata.

Coalgebraic modal logic is more and more often introduced based on a Stone-like duality (see e.g. Klin (2007)). Fibrational presentation of such dualities will combine the benefits of duality-based modal logics and the current results. We are also interested in the relationship to coalgebraic infinite traces (Cîrstea 2011; Jacobs 2004).

Kozen's metric coinduction (Kozen and Ruozzi 2009) is a construction of coinductive predicates by the Banach fixed point theorem and is an alternative to the current paper's order-theoretic one. Its fibrational formulation is an interesting future topic.

Practical applications of our categorical behavioural bounds shall be pursued, too. Our results' precursor - the bounds for the final sequences in Sets (Adámek 2003; Worrell 2005) - have been used to bound execution of some algorithms, e.g. for state minimization (Adámek et al. 2012; Ferrari et al. 2002, 2005). We aim at similar use. Finally, games are an extremely useful tool in fixed point logics (also in their coalgebraic generalization, see Venema (2006), Cîrstea and Sadrzadeh (2008), Cîrstea et al. (2009); 
also, Kupke (2007)). We plan to investigate the use of games in the current (even more general) fibrational setting.

Special Thanks are due to Bart Jacobs and Claudio Hermida for their inspiring work, discussions, comments and encouragements. Besides, thanks are due to Kazuyuki Asada, Keisuke Nakano, Keiko Nakata, Ana Sokolova and the participants of Dagstuhl Seminar 12411 'Coalgebraic Logics' (including Samson Abramsky, Vincenzo Ciancia, Corina Cîrstea, Ernst-Erich Doberkat, Clemens Kupke, Alexander Kurz and Yde Venema) for useful discussions. We are grateful to the anonymous referees, of the earlier version (Hasuo et al. 2013) and the current version, for their careful reading and useful suggestions, too. The authors are supported by Grants-in-Aid No. 23654033, 24680001 \& 15KT0012, JSPS, and by Aihara Innovative Mathematical Modeling Project, FIRST Program, JSPS/CSTP. An earlier version of this paper (Hasuo et al. 2013) has been presented at Mathematical Foundations of Programming Semantics, Twenty-Ninth Annual Conference (MFPS XXIX), 23-25 June 2013, Tulane University, New Orleans, Louisiana, USA. The main part of this work was done when K.C. was an MSc student at Department of Computer Science, the University of Tokyo.

\section{Appendix A. Theory of Coalgebra}

Given a category $\mathbb{C}$ and an endofunctor $F: \mathbb{C} \rightarrow \mathbb{C}$, an $F$-coalgebra is a pair of $X \in \mathbb{C}$ and an arrow $c: X \rightarrow F X$ (we shall denote a coalgebra simply by $X \stackrel{c}{\rightarrow} F X$ ). The notion has turned out to be a useful categorical abstraction of state-based dynamic systems. In an $F$-coalgebra $X \stackrel{c}{\rightarrow} F X$, the carrier object $X \in \mathbb{C}$ is understood as a state space; the functor $F$ specifies the behaviour type; and the arrow $c$ represents actual dynamics. In the most common setting of $\mathbb{C}=$ Sets, examples of functors $F$ (and the corresponding behaviour types) are

- $A \times\left({ }_{-}\right)$for $A$-stream automata;

- $\mathcal{P}(\mathrm{AP}) \times \mathcal{P}\left({ }_{-}\right)$for Kripke models;

$-\mathcal{P}(\mathrm{AP}) \times \mathcal{P}_{\omega}\left({ }_{-}\right)$for finitely branching Kripke models, with where $\mathcal{P}_{\omega}$ is the finite powerset functor;

- $\mathcal{P}\left(A \times \times_{-}\right)$for labelled transition systems;

- $\mathcal{D}\left(A \times{ }_{-}\right)$for generative probabilistic systems;

and so on. See Rutten (2000) and Jacobs (2012) for detailed introduction.

In the theory of coalgebra as a categorical theory of (state-based dynamical) systems, the notion of final coalgebra plays a prominent role. A final $F$-coalgebra $Z \stackrel{\zeta}{\rightarrow} F Z$ is one such that, for any $F$-coalgebra $X \stackrel{c}{\rightarrow} F X$, there is a unique morphism of coalgebras from $c$ to $\zeta$.

$$
\begin{gathered}
F X--\frac{F \bar{c}}{F} \rightarrow F Z \\
c \uparrow \\
X---{ }^{\bar{c}}--\rightarrow Z
\end{gathered}
$$


Its system-theoretic significance is that: $1 Z$ is often the collection of all possible $F$ behaviours'; and 2 the induced arrow $\bar{c}$ assigns, to each state in $X$, its behaviour. The 'behaviours' here follow a black-box view on systems (it ignores internal states) and often captures the natural notion of ' $F$-bisimilarity.'

Therefore, a question arises if a final $F$-coalgebra exists. The well-known Lambek lemma (that $\zeta$ is necessarily an iso) prohibits e.g. a final $\mathcal{P}$-coalgebra. What matters here is the size of $F$ : When it is suitably bounded, a concrete construction of a final coalgebra is known. It obtains a final coalgebra via a final $F$-sequence (Here, 1 is a final object in $\mathbb{C})$.

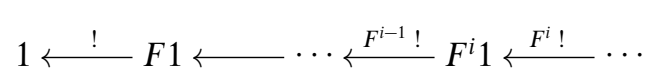

In particular, if $F$ is finitary (a size restriction described later), a final coalgebra arises as a suitable quotient of the limit of the final sequence (4). This construction in Sets is worked out in Worrell (2005); it is further extended to locally presentable categories (those are categories suited for speaking of 'size') with additional assumptions in Adámek (2003). The current paper's goal is to apply this construction also to coinductive predicates.

\section{Appendix B. Locally Finitely Presentable Categories}

The theory of coalgebra has been mainly developed in the base category $\mathbb{C}=$ Sets. Exceptions include the category of nominal sets or (pre)sheaf categories (e.g. Fiore and Staton (2006, 2009)) for name-passing calculi, and Kleisli categories (e.g. Hasuo et al. (2007) and Hasuo (2010)) for trace semantics and simulation. The current paper follows Adámek (2003) and Klin (2007) and finds LFP categories a convenient abstract setting. Here, we follow Adámek and Rosický (1994) and list a minimal set of definitions and results on LFP categories.

The following is a categorical formalization of 'finiteness' of objects. Examples are finite sets (in Sets), and algebras presented by finitely many generators and finitely many equations (in suitable categories of algebras).

Definition B.1 (Finitely presentable object). An object $X \in \mathbb{C}$ is $F P$ if the functor $\mathbb{C}\left(X,{ }_{-}\right): \mathbb{C} \rightarrow$ Sets preserves filtered colimits.

Definition B.2 (Locally finitely presentable category). A category $\mathbb{C}$ is $L F P$ if it is cocomplete and it has a (small) set $\mathbb{F}$ of FP objects such that every object is a filtered colimit of objects in $\mathbb{F}$.

Remark B.3 (Adámek and Rosický (1994, Theorem 1.5)). A filtered colimit can be rewritten as a directed colimit. Hence, every object in an LFP category is a directed colimit of objects in $\mathbb{F}$. Some papers prefer to use directed colimits instead of filtered colimits in the definition of LFP categories, possibly because of simplicity in notations.

Lemma B.4. Let $\mathbb{C}$ be LFP, with a set $\mathbb{F}$ of FP objects as in Definition 3.1; and $X \in \mathbb{C}$. The canonical diagram for $X$ with respect to $\mathbb{F}$

$$
\mathbb{F} / X \stackrel{\pi}{\longrightarrow} \mathbb{F} \longleftrightarrow \mathbb{C}
$$


is filtered, and $X$ is its colimit. Here, $\pi$ is the projection from the comma category $\mathbb{F} / X$ of $\mathbb{F} \hookrightarrow \mathbb{C}$ and $1 \stackrel{X}{\rightarrow} \mathbb{C}$.

Proof. In case $\mathbb{F}$ contains all the FP objects up to isomorphisms, our claim would be Adámek and Rosický (1994, Proposition 1.22). In our current general case, almost the same proof yields our claim, except that we also have to show that the diagram $\mathbb{F} / X$ is filtered.

We shall show that any finite diagram $\left(Y_{I} \stackrel{f_{I}}{\rightarrow} X\right)_{I \in \mathbb{I}}$ in $\mathbb{F} / X$ has its cocone (in $\mathbb{F} / X$ ). First, we construct a cocone in $\mathbb{C} / X$. Let $\left(Y_{I} \stackrel{\kappa_{I}}{\rightarrow} Y\right)_{I}$ be a colimiting cocone in $\mathbb{C}$. The arrows $\left(f_{I}\right)_{I}$ induce $f: Y \rightarrow X$, which forms a colimiting cocone

$$
\left(\left(Y_{I} \stackrel{f_{I}}{\rightarrow} X\right) \stackrel{\kappa_{I}}{\rightarrow}(Y \stackrel{f}{\rightarrow} X)\right)_{I \in \mathbb{I}} \quad \text { in } \mathbb{C} / X
$$

by Lemma B.5 below.

The finite colimit $Y=\operatorname{Colim}_{I} Y_{I}$ of FP objects is FP. Therefore, $Y$ is a split quotient of some object $Y^{\prime}$ in $\mathbb{F}$ (Adámek and Rosický 1994, Remark 1.9). Then we obtain a cocone

$$
\left(\left(Y_{I} \stackrel{f_{I}}{\rightarrow} X\right) \stackrel{i \circ \kappa_{I}}{\longrightarrow}\left(Y^{\prime} \rightarrow Y \stackrel{f}{\rightarrow} X\right)\right)_{I \in \mathbb{I}} \quad \text { in } \mathbb{F} / X
$$

where $i: Y \mapsto Y^{\prime}$ is a section of $Y^{\prime} \rightarrow Y$.

Lemma B.5. Let $\mathbb{C}$ be a cocomplete category and $\left(X_{I}\right)_{I \in \mathbb{I}}$ be a diagram in $\mathbb{C}$. There exists a canonical isomorphism

$$
\left(\left(\underset{I}{\operatorname{Colim}} X_{I}\right) \stackrel{f}{\rightarrow} Y\right) \cong \underset{I}{\operatorname{Colim}}\left(X_{I} \stackrel{f_{I}}{\rightarrow} Y\right) \quad \text { in } \mathbb{C} / Y
$$

for a cocone $\left(X_{I} \stackrel{f_{I}}{\rightarrow} Y\right)_{I}$ and the arrow $f: \operatorname{Colim}_{I} X_{I} \rightarrow Y$ that is induced by the universality of colimits. In other words, the colimiting cocone over $\left(X_{I}\right)_{I}$ in $\mathbb{C}$ induces a colimiting cocone over $\left(f_{I}\right)_{I}$ in $\mathbb{C} / Y$.

Proof. We have a cocone $\left(f_{I} \stackrel{\kappa_{I}}{\rightarrow} f\right)_{I}$ in $\mathbb{C} / Y$ induced by the colimiting cocone $\left(X_{I} \stackrel{\kappa_{I}}{\rightarrow}\right.$ $\left.\operatorname{Colim}_{I} X_{I}\right)_{I}$ in $\mathbb{C}$, since the diagram below commutes and the arrows $f_{I} \stackrel{\kappa_{I}}{\rightarrow} f$ are natural in $I$.

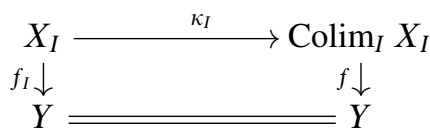

To prove the isomorphism (31), we shall show that the induced cocone, say $c$, is colimiting.

Let $c^{\prime}$ be an arbitrary cocone $\left(f_{I} \stackrel{g_{I}}{\rightarrow} f^{\prime}\right)_{I}$ in $\mathbb{C} / Y$. An arrow $g: \operatorname{Colim}_{I} X_{I} \rightarrow X^{\prime}$ in $\mathbb{C}$ forms an arrow $g: c \rightarrow c^{\prime}$ of cocones if and only if for any $I \in \mathbb{I}$ the diagram

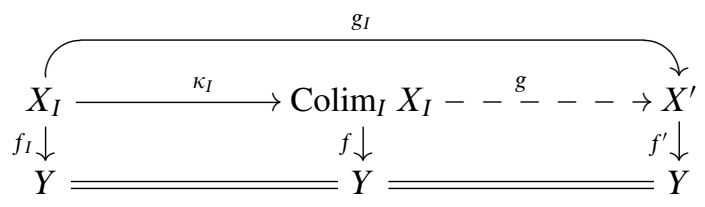

commutes. The universality of colimits in $\mathbb{C}$ shows that an arrow $g$ satisfying $g \circ \kappa_{I}=g_{I}$ for any $I \in \mathbb{I}$ uniquely exists. Moreover, the arrow $g$ with this condition satisfies $f^{\prime} \circ g=f$ 
since $f^{\prime} \circ g \circ \kappa_{I}=f^{\prime} \circ g_{I}=f_{I}$. Hence, there uniquely exists an arrow $g: c \rightarrow c^{\prime}$ of cocones.

Lemma B.6. (Adámek and Rosický 1994, Corollary 1.28 \& Proposition 1.61) Let $\mathbb{C}$ be LFP.

1. $\mathbb{C}$ is complete.

2. $\mathbb{C}$ has (StrongEpi, Mono)- and (Epi, StrongMono)-factorization structures.

For each $X \in \mathbb{C}$, the (StrongEpi, Mono)-factorization structure induces the image functor $\operatorname{Im}: \mathbb{C} / X \rightarrow \operatorname{Sub}(X)$, which is left adjoint to the forgetful functor $\operatorname{Sub}(X) \rightarrow \mathbb{C} / X$. An image of a colimit can be calculated as a sup of images.

Lemma B.7. Let $\mathbb{C}$ be LFP and $\left(X_{I} \stackrel{\kappa_{I}}{\rightarrow} X\right)$ be a colimiting cocone in $\mathbb{C}$. For an arbitrary cocone $\left(X_{I} \stackrel{f_{I}}{\rightarrow} Y\right)$, we have

$$
\operatorname{Im} f=\bigvee_{I} \operatorname{Im} f_{I} \quad \text { in } \operatorname{Sub}(Y)
$$

where $f: X \rightarrow Y$ is induced by the universality of colimits.

Proof. We have

$$
\operatorname{Im}\left(\left(\underset{I \in \mathbb{I}}{\operatorname{Colim}} X_{I}\right) \stackrel{f}{\rightarrow} Y\right)=\operatorname{Im}\left(\operatorname{Colim}_{I \in \mathbb{I}}\left(X_{I} \stackrel{f_{I}}{\rightarrow} Y\right)\right)=\bigvee_{I \in \mathbb{I}} \operatorname{Im}\left(X_{I} \stackrel{f_{I}}{\rightarrow} Y\right)
$$

The former equality is by Lemma B.5; the latter is because $\operatorname{Im}: \mathbb{C} / Y \rightarrow \operatorname{Sub}(Y)$ is a left adjoint functor.

Lemma B.8. Let $\mathbb{C}$ be an LFP category.

1. Adámek and Rosický (1994, Proposition 1.59) filtered colimits commute with finite limits in $\mathbb{C}$. Precisely, the canonical arrow

$$
\underset{I \in \mathbb{I}}{\operatorname{Colim}} \operatorname{Lim}_{J \in \mathbb{J}} X_{I, J} \rightarrow \underset{J \in \mathbb{J}}{\operatorname{Lim}} \underset{I \in \mathbb{I}}{\operatorname{Colim}} X_{I, J}
$$

is an isomorphism for a diagram $\left(X_{I, J}\right)_{(I, J) \in \mathbb{I} \times \mathbb{J}}$ in $\mathbb{C}$ such that $\mathbb{I}$ is a filtered category and $\mathbb{J}$ is a finite category.

2. Filtered colimits in $\mathbb{C}$ are stable under pullbacks.

Proof. We prove the item 2. Let $X=\operatorname{Colim}_{I \in \mathbb{I}} X_{I}$ be a filtered colimit and $f: Y \rightarrow X$ be an arrow. Apply the item 1 to the diagram

$$
\left(\begin{array}{c}
Y \\
X_{I} \underset{\kappa_{I}}{\downarrow} X^{\downarrow} f
\end{array}\right)_{I \in \mathbb{I}}
$$


where $\mathbb{J}=\left(\begin{array}{l} \\ \downarrow \\ \rightarrow\end{array}\right)$. This yields a pullback square
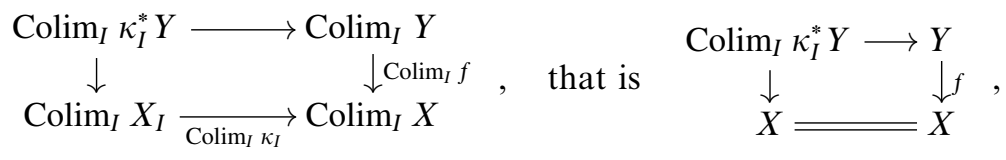

because we have $X=\operatorname{Colim}_{I \in \mathbb{I}} X$ and $Y=\operatorname{Colim}_{I \in \mathbb{I}} Y$ for a filtered category $\mathbb{I}$. Since a pullback of $f: Y \rightarrow X$ along id $: X \rightarrow X$ is given by $f$ itself, we obtain $\operatorname{Colim}_{I} \kappa_{I}^{*} Y=Y$, as required.

The following notion (which is already in Definition B.1) is about the 'size' of functors. An intuition (when $\mathbb{C}=$ Sets) is: a functor $F$ is finitary if $F$ 's action $F X$ on an arbitrary set $X$ is determined by its action $F X^{\prime}$ on all the finite subsets $X^{\prime} \subseteq X$.

Definition B.9 (Finitary functor). A functor $F: \mathbb{C} \rightarrow \mathbb{D}$ is finitary if it preserves filtered colimits.

For an endofunctor $F: \mathbb{C} \rightarrow \mathbb{C}$, this notion of finitariness is commonly used to bound the 'branching degree' of systems as $F$-coalgebras. For example, the finite powerset functor $\mathcal{P}_{\omega}$ is finitary; the (full) powerset functor $\mathcal{P}$ is not.

There are many LFP categories, among which are Sets, the category Posets of posets and monotone functions, and categories of algebras with finitary operations. See Adámek and Rosický (1994) for more examples.

Example B.10 (Presheaf categories). Let $\mathbb{A}$ be a small category. The presheaf category Sets $^{\mathbb{A}}$ is LFP: the set

$$
\mathbb{F}:=\{\text { finite colimits of representable presheaves } \mathbf{y} A\},
$$

where $\mathbf{y} A=\mathbb{A}\left(A,,_{-}\right)$, satisfies the conditions of Definition B.1.

Lemma B.11. Let $\mathbb{C}$ be LFP, with $\mathbb{F} \subseteq \mathbb{C}$ as in Definition 3.1; and $X \in \mathbb{C}$.

1. Adámek and Rosický (1994, Proposition 1.57) The slice category $\mathbb{C} / X$ is LFP, which is guaranteed by the set $\mathbb{F}_{\mathbb{C} / X}=\mathbb{F} / X$ of FP objects.

2. The poset $\operatorname{Sub}(X)$ of subobjects is LFP (i.e. it is an algebraic lattice, meaning a complete lattice in which each element is a join of compact elements), which is guaranteed by the set

$$
\begin{aligned}
\mathbb{F}_{\operatorname{Sub}(X)} & =\{\operatorname{Im} f \mid f \in \mathbb{F} / X\} \\
& =\{(P \mapsto X) \mid \text { there exist an object } Z \in \mathbb{F} \text { and a strong epi } Z \rightarrow P\},
\end{aligned}
$$

of FP objects (i.e. compact elements) where $\operatorname{Im}: \mathbb{C} / X \rightarrow \operatorname{Sub}(X)$ denotes the image functor defined by the (StrongEpi, Mono)-factorization.

Proof. We shall prove the item 2. A proof that $\operatorname{Sub}(X)$ is LFP without explicit description of $\mathbb{F}_{\operatorname{Sub}(X)}$ is found e.g. in Porst (2011, Theorem 5). 
The lattice $\operatorname{Sub}(X)$ is a reflective subcategory of $\mathbb{C} / X$ by the reflection $\operatorname{Im}: \mathbb{C} / X \rightarrow$ $\operatorname{Sub}(X)$. Thus, $\operatorname{Sub}(X) \subseteq \mathbb{C} / X$ is closed under filtered colimits by Adámek and Rosický (1994, Corollary 1.60). Hence, by Adámek and Rosický (1994, Theorem 1.39), Sub $(X)$ is LFP, with FP objects $\{\operatorname{Im} f \mid f \in \mathbb{F} / X\}$.

\section{Appendix C. Fibrations}

We follow Jacobs (1999), although we focus on the simpler notion of poset fibration.

\section{C.1. Introduction (via Indexed Posets)}

This paper's interest is in coinductive predicates, hence in predicate logic. The most straightforward formalization of predicate is as a subset $P \subseteq X$ of a set (a 'universe') $X$ : an element $x \in X$ satisfies $P$ if $x \in P$. Accompanying is the natural notion of entailment: $P$ entails $Q$ if $P \subseteq Q$. This way we obtain the poset $\left(2^{X}, \subseteq\right)$ of predicates over $X$.

However, it is not on a single universe $X$ that we consider predicates. For example, in a situation where there are two Kripke models $c=\left(X, \rightarrow, V_{X}\right), d=\left(Y, \rightarrow, V_{Y}\right)$ and a 'homomorphism' $f: X \rightarrow Y$, a natural question is if the interpretation of a formula $v u . \alpha$ is preserved by $f$. (It is; see Proposition 3.13). Here, we are comparing the predicate $\llbracket v u . \alpha \rrbracket_{c} \subseteq X$ with the predicate $\llbracket v u . \alpha \rrbracket_{d} \subseteq Y$ reindexed via $f: X \rightarrow Y$. The latter is concretely described as the inverse image

$$
f^{-1}\left(\llbracket v u . \alpha \rrbracket_{d}\right)=\left\{x \in X \mid f(x) \in \llbracket v u . \alpha \rrbracket_{d}\right\} .
$$

Therefore, a reindexing structure is also relevant to predicate logic: a function $f: X \rightarrow Y$ induces reindexing $f^{-1}: 2^{Y} \rightarrow 2^{X}$. Additionally, the map $f^{-1}$ is monotone.

To summarize: (1) predicates on a universe $X$ form a poset; (2) a function $f: X \rightarrow$ $Y$ between universes induces a monotone reindexing function from the collection of predicates over $X$ to that over $Y$. Such a situation is nicely described as a (contravariant) functor

$$
\Phi: \mathbb{C}^{\mathrm{op}} \longrightarrow \text { Posets }
$$

where Posets is the category of posets and monotone functions. The functor $\Phi$ assigns, to each 'universe' $X \in \mathbb{C}$, the poset $\Phi X$ of predicates over $X$. Moreover, $f: X \rightarrow Y$ in $\mathbb{C}$ induces a reindexing map $\Phi f: \Phi Y \rightarrow \Phi X$. This functor $\Phi$ is a special case of an indexed category (Jacobs 1999, Section 1.10).

In the current paper, however, we favour an equivalent presentation of such a structure by a fibration, since we find the latter to be more amenable to generalization of structures in ordinary category theory (such as limits). The equivalence between index categories and fibrations is well-known; here, we sketch the Grothendieck construction from the former to the latter. Its idea is to 'patch up' the posets $(\Phi X)_{X \in \mathbb{C}}$ and form a big category $\mathbb{P}$, as 
in the following figure:

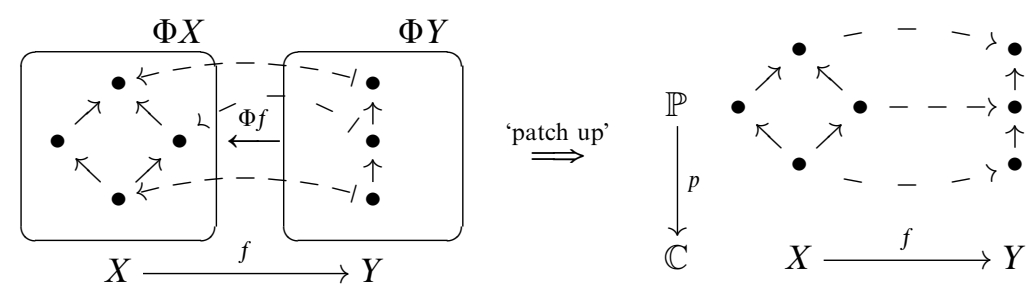

On the right, we add some arrows (denoted by $-\rightarrow)$ so that we have an arrow $(\Phi f)(Q) \rightarrow Q$ in $\mathbb{P}$ for each $Q \in \Phi Y$. (On the left the correspondence, $1-\rightarrow$ depicts the action of the map $\Phi f$.) The above diagram in $\mathbb{P}$ should be understood as a Hasse diagram: those arrows which arise from composition are not depicted.

Formally:

Definition C.1 (The Grothendieck construction). Given $\Phi: \mathbb{C}^{\text {op }} \rightarrow$ Posets, we define the category $\mathbb{P}_{\Phi}$ by

- its object is a pair $(X, P)$ of an object $X \in \mathbb{C}$ and an element $P$ of the poset $\Phi X$; and - its arrow $f:(X, P) \rightarrow(Y, Q)$ is an arrow $f: X \rightarrow Y$ in $\mathbb{C}$ such that

$$
P \leqslant(\Phi f)(Q)
$$

Here, $\leqslant$ refers to the order of $\Phi X$.

Thus arises a category $\mathbb{P}=\mathbb{P}_{\Phi}$ that incorporates: the order structure of each of the posets $(\Phi X)_{X \in \mathbb{C}}$; and the reindexing structure by $(\Phi f)_{f}: \mathbb{C}$-arrow. For fixed $X \in \mathbb{C}$, the objects of the form $(X, P)$ and the arrows $\mathrm{id}_{X}$ between them form a subcategory of $\mathbb{P}$. This is denoted by $\mathbb{P}_{X}$ and called the fibre over $X$. It is obvious that $\mathbb{P}_{X}$ is a poset that is isomorphic to $\Phi X$.

Moreover, there is a canonical projection functor $p: \mathbb{P} \rightarrow \mathbb{C}$ that carries $(X, P)$ to $X$.

\section{C.2. Formal definition of (Poset) fibration}

We axiomatize those structures which arise in the way described above.

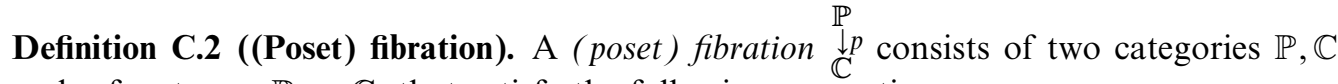
and a functor $p: \mathbb{P} \rightarrow \mathbb{C}$, that satisfy the following properties:

- Each fibre $\mathbb{P}_{X}$ is a poset. Here, the fibre $\mathbb{P}_{X}$ for $X \in \mathbb{C}$ is the subcategory of $\mathbb{P}$ consisting of objects $P \in \mathbb{P}$ such that $p P=X$ and arrows $f: P \rightarrow Q$ such that $p f=\mathrm{id}_{X}$ (such arrows are said to be vertical).

- Given $f: X \rightarrow Y$ in $\mathbb{C}$ and $Q \in \mathbb{P}_{Y}$, there is an object $f^{*} Q \in \mathbb{P}_{X}$ and a $\mathbb{P}$-arrow $\bar{f} Q: f^{*} Q \rightarrow Q$ with the following universal property. For any $P \in \mathbb{P}_{X}$ and $g: P \rightarrow Q$ in $\mathbb{P}$, if $p g=f$, then $g$ factors through $\bar{f}(Q)$ uniquely via a vertical arrow. That is, 
there exists a unique $g^{\prime}$ such that $g=\bar{f}(Q) \circ g^{\prime}$ and $p g^{\prime}=\mathrm{id}_{X}$.

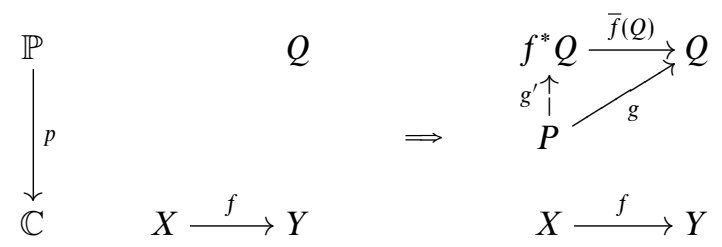

— The correspondences $\left({ }_{-}\right)^{*}$ and $\overline{\left(\_\right.}$are functorial:

$$
\begin{aligned}
\operatorname{id}_{Y}^{*} Q & =Q, & (g \circ f)^{*}(Q) & =f^{*}\left(g^{*} Q\right), \\
\overline{\operatorname{id}_{Y}}(Q) & =\operatorname{id}_{Q}, & \overline{g \circ f}(Q) & =\bar{g} Q \circ \bar{f}\left(g^{*} Q\right) .
\end{aligned}
$$

The last equality can be depicted as follows:

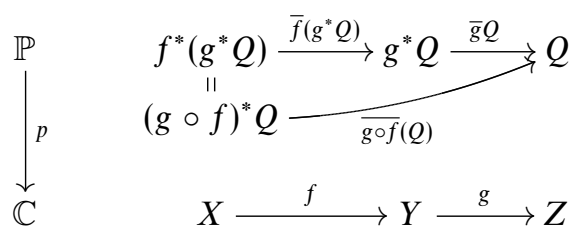

The category $\mathbb{P}$ is called the total category of the fibration; $\mathbb{C}$ is the base category. The arrow $\bar{f} Q: f^{*} Q \rightarrow Q$ is called the Cartesian lifting of $f$ and $Q$. An arrow in $\mathbb{P}$ is Cartesian (or reindexing) if it coincides with $\bar{f} Q$ for some $f$ and $Q$.

In the case where $\underset{\mathbb{C}}{\mathbb{P}}$ is induced by an indexed category $\Phi: \mathbb{C}^{\text {op }} \rightarrow$ Posets via Definition C.1, a Cartesian lifting is obviously given by $f^{*}(Q)=(\Phi f)(Q)$.

In the current paper, we focus on poset fibrations (which we shall simply call fibrations). In a (general) fibration, a fibre $\mathbb{P}_{X}$ is not just a poset but a category, and this elicits a lot of technical subtleties. Nevertheless, it should not be hard to generalize the current paper's results to general, not necessarily poset, fibrations (especially to the split ones). We shall often denote a vertical arrow in $\mathbb{P}$ (i.e. an arrow inside a fibre) by $\leqslant$.

The dual notion of a fibration is an opfibration.

Definition C.3. An opfibration $\underset{\mathbb{C}}{\mathbb{P}}$ consists of two categories $\mathbb{P}, \mathbb{C}$ and a functor $p: \mathbb{P} \rightarrow \mathbb{C}$ such that $\underset{\mathbb{C}^{\text {op }}}{\stackrel{\downarrow}{\text { op }}}$ is a fibration. Concretely, in an opfibration $\underset{\mathbb{C}}{\stackrel{\mathbb{P}}{\downarrow}}$, for an arrow $f: X \rightarrow Y$ in $\mathbb{C}$ and $P \in \mathbb{P}_{X}$, there is an object $\coprod_{f} P \in \mathbb{P}_{Y}$ and a $\mathbb{P}$-arrow $P \rightarrow \coprod_{f} P$ satisfying an appropriate universal property. This arrow $P \rightarrow \coprod_{f} P$ in $\mathbb{P}$ is said to be opcartesian (or opreindexing).

A bifibration $\underset{\mathbb{C}}{\stackrel{\mathbb{P}}{\downarrow p}}$ is a fibration as well as an opfibration.

Note that we do not assume the Beck-Chevalley condition for a bifibration. A fibration with coproducts $\coprod_{f}$ between fibres - introduced later in Definition C.10-carries a canonical opfibration structure, too. 


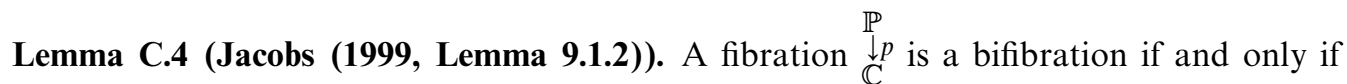
for any arrow $f: X \rightarrow Y$ in $\mathbb{C}$ the reindexing functor $f^{*}: \mathbb{P}_{Y} \rightarrow \mathbb{P}_{X}$ has a left adjoint $\coprod_{f} \dashv f^{*}$.

\section{C.3. Examples}

Example C.5 (Subobject fibration). Let $\mathbb{C}$ be a (well-powered) category with finite limits. The category $\operatorname{Sub}(\mathbb{C})$ is defined by: its object is a pair $(P, X)$ of $X \in \mathbb{C}$ and its subobject $P \longmapsto X$ (we write $(P \mapsto X) \in \operatorname{Sub}(\mathbb{C}))$; and its arrow $(P \mapsto X) \stackrel{f}{\rightarrow}(V \mapsto Y)$ is a $\mathbb{C}$-arrow $f: X \rightarrow Y$ that restricts to $P \rightarrow Q$. That is, given an arrow $f: X \rightarrow Y$ in $\mathbb{C}$,

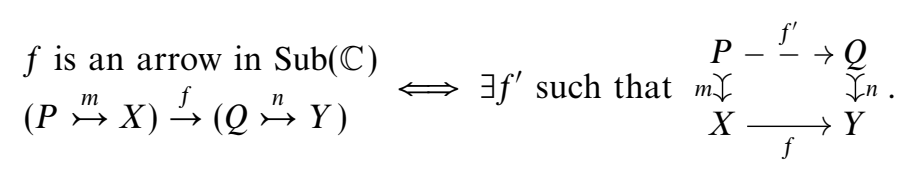

The projection $(P \longmapsto X) \mapsto X$ defines a functor; thus arises the subobject fibration $\underset{\mathfrak{C}}{\stackrel{\downarrow}{\mathbb{C}}}$ of $\mathbb{C}$. In particular, given $X \stackrel{f}{\rightarrow} Y$ in $\mathbb{C}$ and $(Q \mapsto Y) \in$ $\operatorname{Sub}(Y)$, the Cartesian lifting $f^{*} Q$ is defined by a pullback.

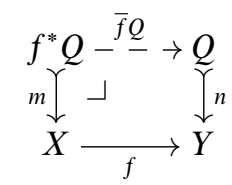

A special case is the following most straightforward modelling of predicate logic. It arises from the contravariant powerset functor $2_{-}^{(-)}:$Sets $^{\text {op }} \rightarrow$ Posets via Definition C.1.

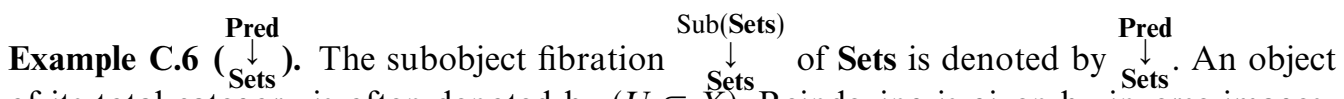
of its total category is often denoted by $(U \subseteq X)$. Reindexing is given by inverse images.

More concretely, in the category Pred, an object is a pair $(P, X)$ of a set $X$ and its subset $P \subseteq X$; an arrow $(P \subseteq X) \stackrel{f}{\rightarrow}(Q \subseteq Y)$ is a function $X \stackrel{f}{\rightarrow} Y$ that restricts to $P \rightarrow Q$ (i.e. $P \subseteq f^{-1} Q$ ).

Example C.7 (Rel). The fibration $\underset{\text { Sets }}{\substack{\downarrow \\ \text { cel }}}$ can be introduced from $\underset{\text { Sets }}{\downarrow}$ via the following change-of-base:

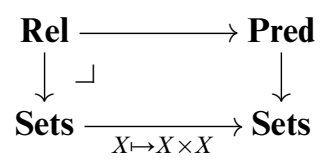

Concretely, an object of Rel is a pair $(X, R)$ of a set $X$ and a relation $R \subseteq X \times X$; an arrow $f:(X, R) \rightarrow(Y, S)$ is a function $f: X \rightarrow Y$ such that $x R x^{\prime}$ implies $f(x) S f\left(x^{\prime}\right)$. See Jacobs (1999, p. 14).

Example C.8 (Family fibration). The family fibration $\underset{\text { Sets }}{\downarrow}$ over a poset $\Omega$ is introduced as follows. An object in the fibre $\operatorname{Fam}(\Omega)_{X}$ is a function $f: X \rightarrow \Omega$; and an arrow $(X \stackrel{f}{\rightarrow} \Omega) \stackrel{k}{\rightarrow}(Y \stackrel{g}{\rightarrow} \Omega)$ in the total category $\operatorname{Fam}(\Omega)$ is a function $k: X \rightarrow Y$ such that $f(x) \leqslant g(k(x))$ for each $x \in X$. See e.g. Jacobs (1999, Definition 1.2.1) for more details. 


\section{C.4. Structures in a fibration}

In a fibration $\underset{\mathbb{C}}{\mathbb{P}}$, a $\mathbb{C}$-arrow $X \stackrel{f}{\rightarrow} Y$ induces a correspondence $\mathbb{P}_{Y} \stackrel{f^{*}}{\rightarrow} \mathbb{P}_{X}$ via reindexing. This is easily seen to be a monotone map (i.e. a functor between posets as categories).

Definition C.9 (Fibrewise (co)limits). A fibration $\underset{\mathbb{C}}{\mathbb{P}}$ is said to have fibrewise limits if - each fibre $\mathbb{P}_{X}$ has, as a category, all limits (meaning it has arbitrary inf's $\bigwedge$ ); and

- for each $\mathbb{C}$-arrow $X \stackrel{f}{\rightarrow} Y$, the reindexing functor $\mathbb{P}_{Y} \stackrel{f^{*}}{\rightarrow} \mathbb{P}_{X}$ preserves these limits.

In this case, each fibre $\mathbb{P}_{X}$ has a final object (denoted by $\top_{X}$ ).

Similarly, a fibration has fibrewise colimits if each fibre has them and they are preserved by reindexing.

The following notions must be distinguished from 'fibrewise (co)products.'

Definition C.10 ((Co)products between fibres). A fibration $\underset{\mathbb{C}}{\mathbb{P}}$ is said to have products (between fibres) if

— each reindexing functor $f^{*}: \mathbb{P}_{Y} \rightarrow \mathbb{P}_{X}$ has a right adjoint $f^{*} \dashv \prod_{f}$; and

- the functors $\left(\prod_{f}\right)_{f}$ satisfy the so-called Beck-Chevalley condition. See Jacobs (1999, Section1.9).

Similarly, a fibration has coproducts (between fibres) if each reindexing has a left adjoint $\coprod_{f}$ and they satisfy the Beck-Chevalley condition.

The prototype example $\underset{\downarrow}{\downarrow} \stackrel{\text { Pred }}{\downarrow}$ has fibrewise (co)limits: each fibre is a complete lattice; and $\bigwedge$ and $\bigvee$ are preserved by inverse images. It has products $\Pi$ and coproducts $\amalg$ between fibres, too: specifically $\coprod_{f}$ is given by the direct image of the function $f$. See Jacobs (1999, Section 1.9).

Throughout the paper, we rely on the following result. It extends Lemma 3.6. Note that colimits are preserved by opreindexings in a bifibration.

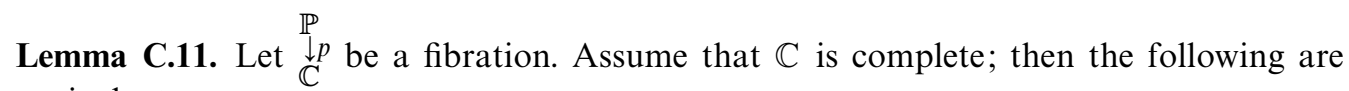
equivalent:

1. The fibration $p$ has fibrewise limits.

2. The total category $\mathbb{P}$ is complete and $p: \mathbb{P} \rightarrow \mathbb{C}$ preserves limits.

If this is the case, a limit of a small diagram $\left(P_{I}\right)_{I \in \mathbb{I}}$ in $\mathbb{P}$ can be given by

$$
\bigwedge_{I \in \mathbb{I}}\left(\pi_{I}^{*} P_{I}\right) \quad \text { over } \operatorname{Lim}_{I \in \mathbb{I}} X_{I} \text {. }
$$

Here, $X_{I}:=p P_{I} ;\left(\operatorname{Lim}_{I \in \mathbb{I}} X_{I} \stackrel{\pi_{I}}{\rightarrow} X_{I}\right)_{I \in \mathbb{I}}$ is a limiting cone in $\mathbb{C}$; and $\bigwedge_{I \in \mathbb{I}}$ denotes the limit computed in the fibre $\mathbb{P}_{\mathbb{P}} X_{I} X_{I}$.

(Sort of) dually, let $\underset{\mathbb{C}}{\downarrow}$ be a bifibration (such as a fibration with coproducts $\amalg$ between fibres, see Lemma C.4). Assume that $\mathbb{C}$ is cocomplete; then the following are equivalent:

1. Any fibre $\mathbb{P}_{X}$ has colimits.

2. The total category $\mathbb{P}$ is cocomplete and $p: \mathbb{P} \rightarrow \mathbb{C}$ preserves colimits. 
In this case, a colimit of a small diagram $\left(P_{I}\right)_{I \in \mathbb{I}}$ in $\mathbb{P}$ can be given by

$$
\bigvee_{I \in \mathbb{I}}\left(\coprod_{\kappa_{I}} P_{I}\right) \quad \text { over } \operatorname{Colim}_{I} X_{I}
$$

where $X_{I}:=p P_{I}$ and $\left(X_{I} \stackrel{\kappa_{I}}{\rightarrow} \operatorname{Colim}_{I} X_{I}\right)_{I \in \mathbb{I}}$ is a colimiting cocone in $\mathbb{C}$.

In contrast to the above results that are on limits in the total category $\mathbb{P}$ of a fibration, Lemma C.12 allows one to compute limits over $\mathbb{P}$ as a diagram. It is well known that an

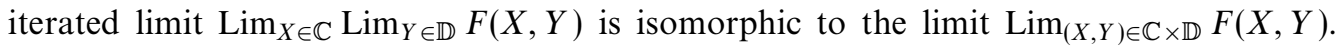
This kind of isomorphism exists even if the category $\mathbb{D}$ 'depends' on $X \in \mathbb{C}$ in the

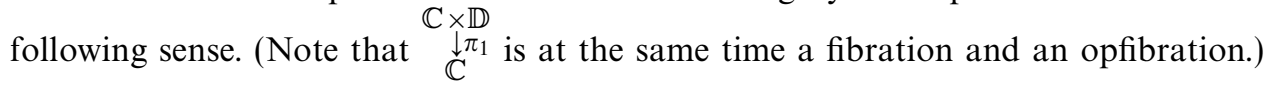

Lemma C.12. Let $\underset{\mathbb{C}}{\stackrel{\mathbb{P}}{\downarrow}}$ be a fibration and $F: \mathbb{P} \rightarrow \mathbb{E}$ be a functor. If $\operatorname{Lim}_{P \in \mathbb{P}_{X}} F P$ exists for each $X \in \mathbb{C}$, then we have a canonical isomorphism

$$
\operatorname{Lim}_{X \in \mathbb{C}} \operatorname{Lim}_{P \in \mathbb{P}_{X}} F P \cong \operatorname{Lim}_{P \in \mathbb{P}} F P
$$

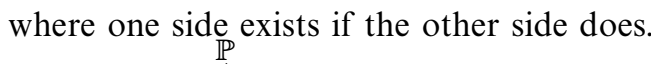

Dually, let $\underset{\mathbb{C}}{\downarrow p}$ be an opfibration and $F: \mathbb{P} \rightarrow \mathbb{E}$ be a functor. If $\operatorname{Colim}_{P \in \mathbb{P}_{X}} F P$ exists for each $X \in \mathbb{C}$, then we have a canonical isomorphism

$$
\underset{X \in \mathbb{C}}{\operatorname{Colim}} \underset{P \in \mathbb{P}_{X}}{\operatorname{Colim}} F P \cong \underset{P \in \mathbb{P}}{\operatorname{Colim}} F P,
$$

where one side exists if the other side does.

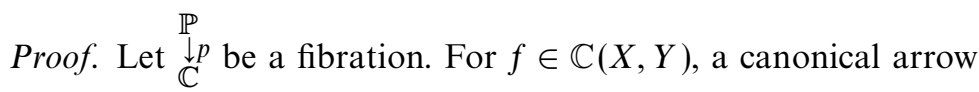

$$
\operatorname{Lim}_{P \in \mathbb{P}_{X}} F P \rightarrow \operatorname{Lim}_{Q \in \mathbb{P}_{Y}} F Q
$$

is obtained via the universality of limits as below:

$$
\begin{gathered}
\operatorname{Lim}_{P \in \mathbb{P}_{X}} F P--\rightarrow \operatorname{Lim}_{Q \in \mathbb{P}_{Y}} F Q \\
\pi_{f^{*} Q \downarrow} \downarrow^{\pi_{Q}} \\
F\left(f^{*} Q\right) \underset{F(\bar{f} Q)}{\longrightarrow} F Q .
\end{gathered}
$$

Indeed, we have

$$
\begin{array}{cc}
F\left(f^{*} Q\right) \stackrel{F(\bar{f} Q)}{\longrightarrow} F Q \\
F\left(f^{*} g\right) \downarrow \\
F\left(f^{*} Q^{\prime}\right) \underset{ }{\downarrow} \underset{F\left(\bar{f} Q^{\prime}\right)}{\longrightarrow} F Q^{\prime}
\end{array}
$$

for $g \in \mathbb{P}_{Y}\left(Q, Q^{\prime}\right)$ because of the naturality of Cartesian liftings. 
For each $E \in \mathbb{E}$, we have

$$
\begin{aligned}
& \mathbb{E}\left(E, \operatorname{Lim}_{X \in \mathbb{C}} \operatorname{Lim}_{P \in \mathbb{P}_{X}} F P\right) \\
& \cong \operatorname{Lim}_{X \in \mathbb{C}} \operatorname{Lim}_{P \in \mathbb{P}_{X}} \mathbb{E}(E, F P) \\
& \cong \operatorname{Lim}_{X \in \mathbb{C}}\left\{\left(h_{P} \in \mathbb{E}(E, F P)\right)_{P \in \mathbb{P}_{X}} \mid F g \circ h_{P}=h_{P^{\prime}} \text { for any } g \in \mathbb{P}_{X}\left(P, P^{\prime}\right)\right\} \\
& \cong\left\{\left(\left(h_{P} \in \mathbb{E}(E, F P)\right)_{P \in \mathbb{P}_{X}}\right)_{X \in \mathbb{C}}\right. \\
& \mid F g \circ h_{P}=h_{P^{\prime}} \text { for any } g \in \mathbb{P}_{X}\left(P, P^{\prime}\right) ; \\
& \left.F(\bar{f} Q) \circ h_{f^{*} Q}=h_{Q} \text { for any } f \in \mathbb{C}(X, Y) \text { and } Q \in \mathbb{P}_{Y}\right\}
\end{aligned}
$$

the postcomposition of the arrow (35) maps $\left(h_{P}\right)_{P}$ to $\left(F(\bar{f} Q) \circ h_{f^{*} Q}\right)_{Q}$ by Equation (36) $=\left\{\left(h_{P} \in \mathbb{E}(E, F P)\right)_{P \in \mathbb{P}} \mid F f \circ h_{P}=h_{Q}\right.$ for any $\left.f \in \mathbb{P}(P, Q)\right\}$

by the factorization $P \stackrel{g}{\rightarrow} f^{*} Q \stackrel{\bar{f}}{\rightarrow} Q$ of $f: P \rightarrow Q$ into vertical $g$ and Cartesian $\bar{f}$

$$
\begin{aligned}
& \cong \operatorname{Lim}_{P \in \mathbb{P}} \mathbb{E}(E, F P) \\
& =\mathbb{E}\left(E, \operatorname{Lim}_{P \in \mathbb{P}} F P\right) .
\end{aligned}
$$

Applying the Yoneda Lemma yields the claim.

\section{C.5. Fibrewise opposite}

Let op : Posets $\rightarrow$ Posets be a functor that maps $(\mathbf{P}, \leqslant)$ to $(\mathbf{P}, \leqslant)^{\mathrm{op}}=(\mathbf{P}, \geqslant)$. Assuming a fibration $\underset{\mathbb{C}}{\mathbb{P}}$ is induced - by the Grothendieck construction - by an indexed category $\Phi: \mathbb{C}^{\text {op }} \rightarrow$ Posets, the composite $\mathbb{C}^{\text {op }} \stackrel{\Phi}{\rightarrow}$ Posets $\underset{\mathbb{P}^{(\mathrm{op})}}{\stackrel{\text { op }}{\rightarrow}}$ Posets induces a fibration in which each fibre is opposed. This is what is denoted by $\underset{\mathbb{C}}{\downarrow p^{(\mathrm{op})}}$ in the following lemma.

Lemma C.13 (Fibrewise opposite, Bénabou (1975)). Let $\underset{\mathbb{C}}{\stackrel{\mathbb{P}}{\downarrow p}}$ be a fibration. There exists a canonical fibration $\underset{\mathbb{C}}{\stackrel{\mathbb{P}}{\mathbb{P}^{(\mathrm{op})}} \text { (op) }}$ such that $\left(\mathbb{P}^{(\mathrm{op})}\right)_{X}=\left(\mathbb{P}_{X}\right)^{\mathrm{op}}$, and reindexing functors coincide, as in the commutative diagram

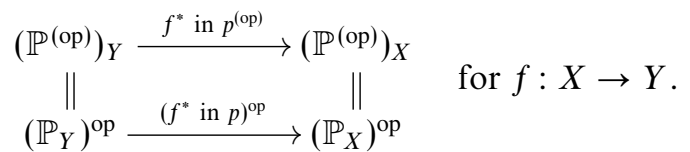

This fibration $p^{(\mathrm{op})}$ is called the fibrewise opposite of $p$.

Proof. We describe the construction of $\mathbb{P}^{(\mathrm{op})}$; it is simple in the current setting where we focus on poset fibrations. The objects are the same as those of $\mathbb{P}$, and the arrows are defined by

$$
\mathbb{P}^{(\mathrm{op})}(P, Q)=\left\{f: p P \rightarrow p Q \mid P \geqslant f^{*} Q\right\}
$$


It is easy to show that $\operatorname{id}_{p P}$ induces an arrow $P \rightarrow P$ in $\mathbb{P}^{(\mathrm{op})}$; this gives the identity arrow. The composite $g \circ f \in \mathbb{P}^{(\mathrm{op})}(P, R)$ of $f \in \mathbb{P}^{(\mathrm{op})}(P, Q)$ and $g \in \mathbb{P}^{(\mathrm{op})}(Q, R)$ is given by composition in $\mathbb{C}$, too.

\section{Appendix D. Omitted proofs}

\section{D.1. Proof of Proposition 6.16}

We shall prove the item 1. In the topos $\operatorname{Sets}^{\mathbb{A}}$, there exists an (Epi,Mono)-factorization, which induces the image functor $\operatorname{Im}: \operatorname{Sets}^{\mathbb{A}} / P \rightarrow \operatorname{Sub}(P)$ that is surjective on objects. In particular, a subpresheaf of $P$ can be thought of as an image of some arrow with codomain $P$.

Let $(Q \stackrel{\theta}{\longrightarrow} P) \in \operatorname{Sets}^{\mathbb{A}} / P$. By Lemma 6.14, we may assume $Q=\operatorname{Colim}_{I \in \mathbb{I}}\left(\mathbf{y} B_{I}\right)$ for some diagram $\left(B_{I}\right)_{I \in \mathbb{I}}$. By Example 6.10 and Lemma B.7, we have $\operatorname{Im} \theta=\bigvee_{I \in \mathbb{I}} \operatorname{Im} \theta_{I}$ where the arrow $\theta_{I}$ is the composite $\left(\mathbf{y} B_{I} \rightarrow \operatorname{Colim}_{I}\left(\mathbf{y} B_{I}\right) \stackrel{\theta}{\rightarrow} P\right)$.

Letting $P=\mathbf{y} A$, we obtain $\operatorname{Im}(Q \stackrel{\theta}{\longrightarrow} \mathbf{y} A)=\bigvee_{I} \operatorname{Im}\left(\mathbf{y} B_{I} \stackrel{\mathbf{y} f_{I}}{\longrightarrow} \mathbf{y} A\right)$ for a family $\left(f_{I}\right)_{I}$ such that $\theta_{I}=\mathbf{y} f_{I}$; such a family $\left(f_{I}\right)_{I}$ exists since the functor $\mathbf{y}$ is full and faithful. This proves the item 1 .

We shall now prove the item 2 . We observe that an epi $A \rightarrow C$ in $\mathbb{A}$ induces a mono $\mathbf{y} C \mapsto \mathbf{y} A$ in Sets $^{\mathbb{A}}:$ this is because the functor $\mathbf{y}: \mathbb{A}^{\text {op }} \rightarrow$ Sets $^{\mathbb{A}}$ preserves all existing limits, including the pullback

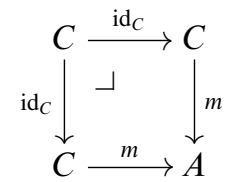

in $\mathbb{A}^{\text {op }}$. (The diagram is a pullback if and only if $m$ is a mono in $\mathbb{A}^{\text {op }}$, i.e. an epi in $\mathbb{A}$.) Thus, there is a monotone function $\operatorname{Quot}(A) \rightarrow \operatorname{Sub}(\mathbf{y} A)$.

Regarding monos in $\mathbb{A}$, we can show the following sublemma (its only-if direction will not be used later).

Sublemma D.1. Let $m: C \rightarrow B$ be an arrow in $\mathbb{A}$. The arrow $\mathbf{y} m: \mathbf{y} B \rightarrow \mathbf{y} C$ is an epi in Sets $^{\mathbb{A}}$ if and only if the arrow $m$ is a split mono.

Proof. The following are equivalent (folklore): For an arrow $e$ in $\mathbb{B}$,

1. the arrow $e$ is an absolute epi, i.e. $F(e)$ is an epi for any functor $F$ with the domain $\mathbb{B}$,

2. the arrow ye in Sets ${ }^{\mathbb{B}^{\text {op }}}$ is an epi, and

3. the arrow $e$ is a split epi.

The sublemma is part of this fact for $\mathbb{B}=\mathbb{A}^{\mathrm{op}}$.

To be concrete, we take a retraction $r: B \rightarrow C$ of a split mono $m$ in $\mathbb{A}$. By $r \circ m=\mathrm{id}_{C}$, we have $\mathbf{y} m \circ \mathbf{y} r=\mathrm{id}_{\mathbf{y} C}$, which shows that $\mathbf{y} m$ is a (split) epi in Sets ${ }^{\mathbb{A}}$.

Conversely, let $m: C \rightarrow B$ be an arrow in $\mathbb{A}$ such that $\mathbf{y} m: \mathbf{y} B \rightarrow \mathbf{y} C$ is an epi in Sets $^{\mathbb{A}}$. Because colimits are computed component-wise in the functor category Sets ${ }^{\mathbb{A}}$, the function $(\mathbf{y} m)_{C}: \mathbb{A}(B, C) \rightarrow \mathbb{A}(C, C)$ is surjective. Hence, there exists $r \in \mathbb{A}(B, C)$ such that $(\mathbf{y} m)_{C}(r)=\operatorname{id}_{C} \in \mathbb{A}(C, C)$, that is, $r \circ m=\operatorname{id}_{C}$. Therefore, the arrow $m$ has a retraction $r$. $\square$ 
Therefore, an (Epi, SplitMono)-factorization $A \stackrel{e}{\rightarrow} C \stackrel{m}{\rightarrow} B$ in $\mathbb{A}$ induces an (Epi, Mono)factorization $\mathbf{y} B \stackrel{\mathrm{y} m}{\rightarrow} \mathbf{y} C \stackrel{\mathrm{y} e}{\longmapsto} \mathbf{y} A$. This yields

$$
\begin{aligned}
\{\operatorname{Im}(\mathbf{y} B \stackrel{\mathbf{y} f}{\rightarrow} \mathbf{y} A) \mid B \in \mathbb{A}, f: A \rightarrow B\} & =\{(\mathbf{y} C \stackrel{\mathrm{y} e}{\mapsto} \mathbf{y} A) \in \operatorname{Sub}(\mathbf{y} A) \mid C \in \mathbb{A}, e: A \rightarrow C\} \\
& \cong\{(A \stackrel{e}{\rightarrow} C) \in \operatorname{Quot}(A) \mid C \in \mathbb{A}\}=\operatorname{Quot}(A),
\end{aligned}
$$

where the last isomorphism holds because the functor $\mathbf{y}$ is full and faithful. Hence, the item 2 reduces to the item 1 .

\section{References}

Abramsky, S. and Winschel, V. (2015). Coalgebraic analysis of subgame-perfect equilibria in infinite games without discounting. Mathematical Structures in Computer Science. To appear.

Adámek, J. (2003). On final coalgebras of continuous functors. Theoretical Computer Science 294 $(1 / 2) 3-29$.

Adámek, J., Bonchi, F., Hülsbusch, M., König, B., Milius, S. and Silva, A. (2012). A coalgebraic perspective on minimization and determinization. In: Birkedal, L. (ed.) Proceedings of the Foundations of Software Science and Computational Structures - 15th International Conference, FoSSaCS 2012, Held as Part of the European Joint Conferences on Theory and Practice of Software, Lecture Notes in Computer Science, vol. 7213, Springer, 58-73.

Adámek, J. and Rosický, J. (1994). Locally Presentable and Accessible Categories, London Mathematical Society Lecture Note Series, vol. 189, Cambridge University Press.

Atkey, R., Ghani, N., Jacobs, B. and Johann, P. (2012). Fibrational induction meets effects. In: Birkedal, L. (ed.) Proceedings of the Foundations of Software Science and Computational Structures - 15th International Conference, FOSSACS 2012, Held as Part of the European Joint Conferences on Theory and Practice of Software, Lecture Notes in Computer Science, vol. 7213, Springer, $42-57$.

Bénabou, J. (1975). Théories relatives à un corpus. Comptes Rendus de l'Académie des Sciences Paris 281 A831-A834.

Bertot, Y. and Komendantskaya, E. (2008). Inductive and coinductive components of corecursive functions in Coq. Electronic Notes in Theoretical Computer Science 203 (5) 25-47.

Bonchi, F., Petrisan, D., Pous, D. and Rot, J. (2014). Coinduction up-to in a fibrational setting. In: Henzinger, T.A. and Miller, D. (eds.) Joint Meeting of the 23rd EACSL Annual Conference on Computer Science Logic (CSL) and the 29th Annual ACM/IEEE Symposium on Logic in Computer Science (LICS), CSL-LICS '14, ACM, 20.

Bonchi, F. and Pous, D. (2013). Checking NFA equivalence with bisimulations up to congruence. In Giacobazzi, R. and Cousot, R., (eds). The 40th Annual ACM SIGPLAN-SIGACT Symp osium on Principles of Programming Languages, POPL '13, ACM, 457-468.

Bradfield, J. and Stirling, C. (2006). Modal mu-calculi. In: Blackburn, P., van Benthem, J. and Wolter, F. (eds.) Handbook of Modal Logic, Studies in Logic and Practical Reasoning, vol. 3, chapter 12. Elsevier.

Cîrstea, C. (2011). Maximal traces and path-based coalgebraic temporal logics. Theoretical Computer Science 412 (38) 5025-5042.

Cîrstea, C., Kupke, C. and Pattinson, D. (2009). EXPTIME tableaux for the coalgebraic $\mu$-calculus. In: Grädel, E. and Kahle, R. (eds.) CSL, Lecture Notes in Computer Science, vol. 5771, Springer, 179-193. 
Cîrstea, C., Kurz, A., Pattinson, D., Schröder, L., and Venema, Y. (2011). Modal logics are coalgebraic. Comput. J. 54 (1) 31-41.

Cîrstea, C. and Sadrzadeh, M. (2008). Modular games for coalgebraic fixed point logics. Electronic Notes in Theoretical Computer Science 203 (5) 71-92.

Cousot, P. and Cousot, R. (1979). Constructive versions of Tarski's fixed point theorems. Pacific Journal of Mathematics 82 (1) 43-57.

Ferrari, G.L., Montanari, U. and Pistore, M. (2002). Minimizing transition systems for name passing calculi: A co-algebraic formulation. In: Nielsen, M. and Engberg, U. (eds.) Foundations of Software Science and Computation Structures, Proceedings of the 5th International Conference, FoSSaCS 2002. Held as Part of the Joint European Conferences on Theory and Practice of Software, Lecture Notes in Computer Science, vol. 2303, Springer, 129-158.

Ferrari, G.L., Montanari, U. and Tuosto, E. (2005). Coalgebraic minimization of HD-automata for the $\pi$-calculus using polymorphic types. Theoretical Computer Science 331 (2-3) 325-365.

Fiore, M. and Turi, D. (2001). Semantics of name and value passing. In: Logic in Computer Science, IEEE, Computer Science Press, 93-104.

Fiore, M.P. and Staton, S. (2006). Comparing operational models of name-passing process calculi. Information and Computer 204 (4) 524-560.

Fiore, M.P. and Staton, S. (2009). A congruence rule format for name-passing process calculi. Information and Computer 207 (2) 209-236.

Fumex, C., Ghani, N. and Johann, P. (2011). Indexed induction and coinduction, fibrationally. In: Corradini, A., Klin, B. and Cîrstea, C. (eds.) Proceedings of the Algebra and Coalgebra in Computer Science - 4th International Conference, Lecture Notes in Computer Science, vol. 6859, Springer, 176-191.

Gabriel, P. and Ulmer, F. (1971). Lokal Präsentierbare Kategorien. Springer.

Hasuo, I. (2010). Generic forward and backward simulations II: Probabilistic simulation. In: Gastin, P. and Laroussinie, F. (eds.) (2010). CONCUR 2010, Lecture Notes in Computer Science, vol. 6269, Springer, 447-461.

Hasuo, I., Cho, K., Kataoka, T. and Jacobs, B. (2013). Coinductive predicates and final sequences in a fibration. In: Mathematical Foundations of Programming Semantics (MFPS XXIX), Electronic Notes in Theoretical Computer Science, 197-214.

Hasuo, I., Jacobs, B. and Sokolova, A. (2007). Generic trace semantics via coinduction. Logical Methods in Computer Science 3 (4:11).

Hasuo, I., Shimizu, S. and Cîrstea, C. (2016). Lattice-theoretic progress measures and coalgebraic model checking. In: Bodik, R. and Majumdar, R. (eds.) Proceedings of the 43rd Annual ACM SIGPLAN-SIGACT Symposium on Principles of Programming Languages, ACM, 718-732.

Hennessy, M. and Milner, R. (1985). Algebraic laws for nondeterminism and concurrency. Journal of ACM 32 (1) 137-161.

Hensel, U. and Jacobs, B. (1997). Proof principles for datatypes with iterated recursion. In: Moggi, E. and Rosolini, G. (eds.) Category Theory and Computer Science, Lecture Notes in Computer Science, vol. 1290, Springer, Berlin, 220-241.

Hermida, C. (1993). Fibrations, Logical Predicates and Indeterminates. PhD thesis, Univ. Edinburgh. Techn. rep. LFCS-93-277.

Hermida, C. and Jacobs, B. (1998). Structural induction and coinduction in a fibrational setting. Information \& Computation 145 (2) 107-152.

Hur, C.-K., Neis, G., Dreyer, D. and Vafeiadis, V. (2013). The power of parameterization in coinductive proof. In: Giacobazzi, R. and Cousot, R. (eds.) (2013). The 40th Annual ACM SIGPLAN-SIGACT Symposium on Principles of Programming Languages, ACM, 193-206. 
Jacobs, B. (1999). Categorical Logic and Type Theory. North Holland, Amsterdam.

Jacobs, B. (2004). Trace semantics for coalgebras. In: Adámek, J. and Milius, S. (eds.) Coalgebraic Methods in Computer Science, Electronic Notes in Theoretical Computer Science, vol. 106, Elsevier, Amsterdam.

Jacobs, B. (2010). Predicate logic for functors and monads. Preprint, available at the author's webpage.

Jacobs, B. (2012). Introduction to coalgebra. Towards mathematics of states and observations. Draft of a book (ver. 2.0), available online.

Klin, B. (2007). Coalgebraic modal logic beyond Sets. In: MFPS XXIII, vol. 173, Elsevier, Amsterdam, 177-201.

Kozen, D. and Ruozzi, N. (2009). Applications of metric coinduction. Logical Methods in Computer Science 5 (3).

Kupke, C. (2007). Terminal sequence induction via games. In: Bosch, P., Gabelaia, D., and Lang, J. (eds.) TbiLLC, Lecture Notes in Computer Science, vol. 5422, Springer, 257-271.

Mac Lane, S. and Moerdijk, I. (1992). Sheaves in Geometry and Logic. A First Introduction to Topos Theory, Springer, New York.

Makkai, M. and Paré, R. (1989). Accessible categories: The foundations of categorical model theory. Contemporary Mathematics 104.

Miculan, M. (2008). A categorical model of the fusion calculus. Electronic Notes in Theoretical Computer Science 218 275-293.

Nakata, K., Uustalu, T. and Bezem, M. (2011). A proof pearl with the fan theorem and bar induction - walking through infinite trees with mixed induction and coinduction. In: Yang, $\mathrm{H}$. (eds.) APLAS, Lecture Notes in Computer Science, vol. 7078, Springer, 353-368.

Pattinson, D. (2003). An introduction to the theory of coalgebras. Course notes for NASSLLI. Available online.

Porst, H.-E. (2011). Algebraic lattices and locally finitely presentable categories. Algebra Universalis 65 (3) $285-298$.

Rutten, J.J.M.M. (2000). Universal coalgebra: A theory of systems. Theoretical Computer Science 249 (1) 3-80.

Stark, I. (1996). A fully abstract domain model for the $\pi$-calculus. In: Proceedings of the 11th Annual IEEE Symposium on Logic in Computer Science, IEEE Computer Society, 36-42.

Staton, S. (2011). Relating coalgebraic notions of bisimulation. Logical Methods in Computer Science 7 (1).

Venema, Y. (2006). Automata and fixed point logic: A coalgebraic perspective. Information and Computation 204 (4) 637-678.

Worrell, J. (2005). On the final sequence of a finitary set functor. Theoretical Computer Science 338 (1-3) 184-199. 